Aus der Abteilung Kognitive Neurologie

(Prof. Dr. rer. nat. M. Wilke)

im Zentrum Neurologische Medizin

der Medizinischen Fakultät der Universität Göttingen

\title{
Differenzierung motorischer kortiko-subkortikaler Netzwerke mit funktioneller Magnetresonanztomographie
}

\author{
INAUGURAL-DISSERTATION \\ zur Erlangung des Doktorgrades \\ der Medizinischen Fakultät \\ der Georg-August-Universität zu Göttingen \\ vorgelegt von \\ Julia Margarethe August geb. Böhm \\ aus Hannover
}

Göttingen 2012 
Dekan:

Prof. Dr. med. M.P. Schön

I. Berichterstatter: PD Dr. rer.nat. P. Dechent

II. Berichterstatterin: Prof. Dr. rer.nat. A. Antal

Tag der mündlichen Prüfung: 29.08.2012 


\section{Inhaltsverzeichnis}

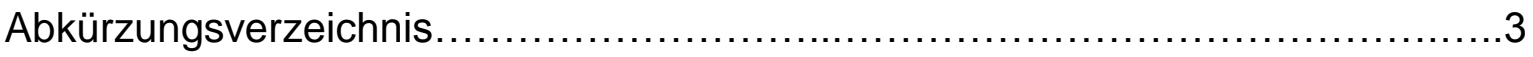

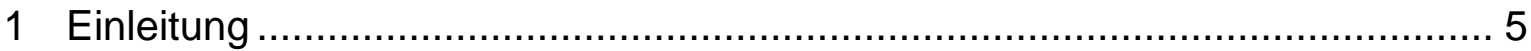

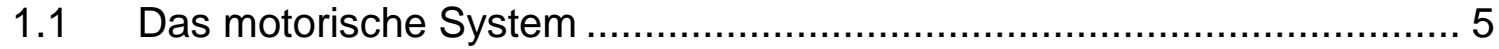

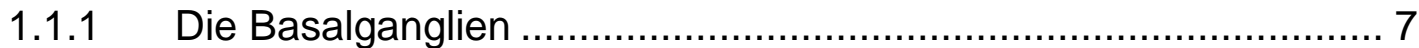

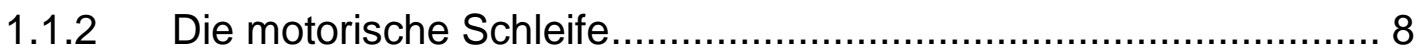

1.1.3 Parallele Schaltkreise innerhalb der motorischen Schleife ........... 11

1.1.4 Bildgebung von motorischen Schleifen ................................... 12

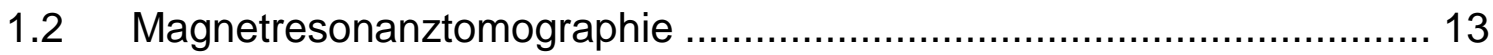

1.2.1 Physikalische Grundlagen .................................................... 14

1.2.2 Physiologische Grundlagen und Ablauf der fMRT ...................... 15

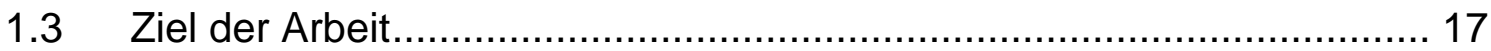

2 Probanden und Methoden......................................................................... 19

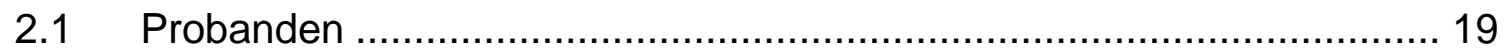

2.2 Motorische Aufgabe ................................................................. 19

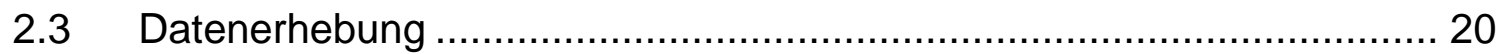

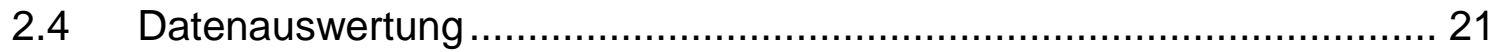

2.4.1 Auswertung der anatomischen Daten ..................................... 21

2.4.2 Auswertung der funktionellen Daten ....................................... 21

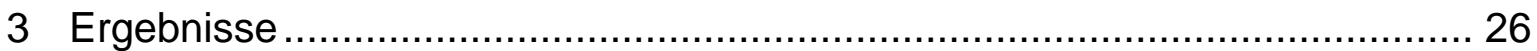

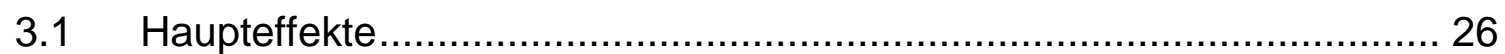

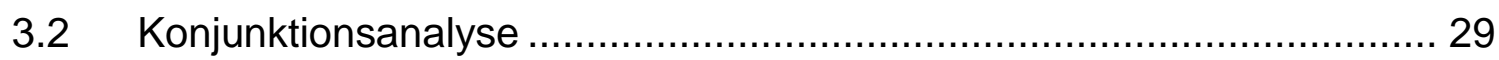

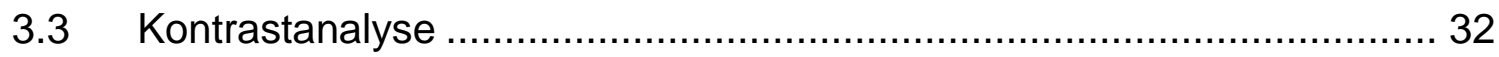

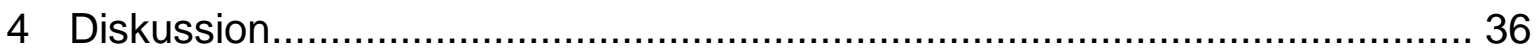

4.1 Das motorische Netzwerk und seine Lateralisierung ……................... 36

4.1.1 Das supplementär motorische Areal (SMA) ............................. 36

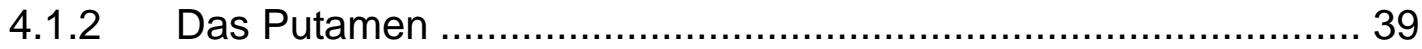

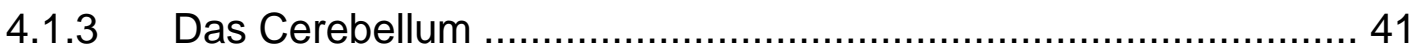

4.1.4 Koaktivierung und funktionelle Netzwerke................................ 42

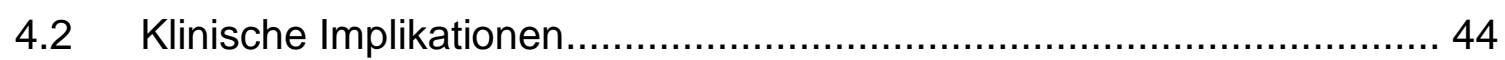

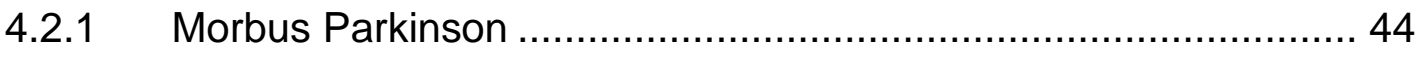




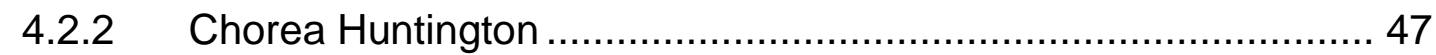

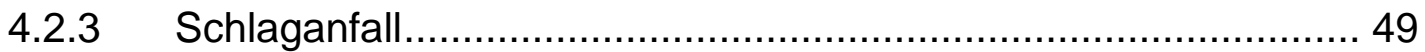

4.2.4 Tic-Störung und Tourette-Syndrom .......................................... 51

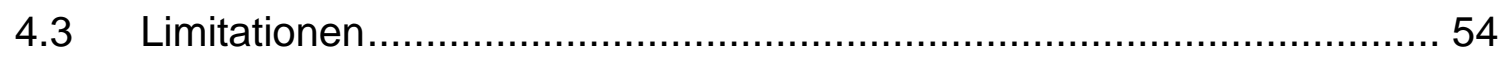

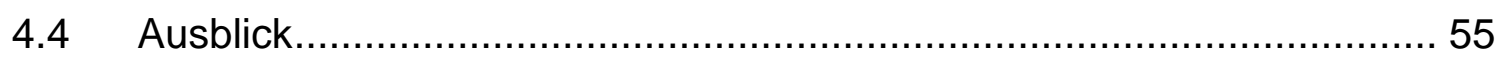

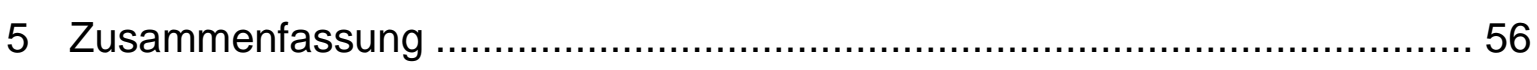

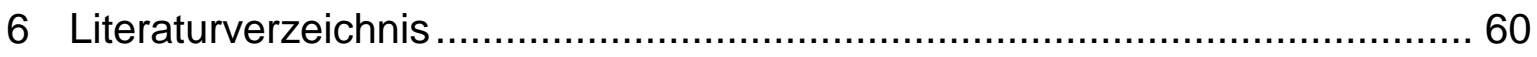

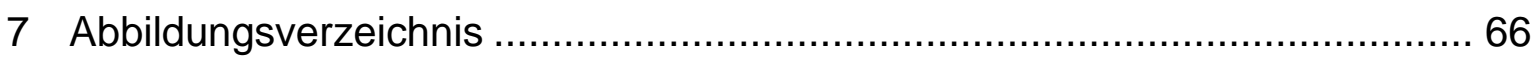

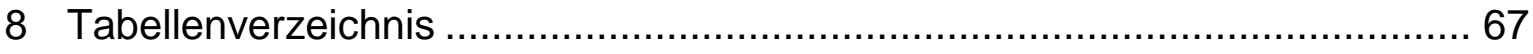




\section{Abkürzungsverzeichnis}

AC

ACC

$\mathrm{B}_{0}$

BA

BOLD-Effekt

CS

CST

CT

DLPFC

3D

EEG

EPI

FLASH

fMRT

FWHM

GLM

GP

$\mathrm{GPe}$

GPi

HD

HDRP

LCD

M1

MR

MRT

NC

NMR

PC

PD

PDRP
Anteriore Kommissur

Anteriorer Zingulärer Kortex

Vektor der Nettomagnetisierung

Brodmann-Areal

Blood Oxygenation Level Dependant-Effekt

Zentralsulkus

Cluster Size Thresholding (Methode zur Korrektur für multiple Vergleiche)

Computertomographie

Dorsolateraler Präfrontalkortex

Dreidimensional

Elektro-Enzephalographie

Echo Planar Imaging (echoplanare Bildgebung)

Fast Low Angle Shot

Funktionelle Magnetresonanztomographie

Full Width at Half Maximum (Halbwertsbreite)

General Linear Model (Allgemeines Lineares Modell)

Globus pallidus

Globus pallidus externus (äußerer Anteil)

Globus pallidus internus (innerer Anteil)

Huntington's Disease (Morbus Huntington)

Huntington-Disease Related Pattern

Liquid-Chrystal-Display

Primär motorischer Kortex

Magnetresonanz

Magnetresonanztomographie

Nucleus caudatus

Nuclear Magnetic Resonance

Posteriore Kommissur

Parkinson's Disease (Morbus Parkinson)

Parkinson-Disease Related Pattern 


\begin{tabular}{|c|c|}
\hline PET & Positronen-Emissions-Tomographie \\
\hline PM & Prämotorischer Kortex \\
\hline Prä-SMA & Prä-supplementär motorisches Areal \\
\hline Put. & Putamen \\
\hline SII & Sekundär somatosensorischer Kortex \\
\hline SMA & Supplementär motorisches Areal \\
\hline SMAC & Kaudaler Anteil des Supplementär motorischen Areals \\
\hline SMAr & Rostraler Anteil des Supplementär motorischen Areals \\
\hline SMC & Primär sensomotorischer Kortex \\
\hline SN & Substantia nigra \\
\hline SPECT & $\begin{array}{l}\text { Single Photon Emission Computed Tomography } \\
\text { (Einzelphotonen-Emissionscomputertomographie) }\end{array}$ \\
\hline STN & Nucleus Subthalamicus \\
\hline $\mathrm{T}_{1}$ & Wichtung der MRT-Messung (Spin-Gitter-Relaxation) \\
\hline $\mathrm{T}_{2}$ & Wichtung der MRT-Messung (Spin-Spin-Relaxation) \\
\hline $\mathrm{T}_{2}^{*}$ & Wichtung der MRT-Messung \\
\hline TE & Time of Echo (Echozeit) \\
\hline Thal. & Thalamus \\
\hline TR & Time of Repetition (Repetitionszeit) \\
\hline TS & Tourette-Syndrom \\
\hline VAC-line & eine zur anterioren Kommissur vertikale Linie \\
\hline VBM & $\begin{array}{l}\text { Voxel based morphometry (Analysemethode in der MR- } \\
\text { Forschung) }\end{array}$ \\
\hline VMAT2 & vesikulärer Monoamin-Transporter Typ 2 \\
\hline VPC-line & eine zur posterioren Kommissur vertikale Linie \\
\hline
\end{tabular}




\section{Einleitung}

Das motorische System des Menschen beschäftigt die Hirnforschung seit vielen Jahrzehnten. Die Willkürmotorik ermöglicht uns basale Fähigkeiten wie das Laufen und Greifen, aber auch anspruchsvolle Tätigkeiten wie Handwerk, Sport, Schreiben oder auch das Sprechen. Diesen uns im Alltag so selbstverständlich erscheinenden Bewegungsabläufen liegt ein komplexes neuronales Verschaltungssystem zugrunde, das aus verschiedenen Hirnstrukturen und ihren Verknüpfungen besteht. Ihre Kenntnis ist Voraussetzung für ein Verständnis dafür, wie vage Handlungsideen in konkrete motorische Planungen übergehen und schließlich in präzise Bewegungen mit wohl abgestimmter Aktivität verschiedener Muskelgruppen umgesetzt werden können.

Die Techniken zur Untersuchung des Gehirns und seiner Funktionen haben sich in den vergangenen Jahrzehnten rasant gewandelt. Bis zur Entdeckung und Etablierung der bildgebenden Verfahren erfolgte die Forschung überwiegend an post mortem entnommenen menschlichen Hirnen oder am Tiermodell. Es handelte sich um anatomische oder physiologische Studien, deren invasiver Charakter die Anwendung am lebenden Menschen verbot. Die Computertomographie (CT) und die Magnetresonanztomographie (MRT) boten erstmals die Möglichkeit, das lebende Gehirn nicht-invasiv dazustellen. Die funktionelle Magnetresonanztomographie (fMRT) schließlich ist, ähnlich wie die PositronenEmissions-Tomographie (PET), ein verhältnismäßig neues Verfahren, mit dessen Hilfe Gehirnfunktionen in vivo, also am wachen, denkenden, mitarbeitenden Probanden untersucht werden können.

\subsection{Das motorische System}

Der Entschluss zu einer Bewegung wird dem limbischen System zugeschrieben. Es setzt sich aus phylogenetisch alten Strukturen zusammen und besteht aus Hippokampus, Gyrus cinguli, Gyrus parahippocampalis, Corpus amygdaloideum sowie den Corpora mammillaria. Dieses funktionell zusammengehörende System spielt eine Rolle bei der Gedächtnisbildung, im emotionalen Erleben und Affektverhalten, bei der Modulation vegetativer Funktionen sowie für den psycho- und lokomotorischen Antrieb (Trepel 2004). Im nächsten Schritt gilt es, die angestrebte Handlung zu planen und einen Ablauf festzulegen. Für die Vorbereitung und Planung einer Bewegung hat der präfrontale 
Assoziationskortex eine entscheidende Bedeutung. Dieser befindet sich im Bereich des Brodmann-Areals (BA) 6. Den lateralen Anteil bildet der prämotorische Kortex (PM), hier werden Bewegungsentwürfe erstellt. Der mediale Anteil des BA 6 wird als supplementär motorisches Areal (SMA) bezeichnet, dieses spielt bei der konkreten Vorbereitung einer Handlung und der zeitlichen Abfolge eine Rolle.

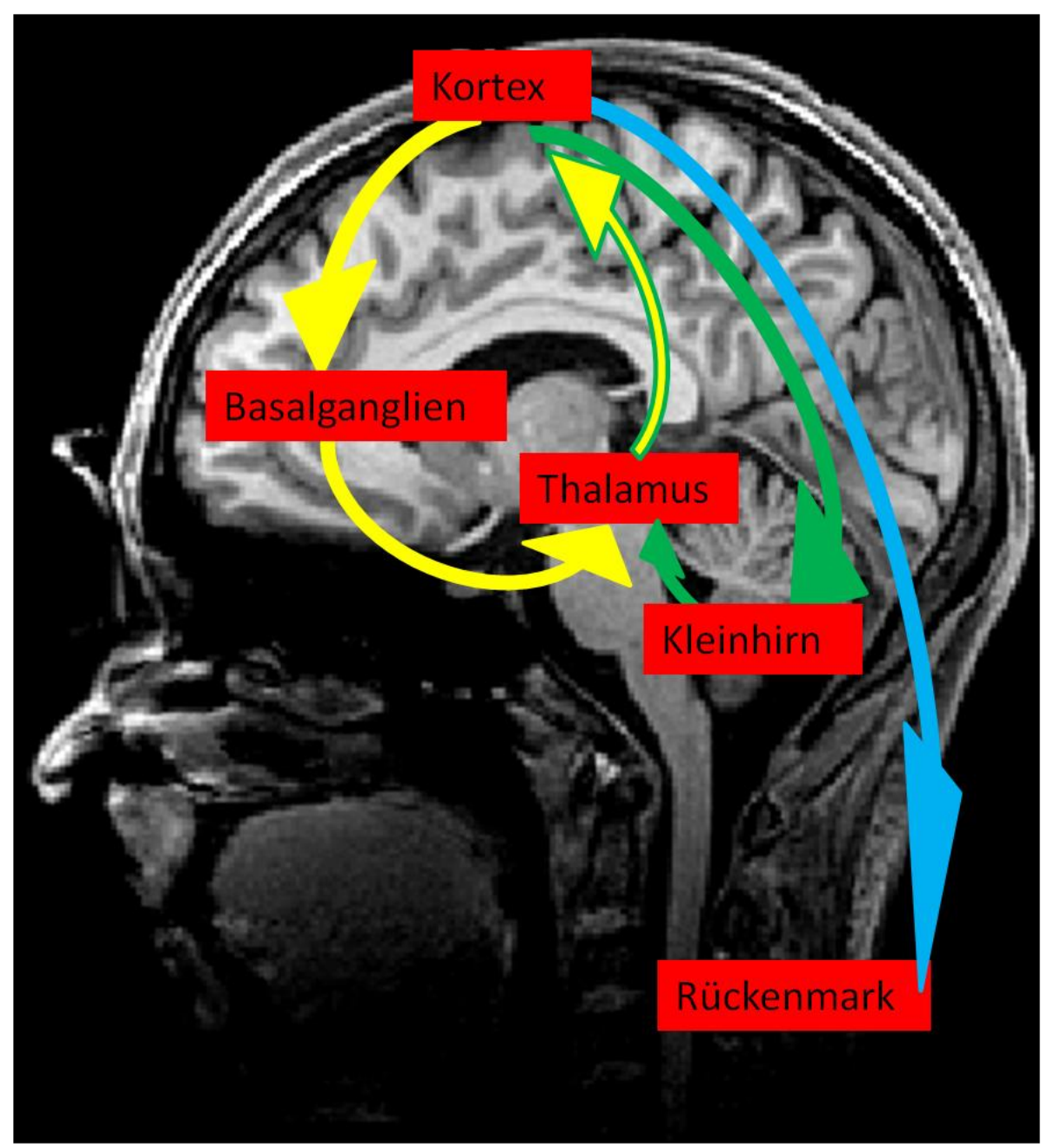

Abbildung 1: Die zentrale Regulation der Motorik. Bevor ein Handlungsbefehl vom Kortex über die Pyramidenbahn ins Rückenmark projiziert wird (blauer Pfeil), durchläuft der Handlungsentwurf zwei modulierende kortiko-subkortikale Schleifen: über die Basalganglien einerseits (gelbe Pfeile) und über das Kleinhirn andererseits (grüne Pfeile). Stark vereinfachtes Schema nach De Long 2000 und Trepel 2004.

Der letztliche Bewegungsbefehl geht vom primär motorischen Kortex (M1) aus. Es handelt sich hierbei um den präzentralen Gyrus, das BA 4. Hier nimmt der Tractus corticospinalis, die Pyramidenbahn, seinen Ursprung. Vorher sind jedoch eine Überarbeitung und eine 
Korrektur des Bewegungsentwurfes notwendig. Dies erfolgt im Wesentlichen in zwei kortiko-subkortikalen Schleifen, wovon eine über die Basalganglien, die andere über das Kleinhirn verläuft (s. Abbildung 1). Im Kleinhirn erfolgt die Feinabstimmung für eine flüssige, koordinierte Bewegung. Die modifizierten Informationen aus Basalganglien und Kleinhirn gelangen in den Thalamus, von wo sie nach einer Integration zurück in die kortikalen motorischen Areale projiziert werden (DeLong 2000; Trepel 2004).

Insbesondere die erstgenannte Informationsschleife über die Basalganglien soll Thema dieser Arbeit sein und genauer untersucht werden.

\subsubsection{Die Basalganglien}

Die Basalganglien bestehen aus vier Kernen, dem Striatum, dem Globus pallidus (GP), der Substantia nigra (SN) und dem Nucleus subthalamicus (STN). Das Striatum kann seinerseits wiederum in Nucleus caudatus (NC) und Putamen unterteilt werden, der Globus pallidus in einen externen (GPe) und einen internen (GPi) Anteil. Entwicklungsgeschichtlich haben die Kerne einen gemeinsamen Ursprung, und sie besitzen enge reziproke Verknüpfungen (DeLong 2000). Ihre Aufgabe besteht in einer Modulation des Bewegungsentwurfes, insbesondere hinsichtlich Bewegungsausmaß und Kraftdosierung. Der Morbus Parkinson sowie die Chorea Huntington sind gut erforschte Erkrankungen, bei denen ein zu starker bzw. zu schwacher Einfluss der Basalganglien für die Symptome ursächlich ist.

Das Verschaltungsprinzip der Basalganglien ist außerordentlich komplex (s. Abbildung 2). Die Efferenzen aus dem Kortex gelangen zunächst ins Striatum. Nach einer ersten Modulation wird die Bewegungsinformation anschließend in die Substantia nigra einerseits, in den Globus pallidus andererseits und von hier aus in den Nucleus subthalamicus weitergeleitet, um schließlich im Globus pallidus wieder integriert zu werden. Die einzige efferente Struktur der Basalganglien ist der Globus pallidus internus. Hier laufen die modulierten Informationen zusammen und gelangen zum Thalamus. Im Thalamus werden die Schleifen aus den Basalganglien einerseits und aus dem Kleinhirn andererseits integriert. Hier entspringen Verknüpfungen zurück zum Kortex sowie eine direkte Verbindung zum Rückenmark. 


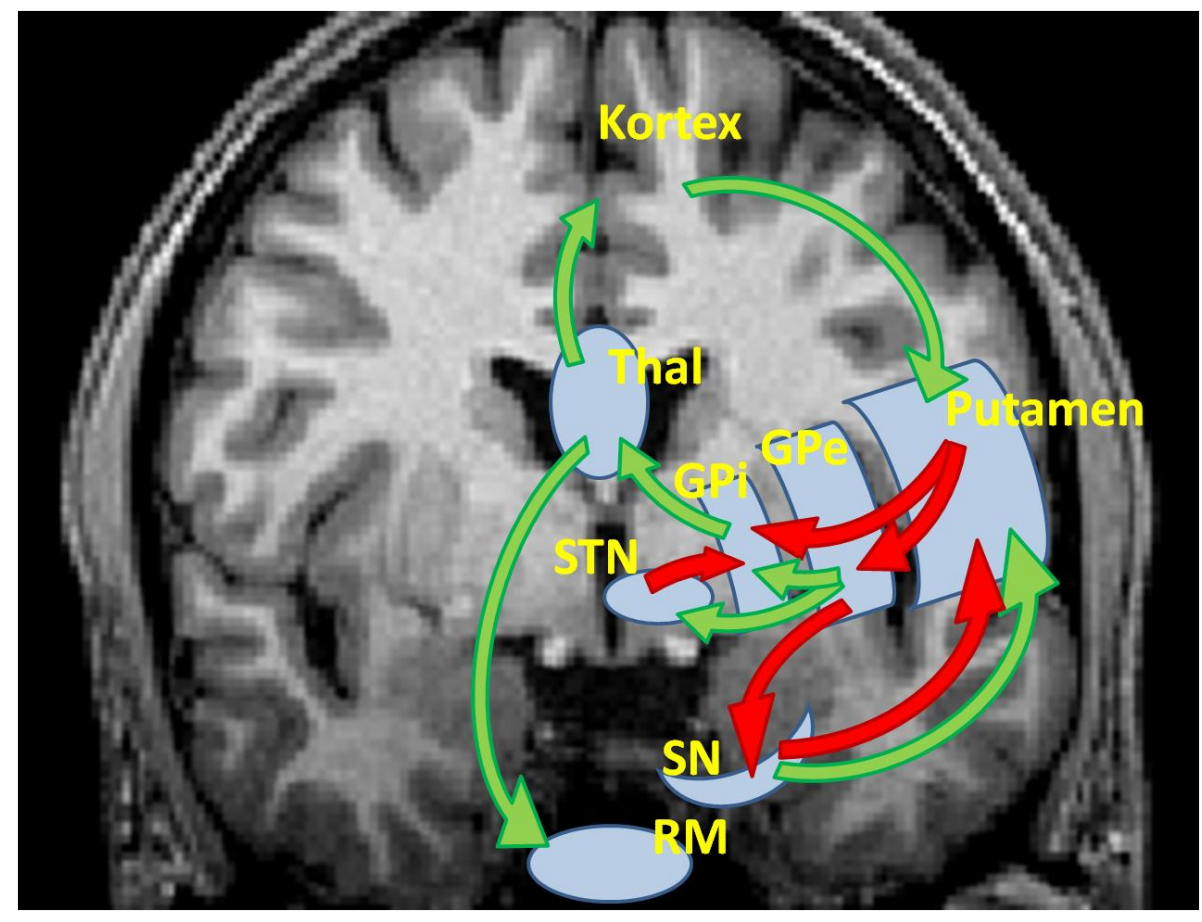

Abbildung 2: Die Verschaltung der Basalganglien. Die kortikalen Efferenzen gelangen ins Putamen. Über multiple teils exzitatorische (grüne Pfeile), teils inhibitorische (rote Pfeile) neuronale Verknüpfungen erfolgt eine komplexe Modulation des Bewegungsentwurfes in Arealen des GPe, des GPi, des STN sowie der SN. Die einzige Efferenz der Basalganglien entspringt dem GPi. Nach Integration im Thalamus (Thal) gelangt die Bewegungsinformation wieder zum Kortex sowie teilweise direkt ins Rückenmark (RM). Vereinfacht und modifiziert nach DeLong 2000.

\subsubsection{Die motorische Schleife}

Neuronale Verbindungen zwischen Kortex, Basalganglien und Thalamus sind schleifenförmig organisiert. Sowohl im Tiermodell als auch am Menschen, in vitro und in vivo, wurden ihre anatomischen und funktionellen Eigenschaften erforscht. Während man anfangs glaubte, die Basalganglien hätten die Funktion, Informationen unterschiedlicher Kortexanteile zu bündeln und zu kanalisieren (Kemp und Powell 1971), hat sich in den letzten Jahrzehnten eine neue Auffassung durchgesetzt. Man fand nämlich, dass keine Bündelung, sondern eher eine „Parallelschaltung“ vorliegt, die Informationen verschiedener Kortexareale nicht etwa zusammenfasst, sondern unabhängig voneinander prozessiert. 
A

\begin{tabular}{|c|c|c|c|c|}
\hline Motorisch & Okulomotorisch & Präfrontal & Orbitofronal & Zingulär \\
\hline SMA & Frontales Augenfeld & $\begin{array}{l}\text { Dorsola } \\
\text { Präfronte }\end{array}$ & $\begin{array}{c}\text { Lateraler } \\
\text { Orbitrontalkortex }\end{array}$ & ACC \\
\hline$\downarrow$ & $\downarrow$ & $\downarrow$ & $\downarrow$ & $\downarrow$ \\
\hline Putamen & Nucleus caudatus & $\begin{array}{l}\text { Dorsolateraler } \\
\text { Nucleus caudatus }\end{array}$ & $\begin{array}{l}\text { Ventromedialer } \\
\text { Nucleus caudatus }\end{array}$ & Ventrales Striatum \\
\hline$\stackrel{\downarrow}{\downarrow} \mathrm{GPi}^{\mathrm{S} N}$ & $\stackrel{\downarrow}{\downarrow}$ & $\stackrel{\downarrow}{\downarrow}$ & $\stackrel{\downarrow}{\downarrow} \mathrm{GPi}^{\mathrm{S} N}$ & $\stackrel{\downarrow}{\downarrow} \mathrm{GPi}^{\mathrm{SSN}}$ \\
\hline$\downarrow$ & $\downarrow$ & $\downarrow$ & $\downarrow$ & $\downarrow$ \\
\hline $\begin{array}{c}\text { Ventroanteriorer } \\
\text { und dorsomedialer } \\
\text { Thalamus }\end{array}$ & $\begin{array}{c}\text { Ventroanteriorer } \\
\text { und dorsomedialer } \\
\text { Thalamus }\end{array}$ & $\begin{array}{c}\text { Ventroanteriorer } \\
\text { und dorsomedialer } \\
\text { Thalamus }\end{array}$ & $\begin{array}{l}\text { Ventroanteriorer und } \\
\text { dorsomedialer } \\
\text { Thalamus }\end{array}$ & $\begin{array}{c}\text { Ventroanteriorer } \\
\text { und dorsomedialer } \\
\text { Thalamus }\end{array}$ \\
\hline
\end{tabular}

B

\begin{tabular}{|cccc|}
\hline Motorisch & Räumlich & Visuell & Affektiv \\
& Dorsolateraler & Ventrolateraler & Orbitofrontaler Kortex \\
SMA & Präfrontalkortex & Präfrontalkortex & $\downarrow$ \\
$\downarrow$ & $\downarrow$ & $\downarrow$ & $\downarrow$ \\
Putamen & Caput nuclei caudati & Cauda nuclei caudati & Ventrales Striatum \\
$\downarrow$ & $\downarrow$ & ventralis & $\downarrow$ \\
$\mathrm{GPi} / \mathrm{SN}$ & $\mathrm{GPi} / \mathrm{SN}$ & $\mathrm{GPi} / \mathrm{SN}$ & Ventraler GP \\
$\downarrow$ & $\downarrow$ & $\downarrow$ & $\downarrow$ \\
Ventroanteriorer und & Ventroanteriorer und & Ventroanteriorer und & Dorsomedialer Thalamus \\
ventrolateraler Thalamus & dorsomedialer Thalamus & dorsomedialer Thalamus & \\
& & & \\
\hline
\end{tabular}

C

\begin{tabular}{|c|c|c|}
\hline Motorisch & Assoziativ & Limbisch \\
\hline $\begin{array}{l}\text { Motorischer, prämotorischer, } \\
\text { supplementär-motorischer, } \\
\text { zingulärer und somatosensorischer } \\
\text { Kortex }\end{array}$ & $\begin{array}{l}\text { Präfrontaler, temporaler, posterior } \\
\text { parietaler, präokzipitaler Kortex, } \\
\text { frontales Augenfeld und supple- } \\
\text { mentäres Augenfeld }\end{array}$ & $\begin{array}{l}\text { Hippokampus; piriformer, } \\
\text { prälimbischer und infralimbischer } \\
\text { Kortex, Amygdala }\end{array}$ \\
\hline$\downarrow$ & $\downarrow$ & $\downarrow$ \\
\hline $\begin{array}{l}\text { Postkommissurales Putamen, } \\
\text { dorsolaterale Anteile }\end{array}$ & $\begin{array}{l}\text { Nucleus caudatus, } \\
\text { Präkommissurales Putamen }\end{array}$ & $\begin{array}{l}\text { Ventrale Anteile des Nucleus } \\
\text { caudatus, ventrales Putamen }\end{array}$ \\
\hline$\downarrow$ & $\downarrow$ & $\downarrow$ \\
\hline $\begin{array}{l}\text { Ventrolaterale Anteile des } \\
\text { kaudalen GPi }\end{array}$ & Ventrales/mediales GPi & Ventrales/rostrales GP \\
\hline$\downarrow$ & & $\downarrow$ \\
\hline Ventrolateraler Thalamus & $\begin{array}{c}\text { Ventroanteriorer/ } \\
\text { ventrolateraler Thalamus }\end{array}$ & Ventroanteriorer Thalamus \\
\hline
\end{tabular}

Abbildung 3: Verschiedene Modelle kortiko-basalganglien-thalamo-kortikaler Schleifen, vereinfacht und modifiziert. A) Alexander et al. erstellten erstmals 1986 ein Modell kortikostriataler Schleifen (nach Alexander et al. 1986, Seite 364. B) Modifikation nach Lawrence et al. 1998, Seite 380. C) Modifikation nach Nakano et al. 2000.

ACC: Anteriorer zingulärer Kortex. 
Ein zuerst von Alexander et al. (1986) vorgeschlagenes Modell beschreibt hierbei mehrere parallele Schaltkreise (s. Abbildung 3, A). Innerhalb dieser Kreise wird eine anatomisch und funktionell getrennte Verknüpfung von einzelnen Anteilen beteiligter Hirnstrukturen postuliert. Dieses Modell wurde vielfach diskutiert und modifiziert, beispielsweise von Lawrence et al. (1998) (s. Abbildung 3 B). Breite Anerkennung kommt heute einem Modell zu, nach dem Kortex und Striatum und demnach kortiko-striatale Projektionen in drei sowohl anatomisch als auch funktionell getrennte Schleifen unterteilt werden können: die sensomotorische, die assoziative (von manchen Autoren auch als prämotorische Schleife bezeichnet) und die limbische Schleife (s. Abbildung 3 C). Ihre Anteile übermitteln Informationen zwischen den jeweiligen kortikalen und subkortikalen Arealen (Selemon und Goldman-Rakic 1985; Alexander und Crutcher 1990; Parent 1990; Parent und Hazrati 1995; Nakano et al. 2000; Postuma und Dagher 2006; Di Martino et al. 2008).

In dem heute anerkannten Modell (s. Abbildung $3 \mathrm{C}$ ) besteht das dorsale Striatum (Putamen und Nucleus caudatus, NC) demnach aus zwei Kompartimenten: dem sensomotorischen einerseits und dem assoziativen andererseits, jeweils eingebettet in die entsprechenden Schaltkreise (Kunzle 1975; Selemon und Goldman-Rakic 1985; Parent und Hazrati 1995; Nakano et al. 2000). Die Aufteilung scheint sich entlang einer rostrokaudalen / mediolateralen Achse zu definieren. Kaudolaterale Anteile des Putamens (posterior der anterioren Kommissur, AC) erhalten Afferenzen aus kortikalen motorischen Arealen und können daher als sensomotorisches Kompartiment gelten (Postuma und Dagher 2006). Rostromediale Anteile (anterior der AC) und der Kopf des NC repräsentieren das assoziative Kompartiment, sie sind mit präfrontalen kortikalen Arealen verknüpft (Kunzle 1975; Parent 1990; Parent und Hazrati 1995). Das ventrale Striatum ist Teil der dritten, limbischen Schleife, diese wird jedoch in dieser Arbeit wegen des Schwerpunkts auf motorische Aspekte nicht diskutiert. Auch innerhalb der beschriebenen Schleifen existieren sog. „subloops“ (=untergeordnete Schleifen), in denen spezielle Kortexareale mit subkortikalen strukturen schleifenförmig verknüpft sind (Nakano et al. 2000). 


\subsubsection{Parallele Schaltkreise innerhalb der motorischen Schleife}

Betrachtet man die einzelnen kortikalen Areale und ihre Projektionen, so sind der primär sensomotorische Kortex (SMC) und das SMA mit dem sensomotorischen Kompartiment des Putamens verknüpft, sie bilden entsprechend einen Teil der sensomotorischen Schleife (Lehericy et al. 2004b). Prä-SMA und prämotorische kortikale Regionen hingegen projizieren ins rostromediale, assoziative Kompartiment des Putamens (Lehericy et al. 2004b; Leh et al. 2007). Die Grenze zwischen SMA und Prä-SMA ist dabei etwa auf dem Niveau einer vertikalen Linie von der AC zu lokalisieren (Picard und Strick 1996; Zilles et al. 1996).

Die kortikostriatalen Projektionen der verschiedenen Schleifen unterscheiden sich u.a. in Bezug auf die Somatotopie. So ist sowohl vom SMC als auch vom SMA eine strenge Somatotopie gut bekannt, das Prä-SMA hingegen ist nicht somatotopisch organisiert (Grafton et al. 1996; Picard und Strick 1996; Picard und Strick 2001; Lehericy et al. 2004a). Innerhalb des dorsalen Striatums sind motorische Afferenzen entlang einer ventrodorsalen Achse somatotopisch angeordnet: hierbei liegt das Areal für Afferenzen aus dem Gesichtsbereich ventral, aus der unteren Extremität dorsal, und aus der oberen Extremität mittig (Kunzle 1975; Flaherty und Graybiel 1993; Gerardin et al. 2003).

Ein anderes Unterscheidungsmerkmal der kortikostriatalen Projektionen ist das der Lateralität. Es konnte gezeigt werden, dass der menschliche SMC hauptsächlich ins ipsilaterale Striatum projiziert, während das SMA bilaterale Verknüpfungen ins Striatum hat - dennoch bleibt der Großteil der Projektionen ipsilateral (Wiesendanger et al. 1996; Gerardin et al. 2003). Demgegenüber weist das Prä-SMA bilaterale Projektionen zum Striatum auf, sowie mehr transkallosale Verbindungen (Lehericy et al. 2004a). Im Striatum selbst enden bilateral organisierte Afferenzen aus dem Kortex anterior von denjenigen, die unilateral organisiert sind (Kunzle 1975; Gerardin et al. 2003; Lehericy et al. 2004a). In der Zusammenschau mit dem Wissen über die Somatotopie kommt man zu der Annahme, dass die sensomotorische Schleife hauptsächlich eine unilaterale, die assoziative Schleife hingegen eine bilaterale Organisation aufweist. 


\subsubsection{Bildgebung von motorischen Schleifen}

In-vivo-Bildgebung der kortikostriatalen Schleifen am Menschen ist mittels nicht-invasiver Techniken wie PET oder fMRT möglich. Die visuelle Darstellung der Schleifen war bereits Gegenstand einiger Studien. Um die sensomotorische und die assoziative Schleife zu untersuchen, wurden verschiedene Eigenschaften einer motorischen Aufgabe (Bewegungsamplitude, -frequenz, -komplexität) unabhängig voneinander variiert (Sadato et al. 1996a; Sadato et al. 1996b; Boecker et al. 1998; Deiber et al. 1999; Lehericy et al. 2006). Ziel war es, die Schleifen spezifisch $\mathrm{zu}$ beeinflussen. Tatsächlich wurden Aktivitäten in sensomotorischen Arealen (z.B. SMC, SMA, nicht aber in der assoziativen Prä-SMA) von Eigenschaften wie Bewegungsamplitude und -frequenz verändert. Hingegen zeigten Komplexität der Aufgabe, Selbst-Initiierung der Bewegung oder eigene Rhythmusvorgabe einer Bewegungsabfolge eine Beeinflussung von assoziativen Arealen der Bewegungsplanung (Boecker et al. 1998; Jueptner und Weiller 1998; Lehericy et al. 2006). Wenngleich diese Studien den Kenntnisstand erheblich erweiterten, sind Gegenüberstellung und Unterscheidung der kortikostriatalen Bahnen und Schleifen nur indirekt über den Vergleich verschiedener motorischer Aufgaben möglich. Auch sind die berichteten Differenzen der Signalaktivitäten extrem gering, insbesondere in anatomisch kleinen Strukturen wie den Basalganglien. Schließlich beschränkt die Mehrzahl der Studien ihre Untersuchungen auf motorische Aufgaben der rechten Hand und somit der dominanten Hemisphäre (Jenkins et al. 1997; Boecker et al. 1998; Lehericy et al. 2006), sowie auf entweder den Kortex oder die Basalganglien, sie erfassen jedoch nicht die Gesamtheit der den Schleifen zugehörigen Strukturen (Boecker et al. 1998; Jueptner und Weiller 1998; Lehericy et al. 2006; Boecker et al. 2008).

In einer Metaanalyse haben Postuma und Dagher (2006) 126 funktionelle PET- und fMRTStudien auf eine Beteiligung der Basalganglien hin untersucht. Indem sie Muster von gemeinsamer Aktivierung verschiedener Hirnregionen identifizierten, konnten sie die Zusammensetzung der kortikostriatalen Schleifen ableiten und somit das zuvor erwähnte Modell der Dreiteiligkeit von Nakano et al. (2000) untermauern. Die Autoren betonen, dass sich die meisten Studien auf die Untersuchung der dominanten rechten Hand und der kontralateralen Hirnhälfte limitieren, und zeigen die Notwendigkeit weiterer Forschung insbesondere über die nicht-dominante Hand oder bilaterale Bewegungen auf. 


\subsection{Magnetresonanztomographie}

Die MRT wurde in den 1970er Jahren maßgeblich von Paul C. Lauterbur und Sir Peter Mansfield entwickelt (Lauterbur 1973). Sie hat seitdem ihren Siegeszug zunächst in der Forschung, später auch in der klinischen Anwendung angetreten. Das Verfahren hat eine hervorragende räumliche Auflösung, ist nicht-invasiv und kommt ohne ionisierende Strahlung aus. Innerhalb kurzer Zeit ist die MRT daher zu einem der wichtigsten Instrumente der bildgebenden Diagnostik einerseits und der Hirnforschung andererseits geworden. Ihr Entwickler Paul C. Lauterbur wurde für seine Forschung 2003 mit dem MedizinNobelpreis ausgezeichnet.

Ein weiterer Meilenstein in der Geschichte der MRT wurde durch den Göttinger Physiker Jens Frahm und Kollegen ermöglicht. Durch die Entwicklung der fast-low-angle-shotTechnik (FLASH-Technik) in den 1980er Jahren konnte die Messzeit für MRTAufnahmen um ein Vielfaches reduziert werden (Frahm et al. 1986a; Frahm et al. 1986b). Neben praktischen Gesichtspunkten für die raschere Anwendung im klinischen Alltag wurde die Bildgebung von dynamischen Prozessen durch die neue Technik erst möglich.

Die Entwicklung der fMRT anfang der 1990er Jahre fußte auf zwei grundlegenden Beobachtungen. Zum einen war bereits 1935 entdeckt worden, dass der Oxygenierungszustand von Hämoglobin dessen Eigenschaften im Magnetfeld beeinflusst (Pauling 1935). Zum anderen konnten Fox und Raichle 1986 zeigen, dass neuronale Aktivität mit einer lokalen Veränderung des Sauerstoffgehalts im umgebenden Gewebe einhergeht (Fox und Raichle 1986). Auch konnten unterschiedliche Oxygenierungszustände von Hämoglobin mittels MRT in vitro (Thulborn et al. 1982) dargestellt werden. Ogawa und Kollegen konnten 1990 diese Erkenntnisse kombinieren und erstmals in vivo Veränderungen des Oxygenierungszustandes von Hämoglobin mittels MRT detektieren (Ogawa et al. 1990). Von ihnen wurde der Begriff des BOLD-Signals (blood oxygenation level dependant signal) geprägt. Der Grundstein für die fMRT war gelegt. 


\subsubsection{Physikalische Grundlagen}

Die physikalischen Grundlagen gelten für die anatomische ebenso wie für die funktionelle MRT. Die Grundlage ist ein starkes statisches Magnetfeld. Übliche Feldstärken sind derzeit 1,5 bis 3 Tesla, letzteres entspricht etwa dem 60000 fachen der Erdanziehungskraft. Die MRT macht sich die magnetischen Eigenschaften von Wasserstoffprotonen zunutze, die etwa $80 \%$ aller Atome im menschlichen Körper ausmachen.

Protonen besitzen zwei Eigenschaften, die zu ihrer großen Bedeutung in der MRBildgebung führen. Einerseits sind Protonen aufgrund ihrer ungeraden Ladungszahl magnetische Dipole, andererseits zeigen sie eine Rotation um die eigene Achse (Spin). Ohne umgebendes Magnetfeld sind die einzelnen Protonen zufällig im Raum ausgerichtet. In einem statischen Magnetfeld jedoch führt der Spin zur Entstehung eines elektrischen Ringstroms und damit zu einem magnetischen Moment. Die Protonen richten sich entlang dem magnetischen Feld aus. Hierbei nimmt die Mehrzahl der Protonen eine parallele und damit niedrigenergetische Position ein, eine kleinere Anzahl hingegen eine antiparallele und damit höher energetische Position. Es resultiert eine Nettomagnetisierung $\mathbf{M}_{0}$ in Richtung des Magnetfeldes.

Wird mittels eines Hochfrequenzimpulses ein zum statischen Magnetfeld transversal stehendes Magnetfeld appliziert, so werden die Protonen in Richtung des neuen Magnetfeldes ausgelenkt. Dies wird an der Abnahme der longitudinalen und dem Aufbau einer transversalen Nettomagnetisierung erkennbar. Wird das zusätzliche Magnetfeld nun abgeschaltet, kommt es zur Wiederausrichtung der Protonen entlang des statischen Magnetfeldes, die ursprüngliche Nettomagnetisierung stellt sich wieder her. Die Zeitspanne, bis $63 \%$ der longitudinalen Nettomagnetisierung wieder erreicht sind, wird definitionsgemäß als ,longitudinale Relaxationszeit" oder auch $\mathrm{T}_{1}$-Zeit bezeichnet. Umgekehrt beschreibt die $\mathrm{T}_{2^{-}}$ Relaxationszeit die Zeitspanne, nach der $63 \%$ der transversalen Nettomagnetisierung zerfallen sind. Beide Relaxationszeiten unterscheiden sich für verschiedene Gewebearten und Flüssigkeiten, wodurch eine Differenzierung im $\mathrm{T}_{1}$ - bzw. $\mathrm{T}_{2}$-gewichteten MRT-Bild möglich wird. Für die BOLD-fMRT wird die T2*-Wichtung genutzt, die sensibel für lokale Magnetfeld-Inhomogenitäten ist, z.B. verursacht durch den Eisengehalt der untersuchten Substanz. Damit eignet sie sich hervorragend für die Darstellung der Einflüsse von Hämoglobin in unterschiedlichen Oxygenierungszuständen. 


\subsubsection{Physiologische Grundlagen und Ablauf der fMRT}

Werden bei kognitiven Aufgaben Kortexareale aktiviert, so kommt es zunächst zu einem vermehrten Verbrauch von Sauerstoff in diesen Arealen und somit zum Anstieg von desoxygeniertem Hämoglobin (s. Abbildung 4). Kompensatorisch kommt es zu einer lokalen Steigerung des Blutflusses, die Konzentration von oxygeniertem Hämoglobin steigt an. Diese sogenannte „Neurovaskuläre Kopplung“ macht sich die fMRT zunutze: aus Veränderungen des lokalen Blutflusses wird auf Veränderungen der neuronalen Aktivität einer Hirnregion geschlossen, und damit auf das Beteiligtsein dieser Hirnregion an der untersuchten kognitiven Aufgabe (Arthurs und Boniface 2002). Es handelt sich somit um eine indirekte Methode der Messung neuronaler Aktivität.

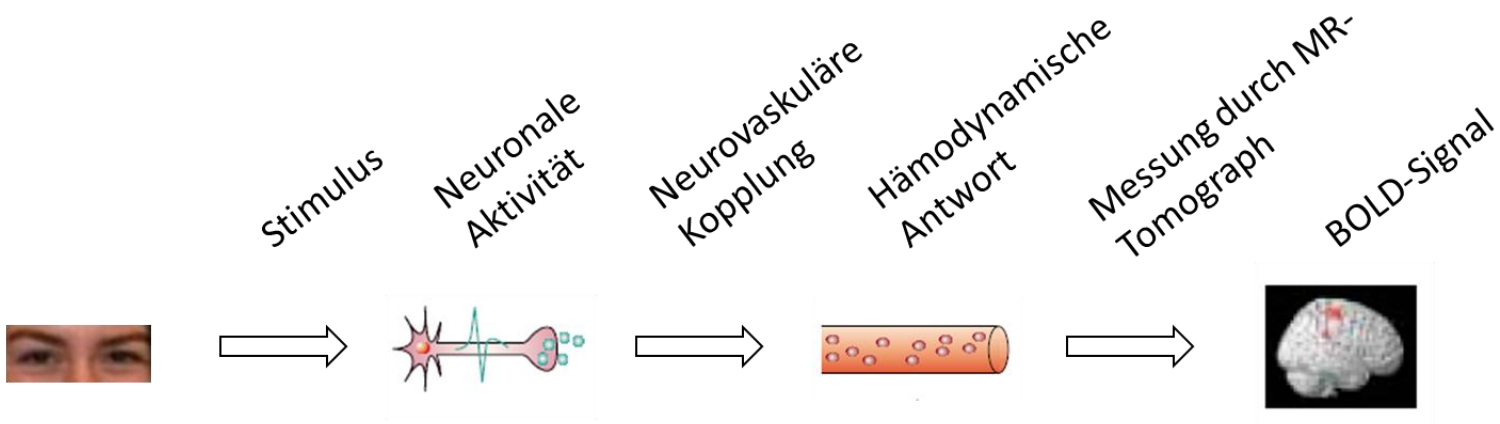

Abbildung 4: Die Entstehung des BOLD-Signals. Auf einen Stimulus erfolgt eine neuronale Antwort. Über einen komplexen Prozess, die sog. Neurovaskuläre Kopplung, wird eine hämodynamische Reaktion hervorgerufen. Die Schwankungen des Desoxyhämoglobingehalts im Blut werden durch den Magnetresonanztomographen detektiert und über Rechenprozeduren sichtbar gemacht (modifiziert und übersetzt nach Arthurs und Boniface 2002, Seite 28).

Das BOLD-Signal zeigt einen charakteristischen zeitlichen Ablauf, die „Hämodynamische Antwort" (s. Abbildung 5). Während die neuronale Aktivierung auf einen externen (beispielsweise visuellen) Stimulus innerhalb von Millisekunden erfolgt und z.B. mittels der Elektroenzephalographie (EEG) gemessen werden kann, verläuft die hämodynamische Antwort verzögert. Es kommt zunächst zum initialen Abfall, anschließend zur für die Auswertung relevanten positiven BOLD-Antwort sowie schließlich zur Post-StimulusUnterschreitung. Die lokale Signalveränderung, die als BOLD-Antwort im fMRT- 
Experiment gemessen wird, beträgt je nach Studiendesign und untersuchtem Areal etwa 0,1-5\% (Goebel und Kriegeskorte 2005).

Für neurowissenschaftliche Fragestellungen liegt der Proband auf dem Rücken auf einer Untersuchungsliege, die vor Beginn der Messung ins Zentrum des Magnetfeldes bewegt wird. Der Kopf des Probanden befindet sich in einer Kopfspule, für Lärmschutz und Kommunikation werden Kopfhörer, zur Stimulation eine magnettaugliche Liquid-ChrystalDisplay (LCD)-Brille getragen. Der MR-Tomograph selbst sowie Kopfhörer und Brille können über eine Bedienungskonsole von einem abgeschirmten Nebenraum aus angesteuert werden. Ein Computer dient zur Darbietung des Stimulationsprotokolls und zum Empfang der Verhaltensdaten des Probanden.

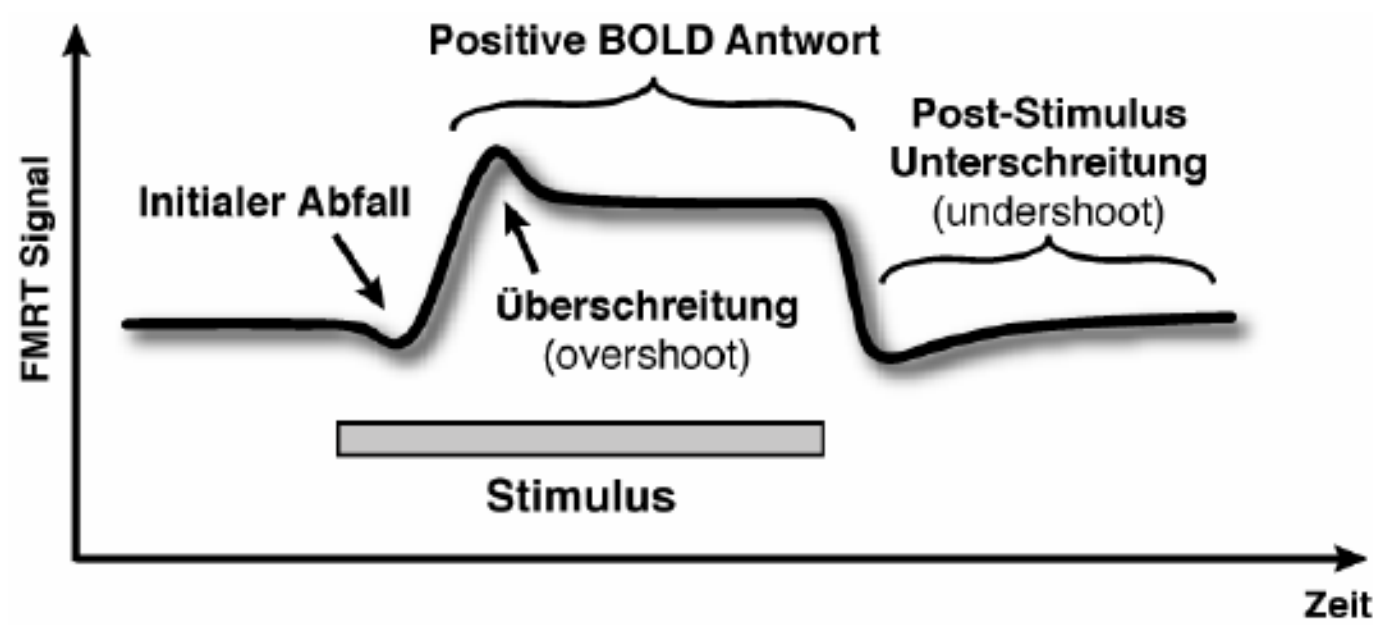

Abbildung 5: Charakteristischer Zeitverlauf des fMRT-Signals bei langer Stimulationsphase (ca. 20 Sekunden). Der initiale Abfall (engl. initial dip) ist bisher nicht zuverlässig messbar, weshalb in fast allen Humanstudien die deutlich stärkere positive BOLD-Antwort analysiert wird. Zu Beginn der positiven Antwort steigt das Signal kurzzeitig auf ein Maximalniveau (Überschreitung, engl. overshoot), und fällt dann auf ein etwas geringeres, konstantes Plateau. Nach Beendigung der Stimulation sinkt das Signal meist unter das ursprüngliche Signalniveau (engl. Baseline), bevor es sich diesem allmählich wieder annähert (Unterschreitungsphase, engl. undershoot) (Goebel und Kriegeskorte 2005, Seite 27). 


\subsection{Ziel der Arbeit}

Nach einem heute weitgehend akzeptierten Modell (vgl. Abbildung 3C) ist das motorische Netzwerk in drei sowohl anatomisch als auch funktionell getrennte Schleifen unterteilt: die sensomotorische, die assoziative und die limbische Schleife. Ihre Anteile übermitteln Informationen zwischen den jeweiligen kortikalen und subkortikalen Arealen. Trotz umfangreicher Forschung gibt es allerdings noch immer Lücken im Verständnis der motorischen Schleifen. Mit meiner Arbeit soll einigen Limitationen vorausgegangener Arbeiten Rechnung getragen werden.

In einer fMRT-Studie am Menschen sollen folgende Punkte Berücksichtigung finden:

- Sowohl der Kortex als auch die Basalganglien sollen erfasst werden

- Die motorischen Aufgaben sollen sowohl die dominante als auch die nichtdominante Hand einbeziehen

- Zur Optimierung von Vergleichbarkeit und Spezifizierung sollen die sensomotorische wie auch die assoziative Schleife mittels einer motorischen Aufgabe dargestellt werden

- es sollen beide Hemisphären beurteilt werden.

Folgende Grundlagen können als bekannt gelten:

- Die assoziative Schleife beinhaltet das Prä-SMA, das anteriore Putamen, den dorsolateralen Präfrontalkortex (DLPFC) sowie wiederum Teile der Insula und des Cerebellums. Diese Hirnregionen sind beteiligt an der Planung und der zeitlichen Koordinierung von Bewegungen. Im Gegensatz zur sensomotorischen Schleife besitzt die assoziative Schleife keine somatotopische Ordnung und Aktivierungen entstehen typischerweise beidseitig im Gehirn. Demnach sollten diese Areale auch bei unilateralen links- oder rechtsseitigen Handbewegungen Aktivität zeigen, es sollten gemeinsame Aktivierungsbereiche resultieren.

- Die sensomotorische Schleife umfasst den SMC, das SMA, das posteriore Putamen, den Thalamus sowie Teile der Insula und des Cerebellums. Diese Hirnregionen sind an streng exekutiven motorischen Aufgaben beteiligt. Es wurde weiter gezeigt, dass sie hauptsächlich unilateral aktiviert werden, nämlich - das Cerebellum ausgenommen - kontralateral zur bewegten Hand. Die sensomotorische Schlei- 
fe ist somatotopisch organisiert. Aktive Hirnregionen sollten für unilaterale Bewegungen der rechten respektive linken Hand keine Überlappungen zeigen.

- Die beiden motorischen Schleifen sollten aufgrund ihrer Lateralitätseigenschaften zu identifizieren sein, d. h. aus einer Trennung der beidseitig von den nur einseitig aktivierten Arealen sollten die oben beschriebenen Schleifen resultieren.

Zur Minimierung der interindividuellen Variabilität und zur Maximierung der Effektstärke wurde ein einfaches Studiendesign entworfen. Für beide Hände wurde ein robustes Fingerbewegungs-Paradigma als Blockdesign aufgesetzt. Um nicht nur rein exekutive, sondern auch assoziative Bewegungscharakteristika einzuschließen, führten die Probanden die Fingerbewegung in einem von ihnen selbst generierten Rhythmus durch.

Mit diesen Kenntnissen ergeben sich zwei Hypothesen:

- Hirnregionen, die bei der unilateralen Bewegung sowohl der linken als auch der rechten Hand beteiligt sind, gehören mutmaßlich zur assoziativen Schleife.

- Hirnregionen, die hauptsächlich während der Bewegung der linken oder aber der rechten Hand Aktivität zeigen, reagieren seitenspezifisch. Vor dem Hintergrund der bekannten somatotopischen Organisation und dem überwiegend unilateralen Aufbau der sensomotorischen Schleife sollten die gefundenen Areale dann letzterer zugeordnet werden können.

Die Identifikation und Differenzierung der verschiedenen motorischen Schleifen können helfen, Bewegungsstörungen und Erkrankungen des motorischen Systems besser zu verstehen und zu untersuchen. Die nicht-invasive und gesundheitlich unbedenkliche fMRTBildgebungstechnik sowie die für den Probanden leicht durchführbare motorische Aufgabe ermöglichen wiederholte Messungen, so dass neben der Beobachtung eines Behandlungserfolges auch die Weiterentwicklung und Evaluation neuer Therapieansätze möglich werden. 


\section{Probanden und Methoden}

\subsection{Probanden}

Untersucht wurden 26 gesunde Jungen (mittleres Alter 11,8 Jahre, +/- 1,1 Jahre, Altersspanne 10,2-14,1 Jahre). Sie waren sämtlich ohne neurologische oder psychiatrische Erkrankungen und ohne aktuelle oder frühere psychoaktive Medikation. Alle 26 Probanden waren Rechtshänder, dies wurde mittels eines Händigkeitstests (Edinburgh Handedness Inventory, (Oldfield 1971)) überprüft und quantifiziert (Mittelwert 94,9, Standardabweichung 14,1; hierbei bedeutet ein Wert von -100 eine komplette Links-, ein Wert von +100 eine komplette Rechtshändigkeit). Im Rahmen einer MRT-Studie zur Untersuchung von Jungen mit TouretteSyndrom im Vergleich zu gesunden Kontrollprobanden waren die Jungen aus örtlichen Schulen rektrutiert worden. Gemäß eines Informed Consent wurde schriftliches Einverständnis von den Jungen und ihren Eltern eingeholt. Die Studie war von der Ethikkommission der Medizinischen Fakultät der Universität Göttingen genehmigt worden und wird den Richtlinien der Deklaration von Helsinki zur klinischen Forschung am Menschen gerecht (Weltärztebund 2001).

Zwei der Probanden mussten nach der Datenerhebung aus der weiteren Studie ausgeschlossen werden, einer aufgrund ausgeprägter motorischer Unruhe während der MRT-Messung, ein zweiter wegen eines durch die MRT-Untersuchung neu entdeckten Tumors in der Orbita.

\subsection{Motorische Aufgabe}

Unser Paradigma bestand aus einer simplen motorischen Aufgabe im Blockdesign. Die Probanden führten mit jeder Hand einzeln ein Tippen des Zeigefingers (sogenanntes Fingertapping) durch. Über eine MR-taugliche LCD-Brille (Resonance Technology, Northridge, Kalifornien, USA) wurde, gleich einer Ampel, ein roter respektive grüner Punkt dargeboten (s. Abbildung 6). Während der rote Punkt angezeigt wurde, hatten die Probanden lediglich die Aufgabe, den Punkt zu fixieren (Ruhebedingung). Wechselte die Anzeige auf den grünen Punkt, so sollten sie mit ihrem Zeigefinger rhythmisch auf eine feste Unterlage tippen (Bewegungsbedingung). Wenngleich die Probanden die Tipp-Bewegung vor Beginn der Untersuchung mit einer Frequenz von ca. 2 Hertz erklärt und gezeigt bekamen, war explizites Ziel die Bewegung nach einem selbst generierten Rhythmus. Alle Probanden übten die 
Aufgabe vor Beginn der MRT-Messungen. Acht Alternationen von Bewegungsbedingung (12 Sekunden) und Ruhebedingung (18 Sekunden) wurden durchgeführt. Mit einer initialen Ruhebedingung von 18 Sekunden betrug die Gesamtmesszeit für ein Experiment 4 Minuten und 18 Sekunden. Alle Probanden konnten die motorische Aufgabe mühelos ausführen. Spiegelbewegungen oder schwerwiegende Fehler konnten von den Untersuchern nicht beobachtet werden.

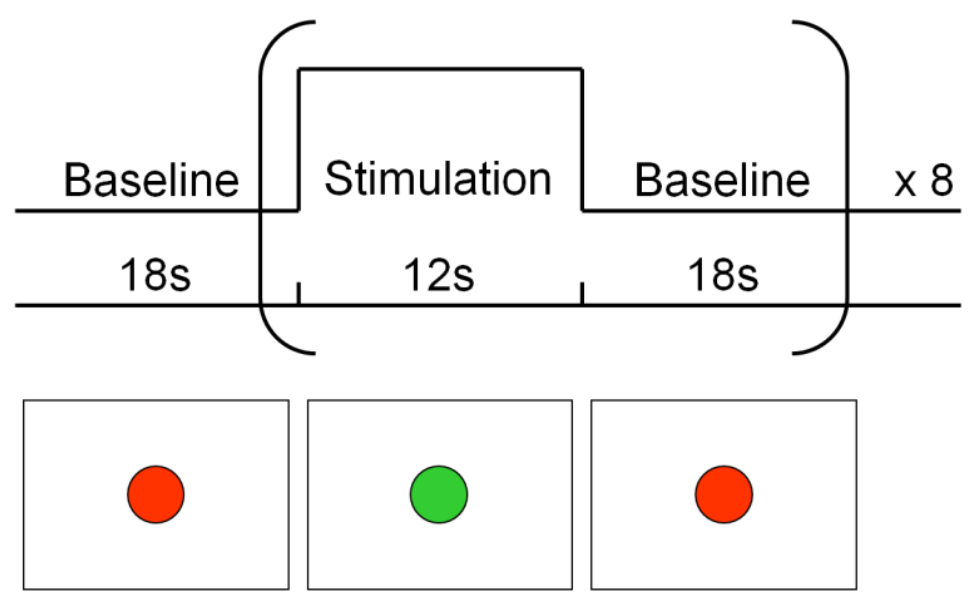

Abbildung 6: Stimulationsprotokoll. Die visuelle Darbietung des roten Punktes gibt die Ruhebedingung vor, der Wechsel zum grünen Punkt markiert den Beginn der Stimulationsphase, die mit dem erneuten Wechsel zum roten Punkt endet. Die Alternation von Stimulationsund Ruhebedingung wurde in unserem Experiment jeweils 8 Mal durchlaufen.

\subsection{Datenerhebung}

Die MRT-Messung erfolgte mit einem 3-Tesla-Kernspintomographen (Magnetom Trio, Siemens Healthcare, Erlangen). Zur Anwendung kam eine Standard-Acht-Kanal-Kopfspule. Die Probanden wurden in Rückenlage untersucht, der Kopf kam in der Mitte des Tomographen zu liegen. Schaumstoffpolster und -keile wurden genutzt, um Bewegungsartefakte zu minimieren. Die Probanden trugen Kopfhörer, einerseits mit dem Ziel des Lärmschutzes, andererseits, um eine Verständigung mit den Untersuchern zwischen den einzelnen Experimenten zu ermöglichen. Herzfrequenz und transkutane Sauerstoffsättigung wurden während des gesamten Versuchs überwacht.

$\mathrm{Zu}$ Beginn wurde ein T1-gewichteter anatomischer Datensatz des gesamten Kopfes mit einer Auflösung von $1 \mathrm{~mm}^{3}$ erhoben (3D Turbo FLASH, Repetitionszeit (TR): 1950 ms, Inversi- 
onszeit: $1100 \mathrm{~ms}$, Echozeit (TE): 3,92 ms, Flipwinkel 12º. Die funktionelle Bildgebung wurde mittels einer T2*-sensitiven Gradienten-Echo-EPI-Technik mit einer Auflösung von 2 x $2 \mathrm{~mm}^{2}$ durchgeführt (TR: $2000 \mathrm{~ms}$, TE: $36 \mathrm{~ms}$, Flipwinkel: 70 , Erfassungsmatrix: $96 \mathrm{x}$ 128). Wir erhielten 129 Datensätze von je 22 Schnittbildern mit $4 \mathrm{~mm}$ Dicke, in einer axialnach-koronaren Kippung. Das gesamte Hirn, insbesondere auch die Basalganglien sowie das Kleinhirn, wurden erfasst.

\subsection{Datenauswertung}

\subsubsection{Auswertung der anatomischen Daten}

Die Datenauswertung wurde mit der Software Brain Voyager QX (Brain Innovation, Maastricht, Niederlande) durchgeführt. Zunächst erfolgte die Analyse der anatomischen Daten. Ziel war es, die individuellen T1-Datensätze so zusammenzuführen, dass ein gemittelter Datensatz im normalisierten Talairach-Raum (Talairach und Tournoux 1988) entsteht. Auf dieses neue, künstlich erzeugte T1-Bild sollten später die funktionellen Daten projiziert werden.

In einem ersten Schritt wurden in den individuellen Datensätzen die Anteriore (AC) und Posteriore (PC) Commissur bestimmt. Um die entstehende Achse erfolgte nun die Rotation der Datensätze in allen drei Ebenen. In einem zweiten Schritt wurde für Größenunterschiede korrigiert. Schließlich wurden die Datensätze gemittelt, so dass ein T1-Bild aus den Daten aller Probanden entstand.

\subsubsection{Auswertung der funktionellen Daten}

\subsubsection{Vorverarbeitung}

Vor der eigentlichen statistischen Analyse funktioneller MRT-Daten ist eine Vorverarbeitung für jede Versuchsperson einzeln und in mehreren Stufen erforderlich.

Zunächst wurde eine 3D-Bewegungskorrektur (3D motion correction) vorgenommen: Kopfbewegungen des Probanden während der Messung führen zur Verschiebung der über die Zeit aufgenommenen Schichten, so dass der Zeitverlauf eines Voxels (d.h. der kleinsten dreidimensionalen Einheit, der eine Signalintensität zugeordnet wird) möglicherweise fehlerhafte Signale enthält. Um diese Fehlerquelle zu vermeiden, werden die Schichten durch Translation entlang der drei Achsen und Rotation in den drei Ebenen einer Ausgangsschicht angepasst. 
Um die Erfassungsverzögerung auszugleichen, d.h. zeitliche Differenzen, die durch die schichtweise Aufnahme des Gehirns entstehen, wurde eine Messzeit-Korrektur (slice scan time correction) durchgeführt.

Die räumliche Glättung (spatial smoothing) ist für die Analyse einer Probandengruppe sinnvoll. Ziel ihrer Anwendung ist die Anpassung korrespondierender Hirnareale in den Datensätzen der einzelnen Probanden mit ihren minimalen individuellen Unterschieden, außerdem die Erhöhung der statistischen Validität durch Betonung der signalintensiven und Dämpfung der signalschwachen Bereiche. Die Signalintensität eines Voxels wird hierbei mittels eines „Gaußschen Filters“ auf die umgebenden Voxel „verwischt“. Der Gaußsche Filter ist eine Normalverteilung definierter Breite (hier: $5 \mathrm{~mm}$ Halbwertsbreite oder full width at half maximum, FWHM).

Letzter Schritt der Datenvorverarbeitung war die Basislinienkorrektur (linear trend removal). Schaut man sich den Zeitverlauf für ein Voxel an, so ist neben den relativen, aufgabenbedingten Signalveränderungen meist ein „Drift“ des absoluten Signals zu erkennen. Da dieser Drift die Auswertung verfälschen könnte, nutzt man einen entsprechenden Filter zur Korrektur.

Anschließend wurden die einzelnen funktionellen Datensätze mittels einer Überführung in den Talairach-Raum einerseits und in eine Voxelgröße von 3 × 3 × $3 \mathrm{~mm}^{3}$ andererseits standardisiert und auf den anatomischen Datensatz koregistriert.

\subsubsection{Das Allgemeine Lineare Modell}

Nach Abschluss der Datenvorbereitung konnte nun die eigentliche Analyse der fMRT-Daten erfolgen. Diese erfolgt üblicherweise voxelweise; das bedeutet, dass die Erstellung des Signal-Zeit-Verlaufes für jedes Voxel eines jeden Probanden einzeln erfolgt. Hierfür geht man von der Annahme aus, dass ein linearer Zusammenhang besteht zwischen dem tatsächlich gemessenen Signal $y$ eines Voxels $v$ zum Zeitpunkt $t$ und einem zuvor erstellten theoretischen Modell. Dieses Modell wiederum ergibt sich aus dem Stimulationsprotokoll einerseits und dem Wissen über die physiologischen Prozesse, ausgedrückt in der „Hämodynamischen Antwort-Funktion", andererseits.

Der postulierte lineare Zusammenhang wird ausgedrückt im „Allgemeinen linearen Modell“ (general linear model, GLM):

$$
y_{t}=\beta_{1} x_{1}+\beta_{2} x_{2}+\ldots+\beta_{n} x_{n}+a
$$


Demnach ist das gemessene Signal $y$ eines Voxels die Summe aus spezifisch gewichteten Einzelfaktoren $x$ und einer Konstante $a$ (s. Abbildung 7). Die Einzelfaktoren stellen die unabhängigen Variablen (in unserem Falle die durch die Fingerbewegungen veränderte Hirnaktivität) des Versuchsaufbaus dar. Für die Berechnung der spezifischen Gewichtungen ( $\beta$-Werte) wird nun der gemessene Signal-Zeit-Verlauf eines Voxels verglichen mit dem theoretischen Modell. Über eine Modulation der $\beta$-Werte wird angestrebt, den tatsächlichen Signal-ZeitVerlauf mit dem theoretischen Modell in Übereinstimmung zu bringen. Je höher die erreichbare Übereinstimmung, desto besser ist der gemessene Signal-Zeit-Verlauf (also das BOLDSignal) durch das theoretische Modell (also die Stimulation) zu erklären. Ein $\beta$-Wert ist demnach ein voxelspezifischer, zeitunabhängiger Schätzer für die Güte des theoretischen Modells einerseits und für den Einfluss der einzelnen Variablen andererseits.

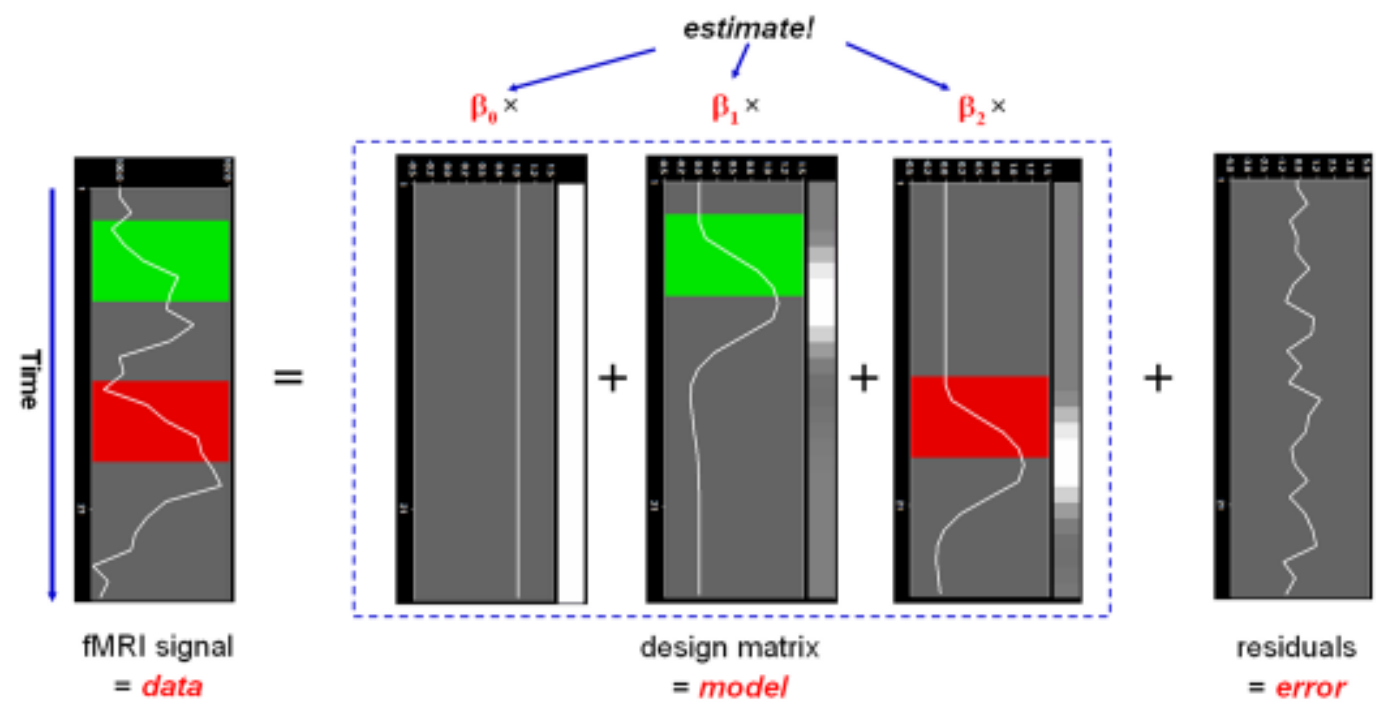

Abbildung 7: Das Allgemeine Lineare Modell in einem vereinfachten Schema. Das gemessene Signal eines Voxels über die Zeit (linke Spalte) wird in seine Komponenten aufgegliedert. Ziel ist, durch Modulation der ß-Schätzwerte die Einzelfaktoren (Mitte) so zu gewichten, dass - unter Berücksichtung von Residuen (rechte Spalte) - der gemessene Signalverlauf optimal mit dem theoretischen Modell erklärt werden kann.

(www.brainvoyager.com/bvqx/doc/UsersGuide/WebHelp/BrainVoyagerQXUsersGuide.htm)

Die Berechnung einer t-Statistik erfolgt nun für jedes Voxel eines jeden Probanden. Die Ergebnisse werden auf den zugehörigen anatomischen Datensatz projiziert, hierbei werden nur diejenigen Voxel farblich markiert, die eine gegebene statistische Schwelle überschreiten. 


\subsubsection{Auswertung der Gruppendaten}

Nach der individuellen Datenanalyse erfolgt die Gruppenanalyse. Die funktionellen Datensätze der Probanden werden gemeinsam erfasst, und die Ergebnisse der Berechnungen anschließend auf den gemittelten, Talairach-normalisierten anatomischen Gruppen-Datensatz projiziert.

Für die gemeinsame Erfassung der Gruppendaten gibt es in der fMRI-Datenanalyse zwei verschiedene Herangehensweisen. In der Berechnung der „Fixen Effekte“ (fixed effects analysis) werden die Daten der Probanden statistisch so betrachtet, als seien sie zwischen den einzelnen Versuchspersonen stabil, d.h. Unterschiede zwischen den Datensätzen werden nicht durch individuelle Variabilität, sondern durch zufälliges Rauschen erklärt. Die statistische Aussagekraft ist bei dieser Methode vergleichsweise gering, die Ergebnisse können nicht auf die Gesamtpopulation verallgemeinert werden. Bei der Berechnung der „Zufälligen Effekte“ (random effects analysis) hingegen wird berücksichtigt, dass die gleiche Stimulation unterschiedliche Reaktionen bei den verschiedenen Probanden auslösen könnte. Der Variabilität zwischen den Probanden wird demnach Rechnung getragen. Entsprechend sind die Ergebnisse dieser Herangehensweise auf die Gesamtpopulation, der die Probanden entnommen wurden, verallgemeinerbar.

Für unsere Arbeit wurde eine Analyse nach dem Random-Effects-Modell berechnet. Das Allgemeine Lineare Modell ermöglicht es nun, für verschiedene Fragestellungen Kontraste zu erstellen. Zunächst wurden die Haupteffekte für die Bewegungsbedingung jeder Hand ermittelt (linke Hand versus Ruhebedingung, rechte Hand versus Ruhebedingung). In einem nächsten Schritt wurde eine Konjunktions-Analyse berechnet. Hier werden all die Voxel farbig markiert, die sowohl bei der Bewegung der linken als auch der rechten Hand aktiviert werden. Schließlich erstellten wir eine Kontrast-Analyse. Diese zeigt Voxel an, die bei der Bewegung der linken Hand stärker aktiviert werden als bei der Bewegung der rechten Hand, bzw. umgekehrt. Während bei der Analyse der Haupteffekte und der Konjunktion definitionsgemäß nur positive Werte Beachtung finden (vgl. Abbildung 9), gibt es bei der Kontrast-Analyse positive und negative Ergebnisse: die Dominanz der einen Hand wird positiv, die der anderen Hand negativ kodiert (vgl. Abbildung 12).

Den Schwellenwert für die resultierenden statistischen Karten legten wir für alle Gruppenanalysen gleichermaßen bei $\mathrm{p}<0,001$ (entsprechend $t>3,77$ ) fest. 


\subsubsection{Korrektur für multiple Vergleiche}

Zur Korrektur für multiple Vergleiche nutzen wir den „Cluster size thresholding“-Ansatz von Forman (Forman et al. 1995). Dies einerseits, da dieser Ansatz der Fragestellung nach relevant großen Aktivierungszonen am besten gerecht wird, andererseits, weil so die p-Werte für verschiedene Analysen festgelegt und konstant gehalten werden können, wodurch ein Vergleich untereinander möglich wird. Bei dem gewählten $\mathrm{p}$-Wert von $\mathrm{p}<0,001$ ergab sich die Mindestgröße für ein als relevant zu bewertendes Cluster von 4 funktionellen Voxeln (entsprechend $108 \mathrm{~mm}^{3}$ ).

Wie oben beschrieben, wurden die individuellen Talairach-standardisierten anatomischen T1Datensätze gemittelt. Auf diesen neuen Gruppen-T1-Datensatz wurden die funktionellen Aktivierungskarten projiziert, nachdem sie mittels Interpolation in eine 1 x 1 x $1 \mathrm{~mm}^{3}$ Auflösung überführt wurden. Aktivierungscluster wurden definiert als zusammenhängende aktivierte Voxel; ihre räumliche Ausdehnung sowie ihre lokalen Maxima wurden ermittelt. Die Zuordnung zu anatomischen Hirnregionen wurde mithilfe des Talairach Daemon vorgenommen (http://www.talairach.org/client.html), die Koordinaten des lokalen Maximums waren hierfür ausschlaggebend. 


\section{Ergebnisse}

\subsection{Haupteffekte}

Unsere Auswertung ergab die abgebildeten Aktivierungskarten (s. Abbildungen 8 - 14). Die Karten der Haupteffekte für linksseitige bzw. rechtsseitige Zeigefingerbewegung zeigten sich weitgehend spiegelbildlich. Es ergaben sich keine wesentlichen Unterschiede der Muster für die Bewegung der dominanten im Vergleich zur nicht-dominanten Hand (s. Abbildung 8 und Tabelle 1).

Das Netzwerk umfasste kortikale Hirnregionen wie den kontralateral zur bewegten Hand gelegenen primären SMC, SMA, Prä-SMA und den anterioren zingulären Kortex (ACC) jeweils beidseitig, außerdem bilaterale Anteile der Basalganglien, den kontralateralen Thalamus und das ipsilaterale Cerebellum, sowie schließlich beidseitig die Inselrinde und den kontralateralen sekundär somatosensorischen Kortex (SII). Die Zeigefingerbewegung der dominanten rechten Hand rief außerdem beidseitige Aktivierung im DLPFC vor, während dies für die linke Hand ausschließlich im kontralateralen DLPFC der Fall war. In den Basalganglien zeigte sich ein klares Muster mit Aktivierung des kontralateral zur bewegten Seite gelegenen Putamen über die gesamte rostrokaudale Achse, außerdem von anterioren Anteilen des ipsilateralen Putamens sowie des kontralateralen GP. Bei der Bewegung des linken Zeigefingers konnte auch Aktivierung im ipsilateralen GP gefunden werden.

Verminderte Aktivierung im Vergleich zur Ruhebedingung wurde in postzentral gelegenen, ipsilateralen Regionen gesehen.

Legende zu Tabelle 1, S. 27: Haupteffekte; Gruppenanalyse nach dem Random-effectsModell. Signifikanzniveau $p<0,001$, Korrektur für multiple Vergleiche nach dem Cluster-sizeThresholding-Ansatz bei $108 \mathrm{~mm}^{3}$. Die Koordinaten nach Talairach sind in $\mathrm{mm}$ angegeben. $\mathrm{L}$ = links; $R$ = rechts. a) summierte Aktivierung zusammenhängender Areale; die beteiligten Regionen sind in den jeweils folgenden Zeilen aufgeführt. 
Tabelle 1 (Legende auf Seite 26)

\begin{tabular}{|c|c|c|c|c|c|c|c|c|c|c|c|}
\hline \multirow{3}{*}{ Region } & \multicolumn{6}{|c|}{ Haupteffekte. Aktivierte Hirnregionen bei unilateraler Fingerbewegung. } & \multicolumn{5}{|c|}{ Fingerbewegung der rechten Hand } \\
\hline & \multirow{2}{*}{ Hemisphäre } & \multicolumn{3}{|c|}{ Talairach } & \multirow{2}{*}{$\begin{array}{c}\text { Max } \\
\mathrm{t}-\text { Wert }\end{array}$} & \multirow{2}{*}{$\begin{array}{l}\text { Größe } \\
\left(\mathrm{mm}^{3}\right)\end{array}$} & \multicolumn{3}{|c|}{ Talairach } & \multirow{2}{*}{$\begin{array}{c}\text { Max } \\
\text { t-Wert }\end{array}$} & \multirow{2}{*}{$\begin{array}{l}\text { Größe } \\
\left(\mathrm{mm}^{3}\right)\end{array}$} \\
\hline & & $x$ & $y$ & $z$ & & & $x$ & $\mathrm{y}$ & $\mathrm{z}$ & & \\
\hline \multicolumn{12}{|l|}{ Kortikale motorische Areale } \\
\hline \multirow[t]{2}{*}{ Primär sensomotorischer Kortex } & $\mathrm{L}$ & - & - & - & - & - & -30 & -22 & 52 & 13.81 & 11342 \\
\hline & $\mathrm{R}$ & 30 & -25 & 58 & 14.45 & 9769 & - & - & - & - & - \\
\hline $\begin{array}{l}\text { Supplementär-motorischer Kortex } \\
\text { prä-Supplementär-motorischer }\end{array}$ & $L / R$ & -6 & -10 & 55 & 7.06 & 7936 a) & -6 & -13 & 55 & 6.47 & 6947 a) \\
\hline Kortex & $L / R$ & 0 & 2 & 46 & 6.4 & & 3 & 5 & 49 & 6.53 & \\
\hline Anteriorer Gyrus cinguli & $L / R$ & 3 & 17 & 34 & 4.76 & & -3 & -4 & 40 & 6.87 & \\
\hline \multirow[t]{2}{*}{ Dorsolateraler Präfrontalkortex } & $\mathrm{L}$ & - & - & - & - & - & -30 & 35 & 31 & 5.03 & 508 \\
\hline & $\mathrm{R}$ & 30 & 41 & 37 & 5.22 & 586 & 30 & 41 & 34 & 6.95 & 2203 \\
\hline \multirow{2}{*}{ Primär somatosensorischer Kortex } & $\mathrm{L}$ & -24 & -37 & 61 & -6.04 & 4270 & - & - & - & - & - \\
\hline & $\mathrm{R}$ & - & - & - & - & - & 30 & -37 & 46 & -5.85 & 2729 \\
\hline \multirow[t]{2}{*}{ Posteriorer Parietalkortex } & $\mathrm{L}$ & - & - & - & - & - & -39 & -22 & 22 & 5.98 & 1792 \\
\hline & $\mathrm{R}$ & 39 & -22 & 25 & 4.86 & 164 & - & - & - & - & - \\
\hline \multicolumn{12}{|l|}{ Insula und subkortikale Areale } \\
\hline Insula & $\mathrm{L}$ & -39 & 2 & 4 & 5.69 & 6027 a) & -45 & 2 & 7 & 7.77 & 2163 \\
\hline Rostrales Putamen & $L$ & -18 & 8 & 4 & 4.76 & & -18 & 5 & 16 & 6.05 & 8866 a) \\
\hline Kaudales Putamen & $\mathrm{L}$ & - & - & - & - & - & -24 & -7 & 7 & 9.31 & \\
\hline Globus pallidus & L & -18 & -7 & 7 & 5.79 & & -18 & -7 & 7 & 5.63 & \\
\hline Thalamus & L & - & - & - & - & - & -15 & -19 & 10 & 8.72 & \\
\hline Nucleus subthalamicus & $L$ & - & - & - & - & - & -3 & -19 & -8 & 5.13 & \\
\hline Insula & $\mathrm{R}$ & 42 & 5 & 10 & 6.72 & 17751 a) & 36 & 14 & 4 & 8.01 & 4432 \\
\hline Rostrales Putamen & $\mathrm{R}$ & 18 & 8 & 10 & 6.25 & & 15 & 5 & 7 & 6.06 & 985 \\
\hline Kaudales Putamen & $\mathrm{R}$ & 24 & -10 & 4 & 6.44 & & - & - & - & - & - \\
\hline Globus pallidus & $\mathrm{R}$ & 18 & -7 & 1 & 9.05 & & - & - & - & - & - \\
\hline Thalamus & $\mathrm{R}$ & 15 & -19 & 7 & 9.81 & & - & - & - & - & - \\
\hline Nucleus subthalamicus & $\mathrm{R}$ & 9 & -19 & -5 & 4.91 & & - & - & - & - & - \\
\hline \multirow[t]{2}{*}{ Cerebellum } & $\mathrm{L}$ & -12 & -49 & -14 & 8.73 & 7818 & - & - & - & - & - \\
\hline & $\mathrm{R}$ & - & - & - & - & - & 12 & -46 & -14 & 8.9 & 7051 \\
\hline
\end{tabular}




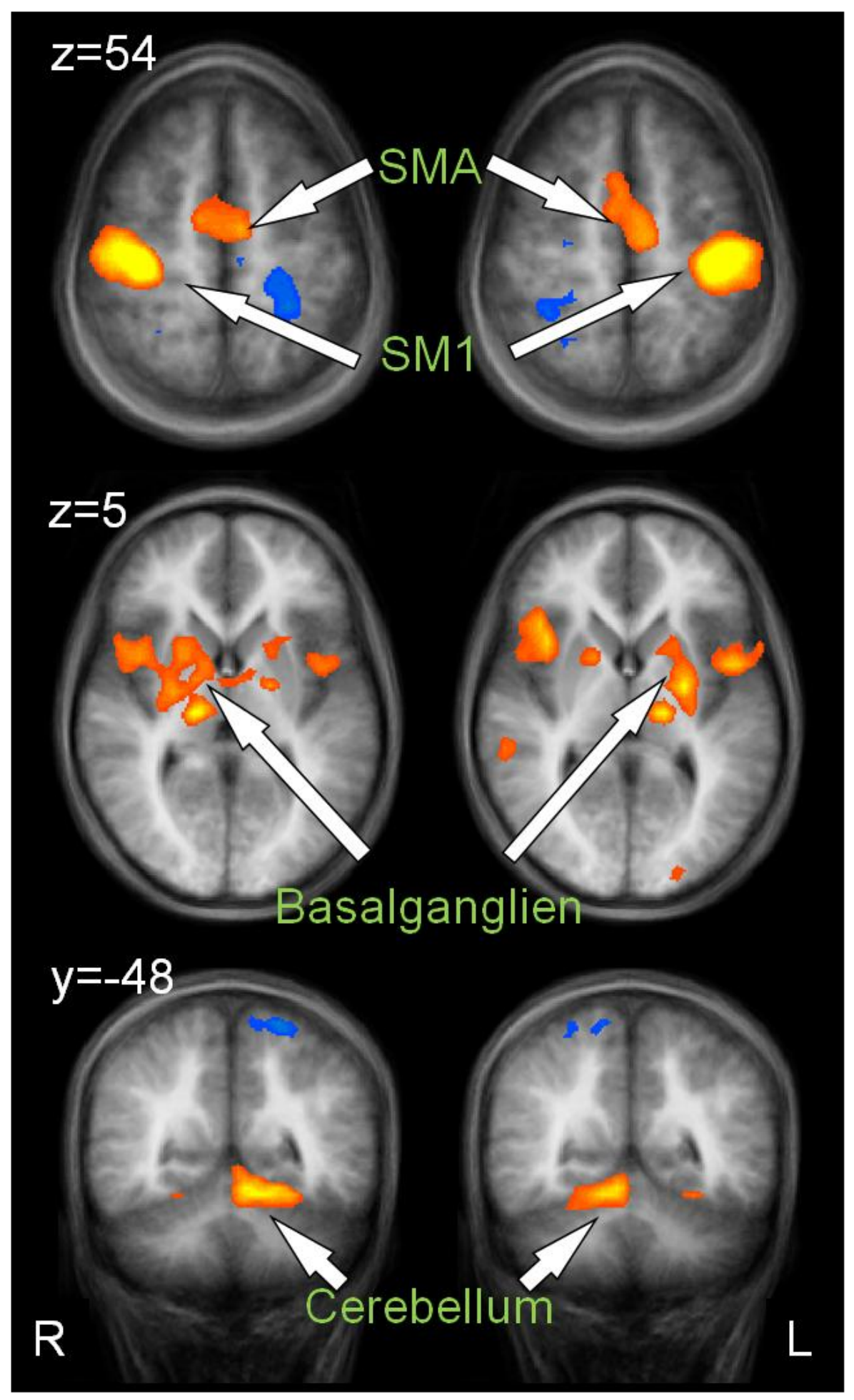

Abbildung 8: Haupteffekte. Gruppenanalyse nach dem Random-Effects-Modell ( $n=22)$ während linkshändiger (linke Spalte) respektive rechtshändiger (rechte Spalte) Bewegung des Zeigefingers im Vergleich zur Ruhebedingung. Die Aktivierungskarten wurden auf ein gemitteltes und Talairach-standardisiertes anatomisches T1-Gruppenbild projiziert.

Signifikante Signalveränderungen ( $p<0,001$; korrigiert für multiple Vergleiche) sind in Gelb/Rot (positive Signalveränderung) bzw. Grün/Blau (negative Signalveränderung) dargestellt. $L=$ links; $R=$ rechts. Die Schnittebene im Talairach-Koordinatensystem ist in $\mathrm{mm}$ angegeben. 


\subsection{Konjunktionsanalyse}

Um Hirnregionen zu identifizieren, die sowohl während der Bewegung der linken wie auch der rechten Hand Aktivierung zeigten, rechneten wir eine Konjunktions-Analyse (siehe Tabelle 2). Wir erhielten ein weitgehend symmetrisches Muster (siehe Abbildung 9).

In den Bereichen der SMA und der Prä-SMA fand sich eine Signalsteigerung, die sich über die rostralen Anteile des Gyrus frontalis medialis beidseits zusammenhängend erstreckte. In den Basalganglien kam eine ebenfalls bilaterale Aktivierung des rostralen Putamens zur Darstellung. Auch mediale Anteile des Cerebellums zeigten Aktivierung. Weitere Signalsteigerungen waren in der Insula beidseits sowie im rechten DLPFC zu finden. Der Globus pallidus war lediglich in der linken Hemisphäre aktiviert.

Tabelle 2: Konjunktionsanalyse; Gruppenanalyse nach dem Random-Effects-Modell, Signifikanzniveau $\mathrm{p}<0,001$, Korrektur für multiple Vergleiche nach dem Cluster-sizeThresholding-Ansatz bei $108 \mathrm{~mm}^{3}$. Die Koordinaten nach Talairach sind in $\mathrm{mm}$ angegeben. $\mathrm{L}$ $=$ links; $\mathrm{R}=$ rechts; SMAr = rostrales supplementär-motorisches Areal; DLPFC = dorsolateraler Präfrontalkortex. a) summierte Aktivierung zusammenhängender Areale; die beteiligten Regionen sind in den jeweils folgenden Zeilen aufgeführt. b) Die Größe dieser Aktivierung ist unterhalb der Cluster-size-Schwelle.

\begin{tabular}{|c|c|c|c|c|c|c|}
\hline \multirow{2}{*}{ Region } & \multirow{2}{*}{ Hemisphäre } & \multicolumn{3}{|c|}{ Talairach } & \multirow{2}{*}{$\begin{array}{c}\text { Max } \\
\text { t-Wert }\end{array}$} & \multirow{2}{*}{$\begin{array}{l}\text { Größe } \\
\left(\mathrm{mm}^{3}\right)\end{array}$} \\
\hline & & $x$ & $y$ & $z$ & & \\
\hline SMAr & $L / R$ & -6 & -13 & 55 & 6.47 & 4278 a) \\
\hline Prä-SMA & $L / R$ & 0 & 2 & 46 & 6.35 & \\
\hline \multirow[t]{2}{*}{ Rostrales Putamen } & L & -18 & 8 & 13 & 4.77 & 1618 \\
\hline & $\mathrm{R}$ & 18 & 5 & 7 & 5.32 & 786 \\
\hline Globus pallidus & L & -18 & -7 & 7 & 5.63 & 185 \\
\hline Cerebellum & $\mathrm{L} / \mathrm{R}$ & 0 & -55 & -14 & 5.03 & 402 \\
\hline \multirow[t]{2}{*}{ Insula } & L & -42 & 2 & 4 & 5.13 & 683 \\
\hline & $\mathrm{R}$ & 39 & 8 & 4 & 5.36 & 1827 \\
\hline \multirow[t]{2}{*}{ DLPFC } & L & -30 & 41 & 25 & 4.08 & $64 \mathrm{~b})$ \\
\hline & $\mathrm{R}$ & 30 & 41 & 37 & 5.22 & 464 \\
\hline
\end{tabular}




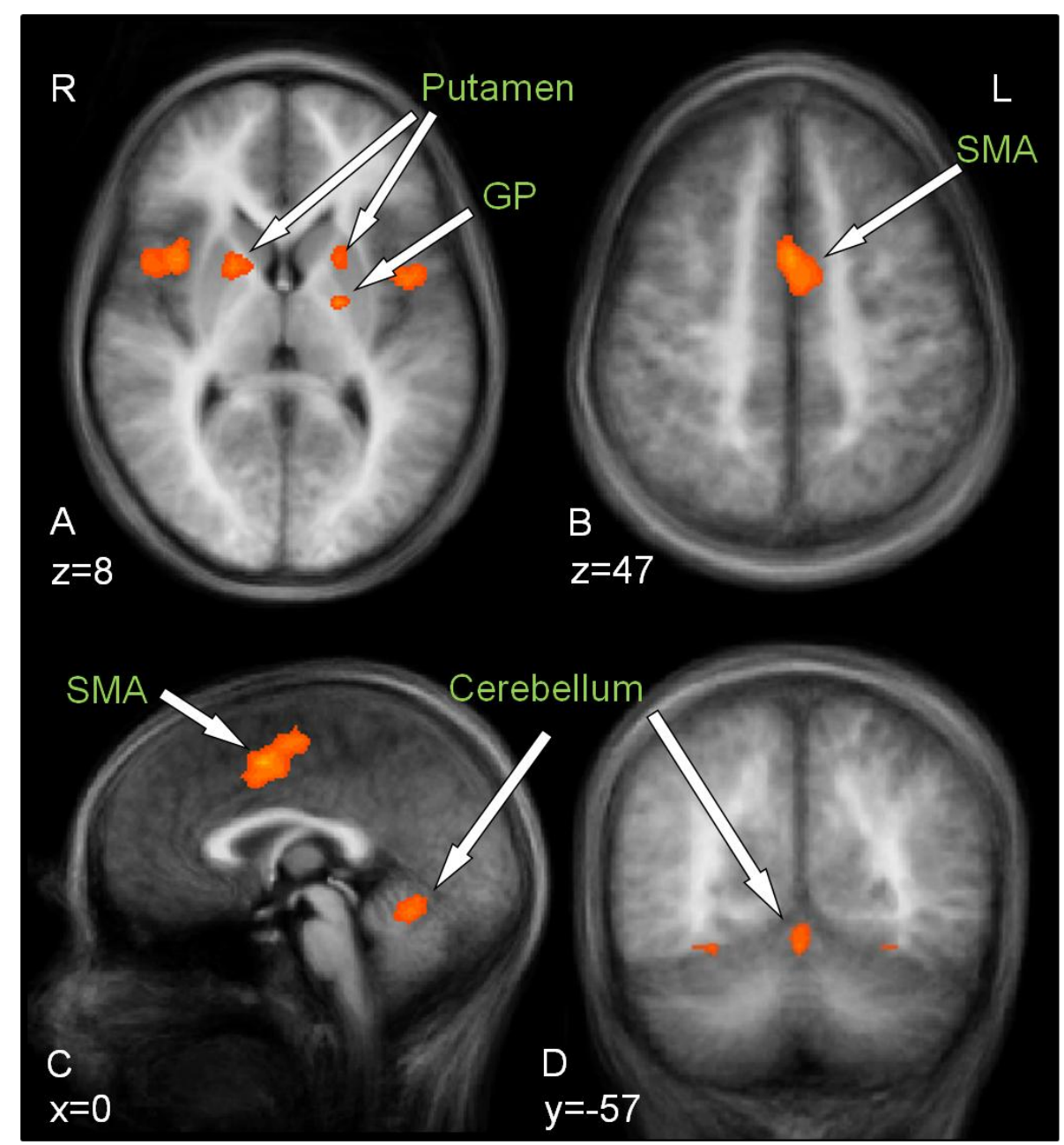

Abbildung 9: Konjunktionsanalyse; Gruppenanalyse nach dem Random-Effects-Modell. Die funktionellen Karten wurden auf ein gemitteltes und Talairach-standardisiertes anatomisches T1-Gruppenbild projiziert. A: Aktivierung in den rostralen Putamina beidseits, im linken Globus pallidus sowie der Insula beidseits. B: Konfluierende Aktivierung in der Prä-SMA beider Hemisphären. C und D: Mediale Aktivierung im Kleinhirn, in C auch der Prä-SMA. A und B sind Transversalschnitte, C ist ein Sagittalschnitt, D ein Frontalschnitt. Die Schnittebene im Talairach-Koordinatensystem ist in $\mathrm{mm}$ angegeben.

Signifikante Signalveränderungen ( $p<0,001$; korrigiert für multiple Vergleiche) sind in Gelb/Rot dargestellt. $L$ = links; $R$ = rechts.

Zur genaueren Darstellung ermittelten wir Zeitverläufe, die das BOLD-Signal für die einzelnen Regionen anzeigen. Es zeigten sich für die rostrale SMA sowie für das rostrale Putamen nahezu identische Zeitverläufe für die Bewegung der ipsi- und der kontralateralen Hand (s. Abbildungen 10 und 11). 


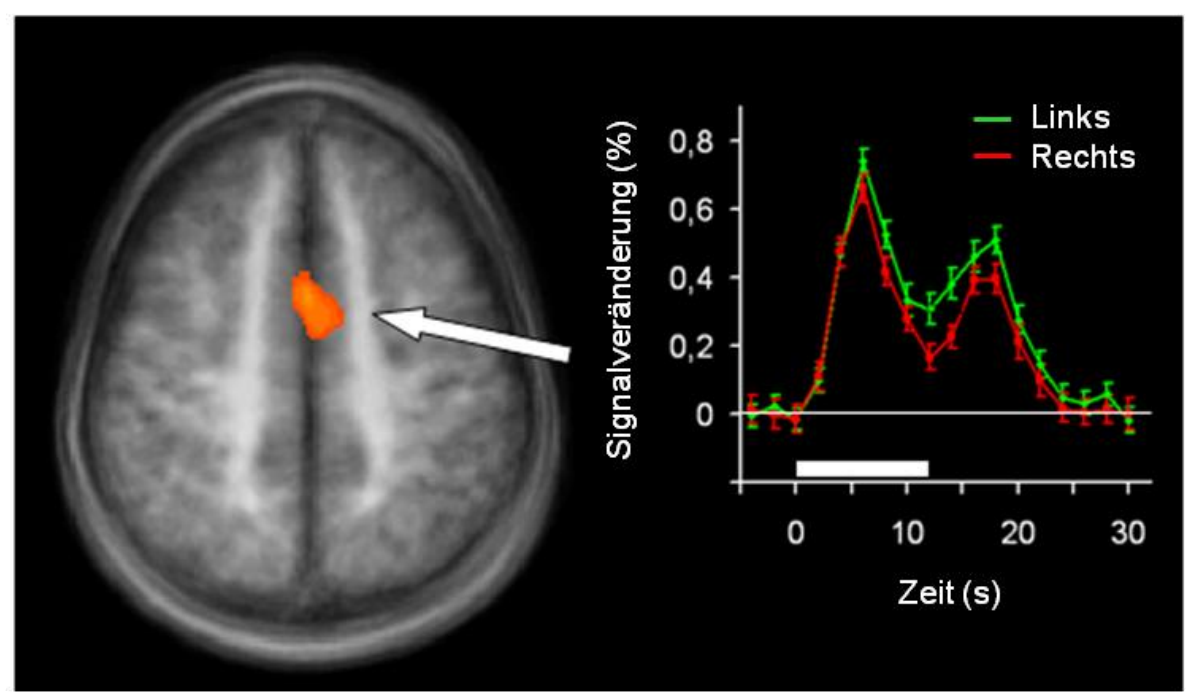

Abbildung 10: Die rostrale SMA in der Konjunktionsanalyse mit Signal-Zeit-Verlauf. Hierfür wird das farblich kodierte und durch den Pfeil markierte Areal funktionell wie ein einziges Voxel betrachtet, dessen Signalveränderung (in \%) in Funktion der Zeit (in s) aufgetragen wird (Signifikanzniveau $\mathrm{p}<0,001$; korrigiert für multiple Vergleiche). Der weiße Balken markiert die Phase der Fingerbewegung. Die grüne Kurve stellt den Verlauf für linkshändige, die rote Kurve für rechtshändige Fingerbewegung dar.

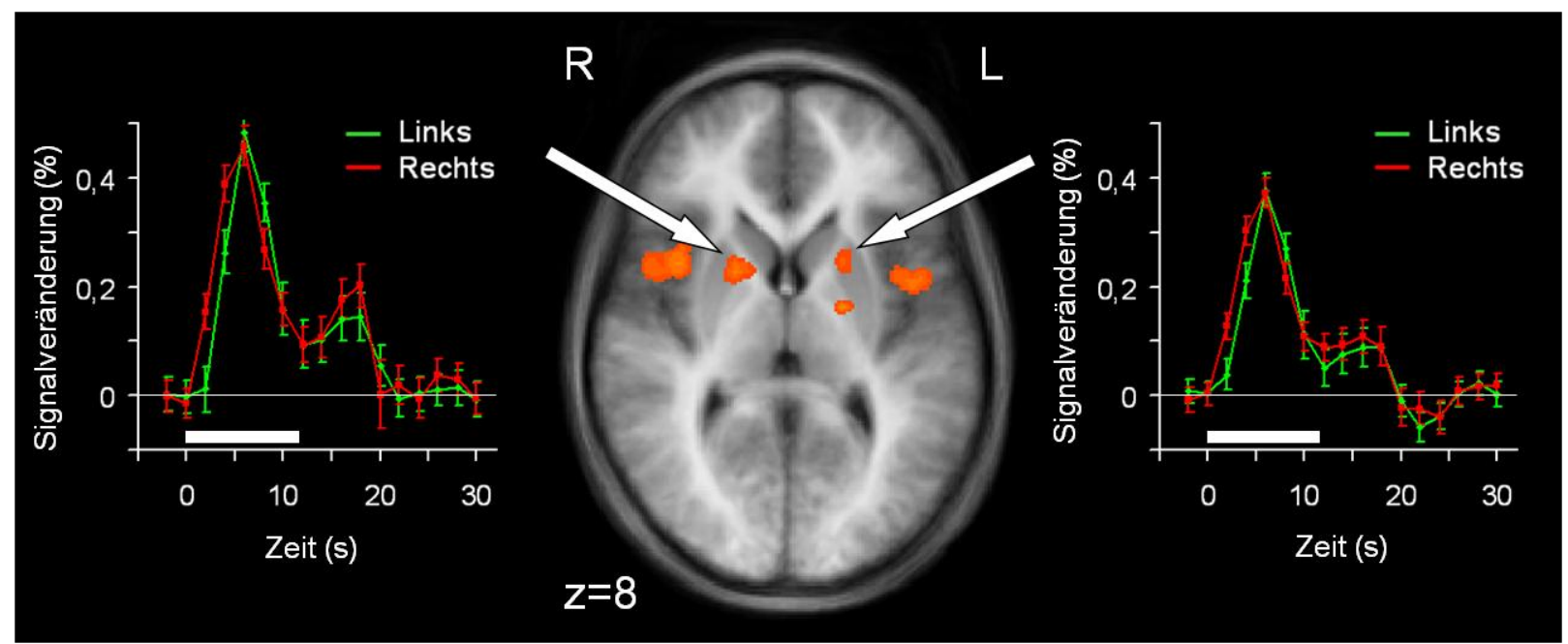

Abbildung 11: Die Putamina in der Konjunktionsanalyse mit Signal-Zeit-Verläufen. Analysiert wurden die farblich kodierten Areale im rostralen Putamen beidseits (durch Pfeile markiert). Übrige Angaben wie in Abbildung 10. 


\subsection{Kontrastanalyse}

Im nächsten Schritt erfolgte eine Kontrast-Analyse (s. Tabelle 3). Diese diente zur Abbildung von Hirnregionen, die seitenspezifisch auf die Bewegung des Zeigefingers der linken respektive der rechten Hand reagierten. Wir erhielten ein weitgehend spiegelbildliches Muster (s. Abbildung 12).

Während die Aktivierung im Cerebellum ipsilateral zur Bewegung war, kamen alle anderen beobachteten Signalsteigerungen in der zur Bewegung kontralateralen Hemisphäre zur Darstellung. Hier identifizierten wir den SMC, kaudale Anteile des SMA und des Putamens, den Thalamus sowie SII, außerdem Anteile der ipsilateralen cerebellären Hemisphäre.

Tabelle 3: Kontrastanalyse; Gruppenanalyse nach dem Random-Effects-Modell. Signifikanzniveau $\mathrm{p}<0,001$, Korrektur für multiple Vergleiche nach dem Cluster-sizeThresholding-Ansatz bei $108 \mathrm{~mm}^{3}$. Die Koordinaten nach Talairach sind in $\mathrm{mm}$ angegeben. $\mathrm{L}$ = links; $R$ = rechts; SMAc = kaudales supplementär-motorisches Areal. a) summierte Aktivierung zusammenhängender Areale; die beteiligten Regionen sind in den jeweils folgenden Zeilen aufgeführt. b) Die Größe dieser Aktivierung ist unterhalb der Cluster-size-Schwelle.

\begin{tabular}{|c|c|c|c|c|c|c|}
\hline \multicolumn{7}{|c|}{ Kontrastanalyse. Differentielle Effekte von linkshändiger versus rechtshändiger Fingerbewegung. } \\
\hline \multirow{2}{*}{$\begin{array}{c}\text { Kontrast } \\
\text { Region }\end{array}$} & \multirow{2}{*}{ Hemisphäre } & \multicolumn{3}{|c|}{ Talairach } & \multirow{2}{*}{$\begin{array}{c}\text { Max } \\
\text { t-Wert }\end{array}$} & \multirow{2}{*}{$\begin{array}{l}\text { Größe } \\
\left(\mathrm{mm}^{3)}\right.\end{array}$} \\
\hline & & $x$ & y & z & & \\
\hline \multicolumn{7}{|c|}{ Fingerbewegung rechtshändig > linkshändig } \\
\hline Primär sensomotorischer Kortex & L & -30 & -25 & 49 & -12.55 & 13186 \\
\hline SMAc & L & -6 & -22 & 49 & -5.63 & 952 \\
\hline Thalamus & L & -15 & -19 & 4 & -10.16 & 912 \\
\hline Kaudales Putamen & L & -30 & -10 & 4 & -8.99 & 1545 a) \\
\hline Posteriorer Parietalkortex & L & -36 & -19 & 22 & -5.47 & \\
\hline Cerebellum & $R$ & 12 & -46 & -14 & -7.63 & 2047 \\
\hline \multicolumn{7}{|c|}{ Fingerbewegung linkshändig > rechtshändig } \\
\hline Primär sensomotorischer Kortex & $\mathrm{R}$ & 30 & -25 & 49 & 10.21 & 10819 \\
\hline SMAC & $\mathrm{R}$ & 6 & -25 & 46 & 4.15 & $41 \mathrm{~b})$ \\
\hline Thalamus & $\mathrm{R}$ & 15 & -19 & 10 & 9.1 & 5619 a) \\
\hline Kaudales Putamen & $\mathrm{R}$ & 27 & -10 & 4 & 7.13 & \\
\hline Posteriorer Parietalkortex & $\mathrm{R}$ & 39 & -22 & 22 & 7.63 & \\
\hline Cerebellum & $\mathrm{L}$ & -12 & -49 & -14 & 7.27 & 2347 \\
\hline
\end{tabular}




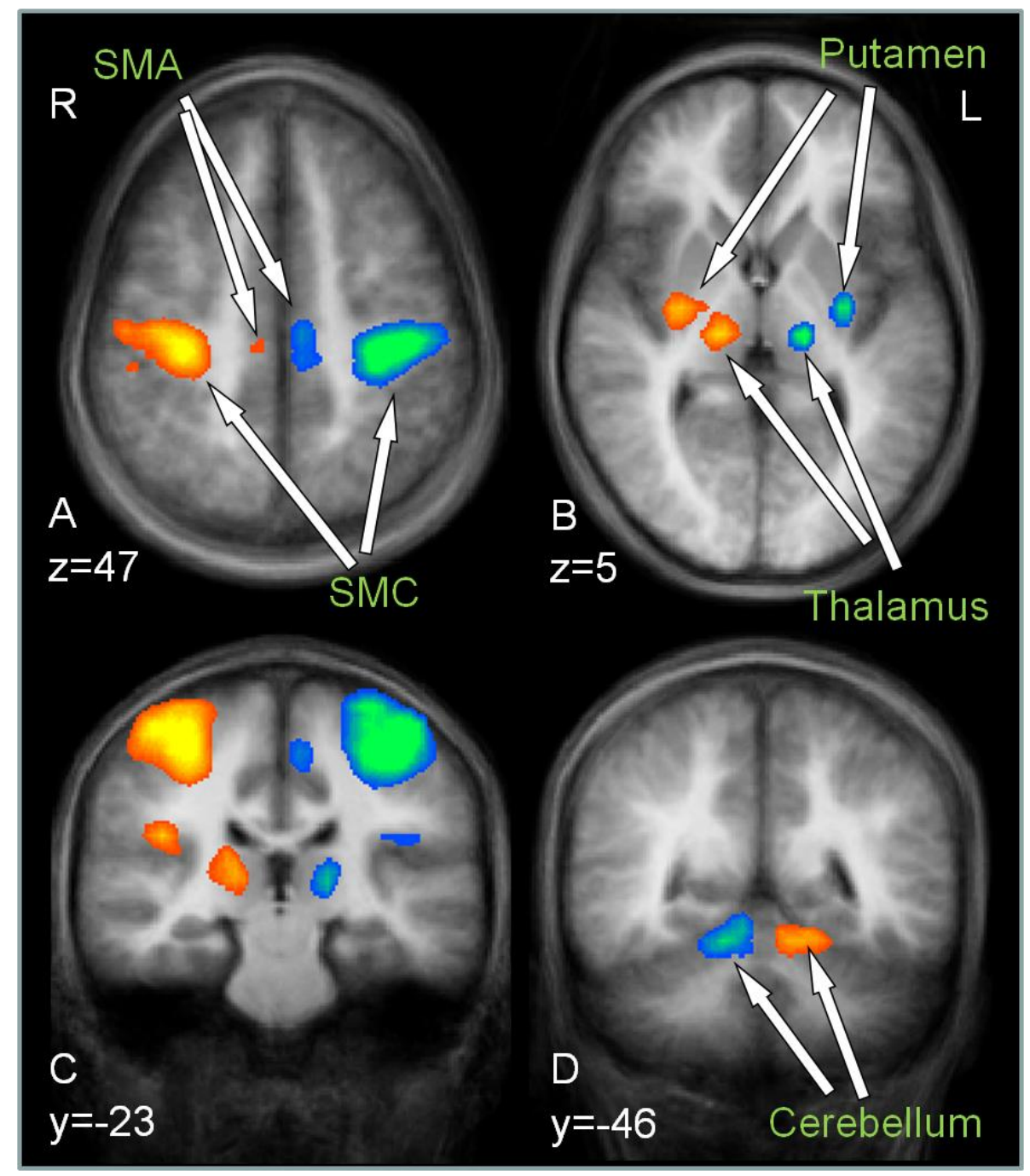

Abbildung 12: Kontrastanalyse; Gruppenanalyse nach dem Random-Effects-Modell. Hirnregionen, die während der linkshändigen Fingerbewegung stärker aktivieren, sind in Gelb/Rottönen kodiert; Hirnregionen, die während der rechtshändigen Fingerbewegung stärker aktivieren, in Grün-/Blautönen. A: SMC und kaudales SMA beidseits. B: die kaudalen Putamina sowie der Thalamus beidseits. C: SMC, kaudales SMA, Thalamus und Inselrinde beidseits. D: Cerebellum beidseits. A und B sind Transversalschnitte, $C$ und D Frontalschnitte; die Schnittebenen im Talairach-Koordinatensystem sind in $\mathrm{mm}$ angegeben.

Die Signal-Zeit-Verläufe für die aktivierten Anteile des Putamens unterschieden sich deutlich von denen der Konjunktions-Analyse (s. Abbildungen 13 und 14). Die zeitlichen Abläufe der BOLD-Antworten für die Bewegungen der ipsi- und der kontralateralen Hand waren im Prinzip ähnlich, das Signal für die kontralaterale Bewegung war jedoch signifikant stärker als das für die ipsilaterale Bewegung. Während sich in der Konjunktions-Analyse die Reaktion von rostralem Putamen und rostraler SMA als ähnlich erwiesen, zeigte sich in der Kontrast-Analyse eine Ähnlichkeit der Reaktion von kaudalem Putamen und Thalamus. 


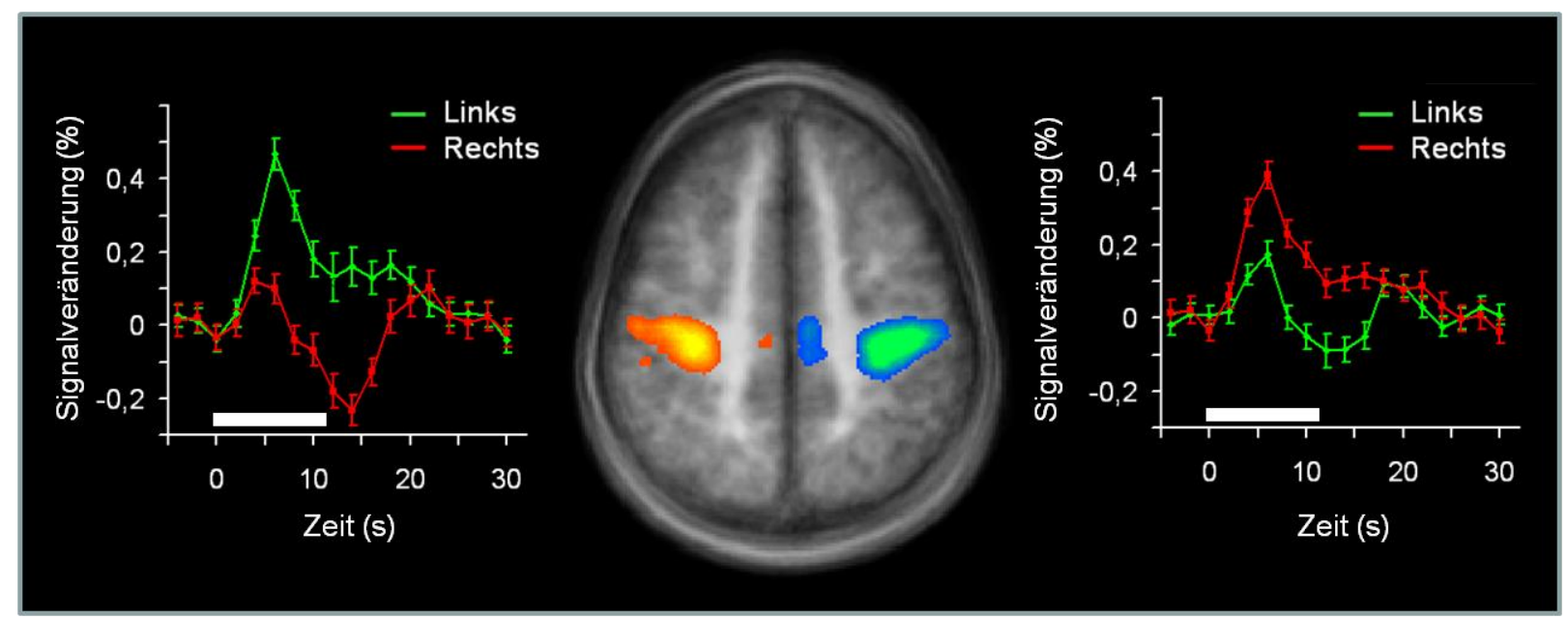

Abbildung 13: Der Primär Sensomotorische Kortex beidseits in der Kontrastanalyse mit Signal-Zeit-Verläufen. Die farblich kodierten Bereiche des SM1 werden funktionell wie ein einziges Voxel betrachtet, dessen Signalveränderung (in \%) in Funktion der Zeit (in s) aufgetragen wird (Signifikanzniveau $p<0,001$; unkorrigiert für multiple Vergleiche). Der weiße Balken markiert die Phase der Stimulation bzw. der Fingerbewegung. Die grüne Kurve stellt den Verlauf für linkshändige, die rote Kurve für rechtshändige Fingerbewegung dar.

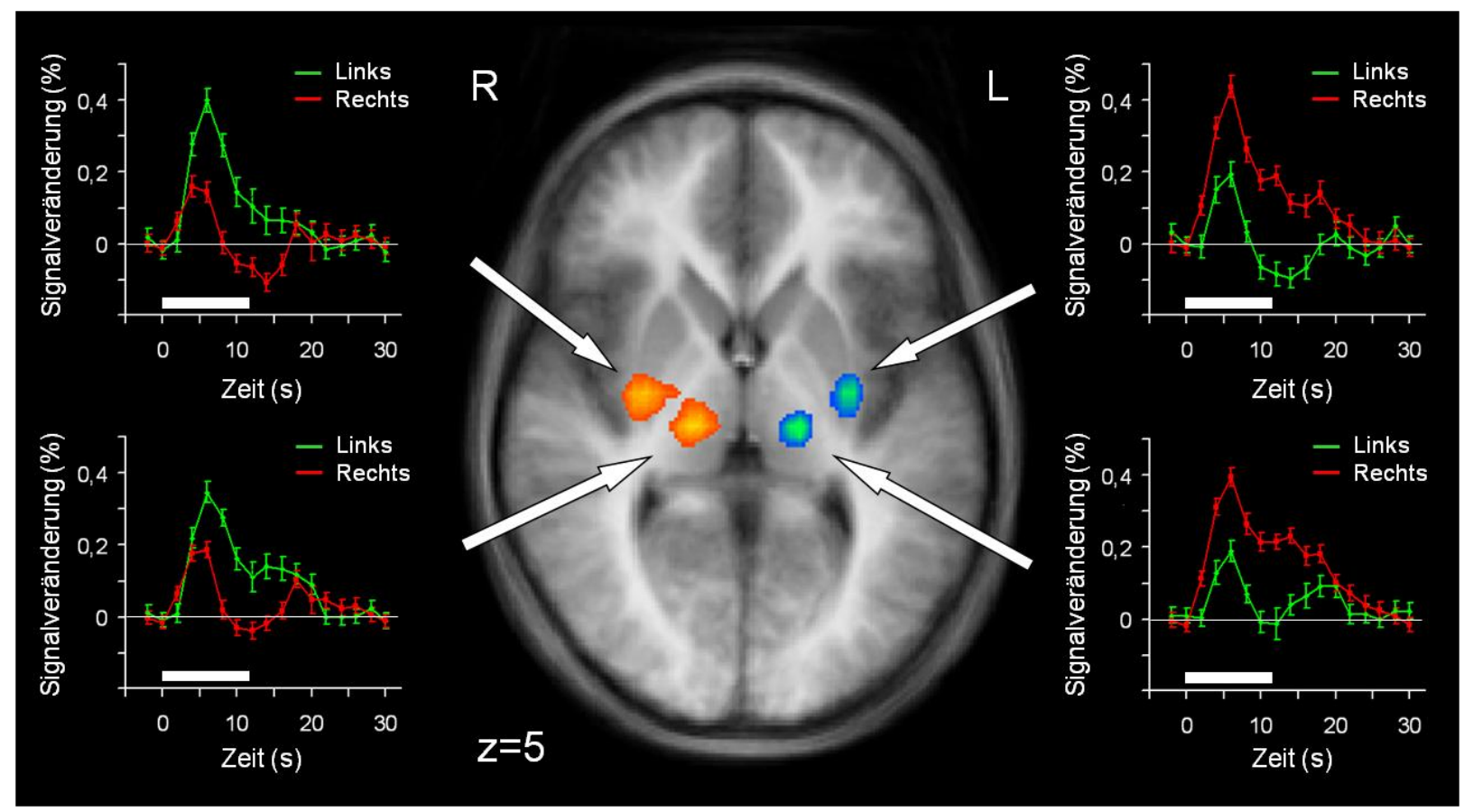

Abbildung 14: Die kaudalen Putamina sowie die Thalami beidseits in der Kontrastanalyse mit Signal-Zeit-Verläufen. Die oberen Signal-Zeit-Verläufe beziehen sich auf die kaudalen Putamina, die unteren auf die Thalami, wie durch die weißen Pfeile markiert. Übrige Angaben wie in Abbildung 13. 
Zusammengefasst lassen sich zwei verschiedene cerebrale Aktivierungsmuster während der einhändigen Fingerbewegung beobachten. Eines der Muster stellt Hirnregionen dar, in denen eine Aktivierung sowohl bei einseitiger Bewegung der linken wie auch der rechten Hand nachweisbar ist. Das zweite Muster hingegen zeigt Regionen auf, die bei der unilateralen Bewegung seitenspezifisch aktiviert sind. In dem SMA und in den Basalganglien können jeweils zwei Subregionen ausgemacht werden, von denen die rostrale das erste Aktivierungsmuster, die kaudale das zweite Aktivierungsmuster aufweist. 


\section{Diskussion}

Im Rahmen unserer Untersuchungen ist es gelungen, zwei kortiko-striato-thalamo-kortikale Schleifen im lebenden menschlichen Gehirn zu identifizieren und zur Abbildung zu bringen. Wir konnten außerdem zeigen, dass es möglich ist, mittels fMRT die sensomotorische und die assoziative Schleife anhand ihrer Lateralitätseigenschaften voneinander zu unterscheiden. Weiterhin sprechen unsere Ergebnisse für die Möglichkeit einer modifizierten, funktionellen Unterteilung des SMA mit einem rostralen, assoziativen und einem kaudalen, sensomotorischen Kompartiment.

\subsection{Das motorische Netzwerk und seine Lateralisierung}

Das motorische Netzwerk, das bei einseitiger Handbewegung involviert ist, war Gegenstand umfangreicher Forschungen und gilt als gut bekannt. Wie erwartet konnte es in unserer Studie klar identifiziert und mithilfe der Aktivierungskarten der Haupteffekt-Analyse visualisiert werden. Hier zeigten sich bereits unterschiedliche Lateralitätseigenschaften verschiedener Hirnareale. SMC und Thalamus waren ausschließlich kontralateral, das Cerebellum ausschließlich ipsilateral zur bewegten Hand aktiviert. SMA, Prä-SMA, ACC, Basalganglien und Insula hingegen zeigten eine bilaterale Beteiligung. Die Berechnung einer Konjunktions-Analyse einerseits und einer Kontrast-Analyse andererseits zeigte, dass bestimmte Hirnregionen in der einseitigen Bewegung sowohl der linken wie auch der rechten Hand involviert sind und demnach keine Seitenspezifität besitzen, während andere Hirnregionen seitenspezifisch bei der Bewegung der kontralateralen Hand aktivieren. Überdies scheinen einige Hirnregionen funktionell unterschiedliche Anteile mit unterschiedlichen Lateralitätseigenschaften zu besitzen.

\subsubsection{Das supplementär motorische Areal (SMA)}

In den Aktivierungskarten der Haupteffekte kam ein ausgedehntes Aktivierungsareal zur Darstellung, das die Regionen SMA, Prä-SMA und ACC beider Hemisphären umfasste. Eine Beteiligung bei der einseitigen Zeigefingerbewegung sowohl der linken wie auch der rechten Hand konnte für rostrale Anteile des SMA sowie für das Prä-SMA nachgewiesen werden. Kaudale Anteile des SMA hingegen zeigten lediglich eine Aktivierung bei Bewegung des 
kontralateralen Zeigefingers und demnach eine seitenspezifische Reaktion (s. Abbildung 15). Die Betrachtung der Signal-Zeit-Verläufe unterstützt diese Beobachtung: Während die BOLDAntworten des rostralen SMA und Prä-SMA für ipsi- und kontralaterale Bewegung nahezu identisch waren, wies das kaudale SMA ein signifikant höheres Signal für kontralaterale als für ipsilaterale Bewegung auf.

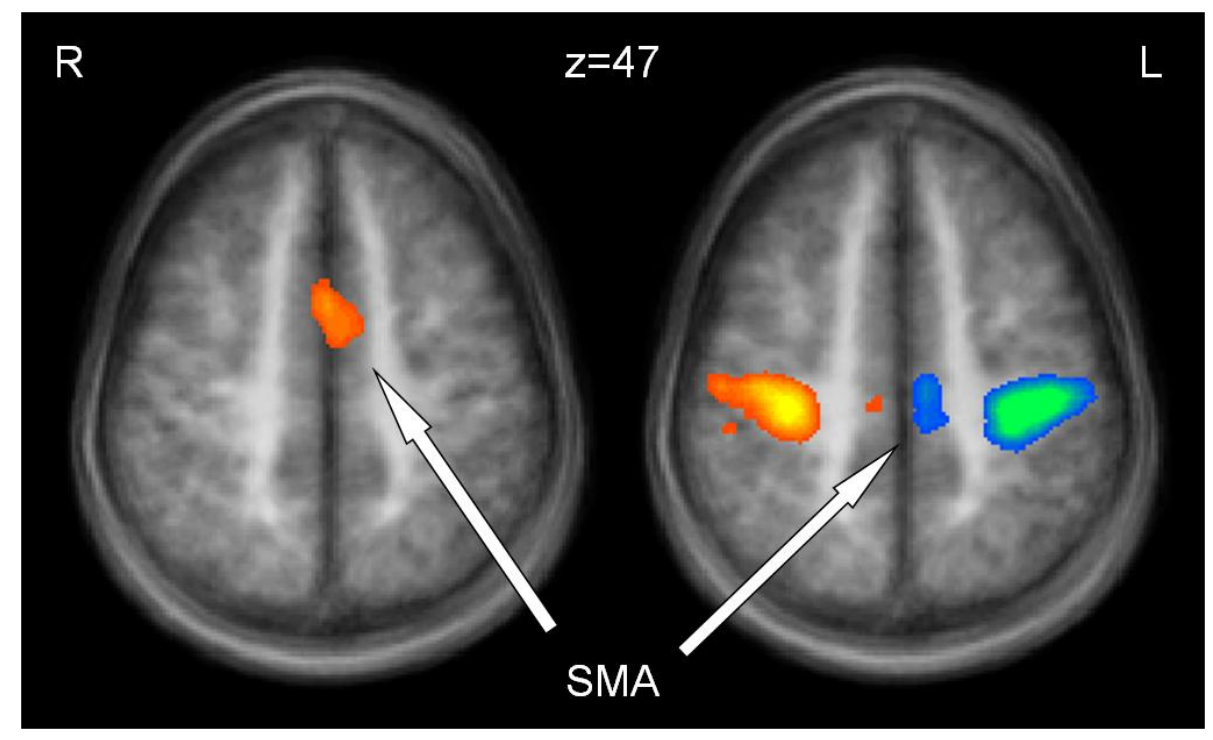

Abbildung 15: Das supplementär-motorische Areal in der Konjunktions- (links) und in der Kontrastanalyse (rechts). Die rostrale SMA ist sowohl während links- als auch während rechtshändiger Fingerbewegung aktiviert; das Signal ist bihemisphäriell. Die kaudale SMA hingegen reagiert seitenspezifisch.

Links: Hirnregionen, die sowohl während der links- als auch während der rechtshändigen Fingergewegung aktiv sind, sind in Gelb-/Rottönen kodiert. Rechts: Hirnregionen, die während der linkshändigen Fingerbewegung stärker aktivieren, sind in Gelb-/Rottönen kodiert; Hirnregionen, die während der rechtshändigen Fingerbewegung stärker aktivieren, in Grün/Blautönen.

Signifikanzniveau $\mathrm{p}<0,001$. Transversalschnitt; die Schnittebene im TalairachKoordinatensystem ist in mm angegeben. $R=$ rechts, $L=$ links.

Befunde vorangegangener Studien bieten eine Grundlage für diese Beobachtungen. Boecker et al. untersuchten mediale motorische Areale mittels PET und fanden, dass der regionale Blutfluss im Prä-SMA von der Komplexität einer motorischen Aufgabe abhängig war, während der Blutfluss im SMA oder im SMC auch über eine Modifikation der motorischen Aufgabe hinweg stabil blieb (Boecker et al. 1998). Eine rostrokaudale Differenzierung kann demnach vermutet werden. Gestützt wird diese Vermutung durch anatomische Studien, aus denen bekannt ist, dass 
das rostrale Striatum bilaterale Verbindungen aus der SMA erhält, während das kaudale Striatum hauptsächlich unilaterale Afferenzen aus dem SMC empfängt (Wiesendanger et al. 1996; Wiesendanger et al. 2004). Auch konnte von Gérardin et al. in einer fMRT-Studie nachvollzogen werden, dass zur bewegten Körperseite ipsilateral gelegene kortiko-striatale Projektionen anterior, also rostral, zu kontralateralen Projektionen verlaufen (Gerardin et al. 2003).

Die Grenze zwischen SMA und Prä-SMA wird gemeinhin bei $\mathrm{y}=0$ nach Talairach angegeben (Picard und Strick 1996; Picard und Strick 2001; Lehericy et al. 2004a). Auf den ersten Blick scheinen die Aktivierungszonen in der vorliegenden Arbeit mit dieser gängigen Einteilung nicht in Einklang zu bringen zu sein. Tatsächlich legen unsere Daten eine weitere Unterteilung des SMA in einen rostralen und einen kaudalen Anteil nahe. Der rostrale Anteil wäre demnach bilateral organisiert und Teil der assoziativen Schleife. Der kaudale Anteil hingegen wäre Teil der sensomotorischen Schleife und unilateral organisiert.

Hinweise für eine solche Einteilung wurden erstmals durch Stephan et al. geliefert, die Handund Armbewegungen in einer PET-Studie untersuchten (Stephan et al. 1995). Sie fanden, dass kaudale Anteile des SMA nur während der tatsächlichen Ausführung einer motorischen Aufgabe aktiviert wurden, rostrale Anteile hingegen auch während der mentalen Vorstellung der Aufgabe. Anatomische Daten unterstützen diese Befunde: Vorobiev et al. wiesen cytoarchitektonische Unterschiede zwischen einem rostralen und einem kaudalen Anteil des SMA nach, wobei sie die beiden Anteile durch eine zur posterioren Kommissur vertikale Linie (VPC-line) trennten (Vorobiev et al. 1998). Sie postulierten eine funktionelle Unterteilung in ein rostrales und ein kaudales SMA und prägten die Bezeichnungen SMAr und SMAc für die jeweiligen Kompartimente. Hierbei bezogen sie sich auf vorangegangene Studien, in denen entsprechende Muster beim Vergleich von mentaler Vorstellung einer motorischen Aufgabe mit der tatsächlichen Ausführung beschrieben worden waren (Tyszka et al. 1994; Roth et al. 1996). Unsere eigenen Daten stimmen mit dem vorgeschlagenen Modell von Vorobiev et al. gut überein, insbesondere da die Grenze zwischen SMAr und SMAc mit der VPC-line exakt kongruent ist. 


\subsubsection{Das Putamen}

Die Haupteffekt-Analyse zeigte eine Signalanhebung des zur bewegten Hand kontralateralen Putamen in seiner gesamten rostrokaudalen Ausdehnung, außerdem des rostralen Anteils des ipsilateralen Putamen. Die Konjunktions-Analyse bestätigte die bilaterale Beteiligung des Putamen bei der unilateralen Handbewegung. In der Kontrast-Analyse hingegen wurde eine signifikante Seitenspezifität des kaudalen Putamen deutlich (s. Abbildung 16). Die Signalzeitverläufe stellten sich für Putamen und SMA ähnlich dar: die rostralen Anteile beider Strukturen zeigten nahezu identische BOLD-Antworten, während die kaudalen Anteile ipsilateral eine signifikant schwächere Reaktion zeigten. Auch für das Putamen sprechen die Befunde demnach für eine funktionelle Unterteilung in ein rostrales assoziatives und ein kaudales sensomotorisches Kompartiment.

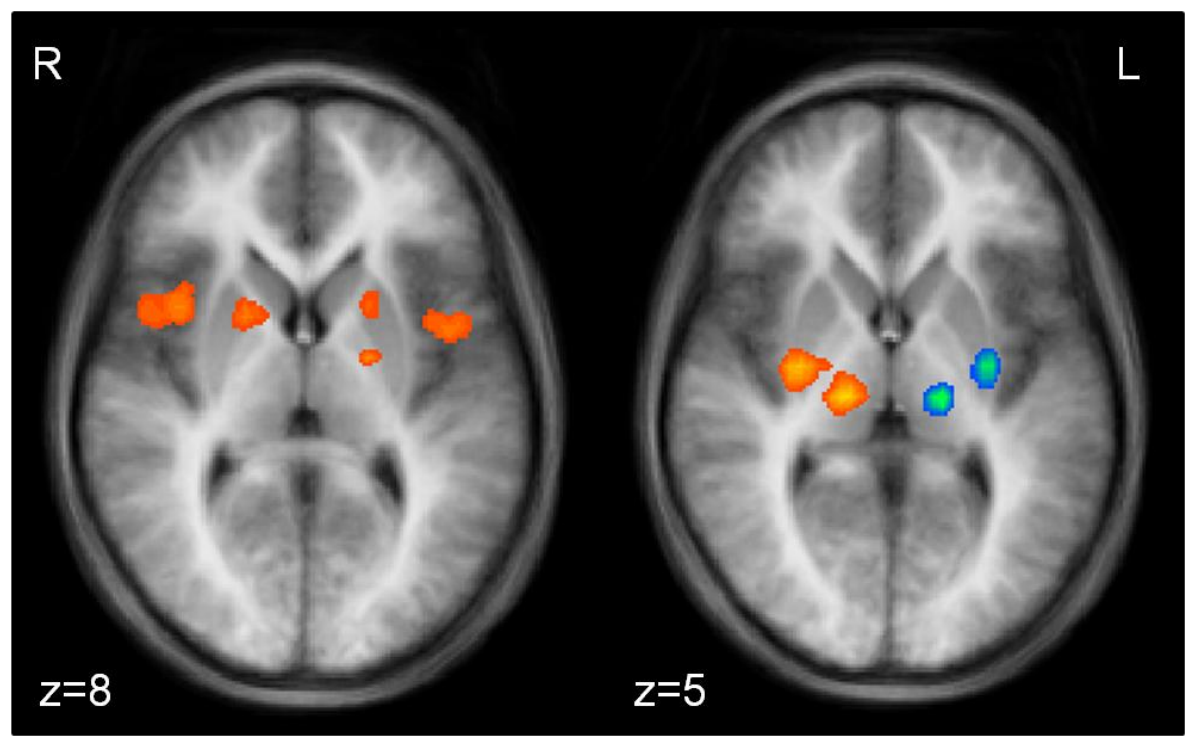

Abbildung 16: Das Putamen in der Konjunktions- (links) und in der Kontrastanalyse (rechts). Rostrale Anteile des Putamen sind sowohl während links- als auch während rechtshändiger Fingerbewegung aktiviert. Kaudale Anteile des Putamen hingegen reagieren seitenspezifisch.

Links: Hirnregionen, die sowohl während der links- als auch während der rechtshändigen Fingergewegung aktiv sind, sind in Gelb-/Rottönen kodiert. Rechts: Hirnregionen, die während der linkshändigen Fingerbewegung stärker aktivieren, sind in Gelb-/Rottönen kodiert; Hirnregionen, die während der rechtshändigen Fingerbewegung stärker aktivieren, in Grün/Blautönen.

Signifikanzniveau $p<0,001$, korrigiert für multiple Vergleiche. Transversalschnitte; die Schnittebenen im Talairach-Koordinatensystem sind in $\mathrm{mm}$ angegeben. $\mathrm{R}=$ rechts, $\mathrm{L}=$ links. 
Jueptner und Weiller konnten in einer PET-Studie zeigen, dass das Erlernen unbekannter motorischer Sequenzen das rostrale Striatum und den DLPFC, also assoziative Hirnregionen, involviert. Bereits bekannte motorische Aufgaben hingegen riefen Aktivierung im kaudalen Striatum hervor (Jueptner und Weiller 1998). Ähnliche Befunde ergab eine fMRT-Studie, die die Unterschiede der striatalen Aktivierung bei der Planung und Durchführung von entweder selbst erdachten oder vorher erlernten motorischen Sequenzen untersuchte (Jankowski et al. 2009). Letztere gingen mit Aktivierung kaudaler striataler Anteile einher, selbst erdachte Sequenzen hingegen mit Aktivierung im assoziativen rostralen Striatum; die Autoren betonen hier eine beidseitige Beteiligung. Lehéricy et al. demonstrierten eine weitere funktionelle Subspezialisierung des menschlichen Striatums, indem sie verschiedene Parameter einer motorischen Aufgabe variierten: sie vermuteten, dass die Frequenz einer Fingerbewegung die Aktivierung im kaudalen Putamen beeinflussen würde, während die Komplexität einer Aufgabe Auswirkungen auf die rostrale Aktivierung habe (Lehericy et al. 2006). Tatsächlich wurde eine Kovariation von SMC und kaudalem Striatum einerseits sowie von prämotorischen Regionen und anteriorem Striatum andererseits beobachtet. Wie für die kortikalen Regionen SMA/Prä-SMA wurde auch für das Striatum die Grenze zwischen assoziativem und sensomotorischem Areal bei $\mathrm{y}=0$ (VAC-Level) angesetzt (Parent 1990; Postuma und Dagher 2006). 


\subsubsection{Das Cerebellum}

Auch Aktivierung im Kleinhirn kann in ein- und beidseitige Beteiligung eingeteilt werden. Bilaterale Beteiligung fanden wir im Vermis, während die Kleinhirnhemisphären seitenspezifisch involviert waren, und zwar, im Gegensatz zum Großhirn, ipsilateral zur bewegten Hand (s. Abbildung 17).

In früheren Studien war Aktivierung im Vermis bereits mit assoziativen Leistungen in Verbindung gebracht worden, Aktivierung in den Hemisphären hingegen mit rein exekutivmotorischen Aufgaben (Sadato et al. 1996a; Kraft et al. 2007). Wenngleich das Kleinhirn in fMRT-Studien kaum erwähnt wird, beschreiben Postuma und Dagher eine Mitaktivierung cerebellärer Anteile im Rahmen der motorischen Schleifen (Postuma und Dagher 2006).

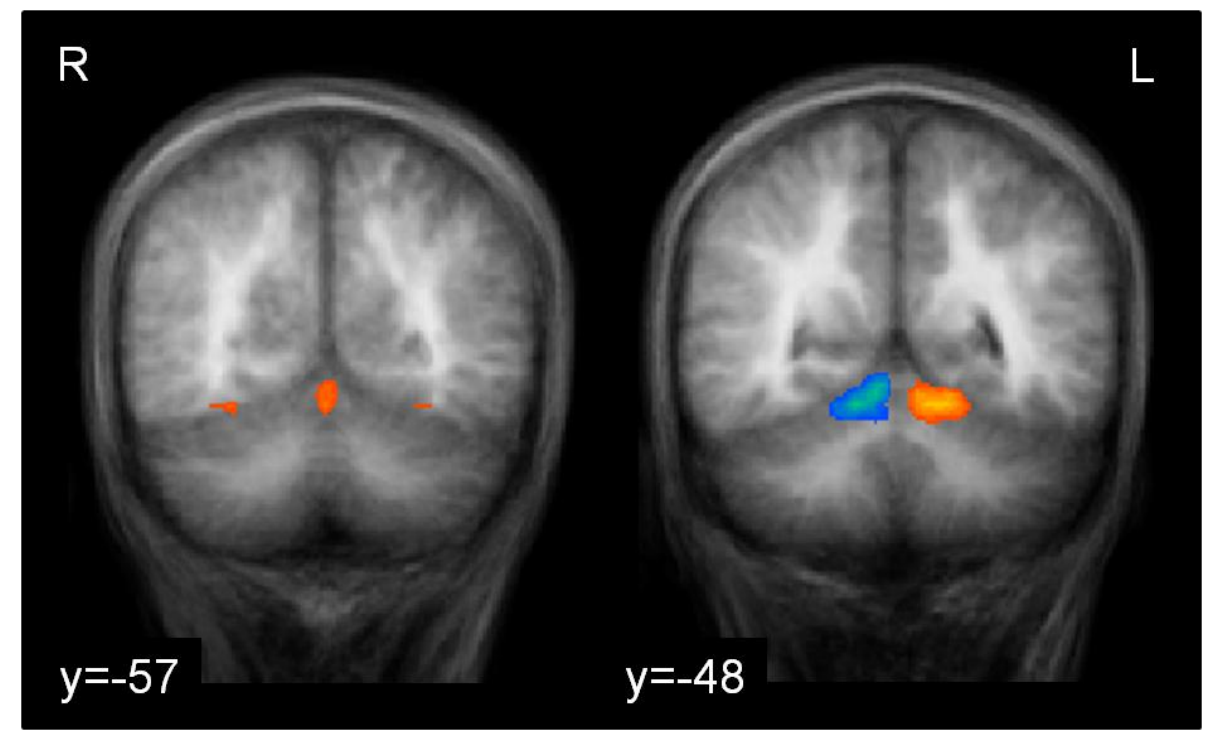

Abbildung 17: Das Cerebellum in der Konjunktions- (links) und in der Kontrastanalyse (rechts). Mediale Anteile des Cerebellums sind sowohl während links- als auch während rechtshändiger Fingerbewegung aktiviert. Laterale Anteile des Cerebellums hingegen reagieren seitenspezifisch.

Links: Hirnregionen, die sowohl während der links- als auch während der rechtshändigen Fingergewegung aktiv sind, sind in Gelb-/Rottönen kodiert. Rechts: Hirnregionen, die während der linkshändigen Fingerbewegung stärker aktivieren, sind in Gelb-/Rottönen kodiert; Hirnregionen, die während der rechtshändigen Fingerbewegung stärker aktivieren, in Grün/Blautönen.

Signifikanzniveau $\mathrm{p}<0,001$, korrigiert für multiple Vergleiche. Linke Spalte: Sagittalschnitte; rechte Spalte: Frontalschnitte. Die Schnittebenen im Talairach-Koordinatensystem sind in $\mathrm{mm}$ angegeben. $\mathrm{R}=$ rechts, $\mathrm{L}=$ links. 


\subsubsection{Koaktivierung und funktionelle Netzwerke}

Hinweise für eine funktionelle Konnektivität von Hirnregionen wurden von Postuma und Dagher in der bereits erwähnten Metaanalyse geliefert (Postuma und Dagher 2006). Funktionelle Konnektivität wurde definiert als „die Tendenz verschiedener Hirnregionen, simultan aktiviert zu sein“. Die Autoren betonten hierbei, dass dies nicht gezwungenermaßen auf direkten, also monosynaptischen anatomischen Verbindungen beruhen müsse. Aufgrund der Auswahl der in die Metaanalyse aufgenommenen Studien können die beschriebenen Koaktivierungsmuster als aufgabenunabhängig gelten. Sie stimmen nicht nur sehr genau mit den theoretischen Vorhersagen des Modells der Dreiteiligkeit überein, sondern lassen sich auch hervorragend in den Aktivierungsnetzwerken unserer Studie wiedererkennen. Auch Postuma und Dagher schlugen eine funktionelle Unterteilung sowohl von SMA/Prä-SMA wie auch vom Putamen vor in jeweils einen rostralen und einen kaudalen Anteil. Die rostralen Anteile zeigten demnach eine Koaktivierung mit medianen Kleinhirnanteilen, dem DLPFC, der rostralen Insula und dem dorsomedialen Thalamus; Regionen, die bekanntermaßen der assoziativen Schleife angehören. Die kaudalen Anteile hingegen zeigten eine Koaktivierung mit dem SMC, SII und lateralen Kleinhirnanteilen, hier liegt die Zugehörigkeit zur sensomotorischen Schleife nahe. Die von Postuma und Dagher beschriebenen Muster und entsprechend postulierte Netzwerke konnten wir hier erstmals in vivo visualisieren und somit bestätigen (s. Abbildung 18).

Unser Studiendesign war mit dem Ziel entworfen worden, sowohl die sensomotorische (durch die Exekution der Fingerbewegung) als auch die assoziative Schleife (selbst generierter Rhythmus, siehe hierzu (Boecker et al. 1998; Deiber et al. 1999; Kraft et al. 2007)) zu involvieren. Demnach waren BOLD-Antworten sowohl in sensomotorischen wie auch in assoziativen Hirnregionen zu erwarten. Mittels der entstehenden Aktivierungsmuster war es uns möglich, diese beiden kortiko-striato-thalamo-kortikalen Schleifen mit den beteiligten Hirnregionen und -unterregionen abzubilden. Demnach umfasst die sensomotorische Schleife den SMC, das SMAc, das kaudale Putamen, SII, den Thalamus sowie laterale Kleinhirnanteile. Außer den Kleinhirnanteilen befinden sich alle beteiligten Regionen in der zur Handbewegung kontralateralen Hemisphäre. An der assoziativen Schleife hingegen sind Prä-SMA, SMAr, das rostrale Putamen sowie Insula, DLPFC, GP und mediane Kleinhirnanteile beteiligt. Von der nur linksseitigen Aktivierung des GP abgesehen, sind alle anderen Aktivierungen bihemisphäriell und symmetrisch und bilden somit den bilateralen Aufbau dieser Schleife ab. 


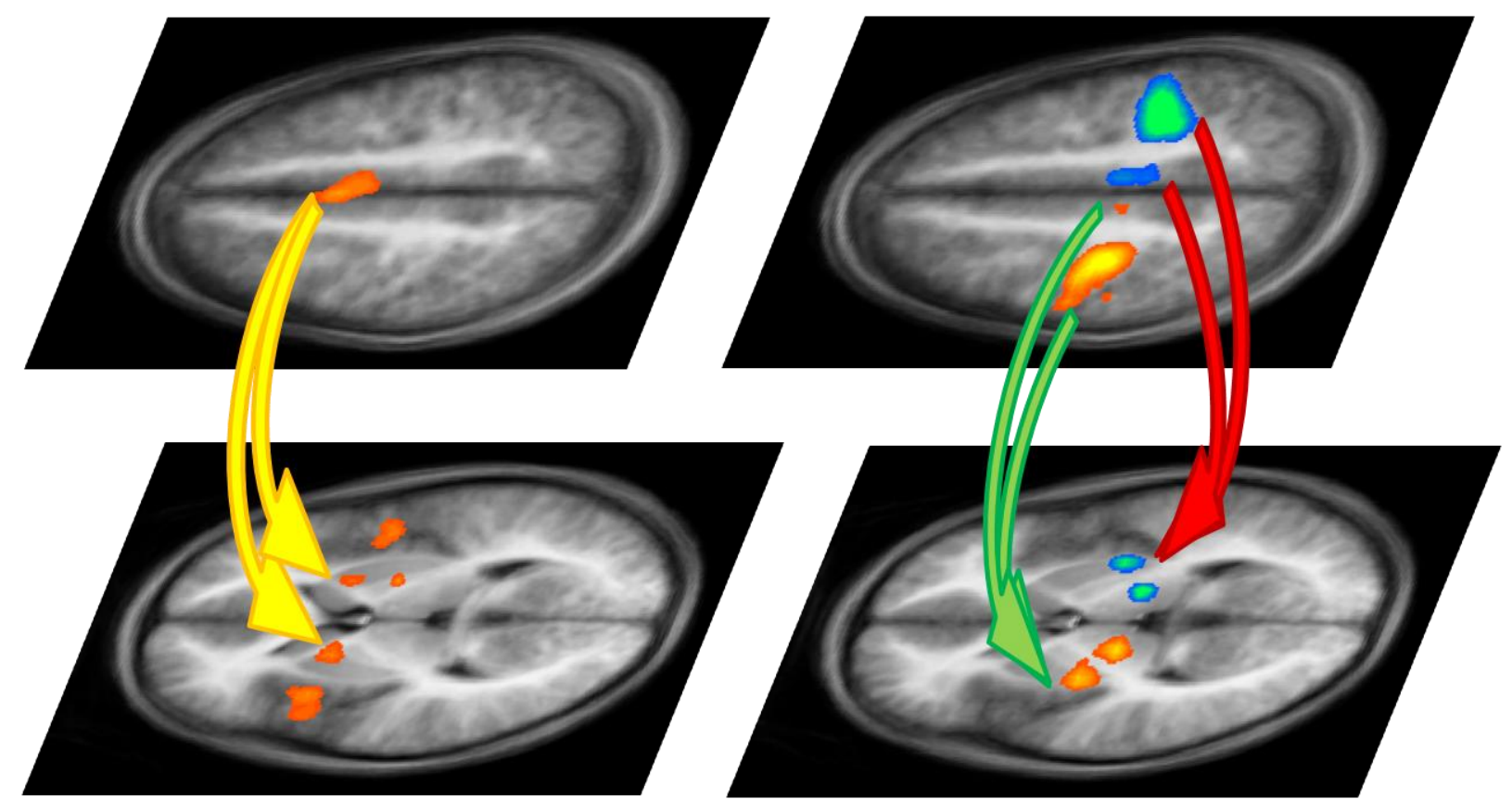

Abbildung 18: Modell für kortikostriatale Projektionen der assoziativen (links) und der sensomotorischen Schleife (rechts). Das SMAr aktiviert bilateral und projiziert ins bilaterale rostrale Putamen (gelbe Pfeile). Der SMC und das SMAc aktivieren kontralateral zur bewegten Körperhälfte und projizieren ins ipsilaterale kaudale Putamen (grüne Pfeile: Aktivität der linken, rote Pfeile: Aktivität der rechten Hand). 


\subsection{Klinische Implikationen}

Viele neurologische Krankheitsbilder gehen mit Bewegungsstörungen einher, so beispielsweise der Morbus Parkinson, die Chorea Huntington, ein ischämischer Apoplex oder eine Hirnblutung. Wenngleich nicht selten definierte Hirnstrukturen als ursächlich für das Störungsbild bekannt sind, so etwa die Substantia nigra beim M. Parkinson oder das Putamen bei der Chorea Huntington, so ist doch immer das motorische Netzwerk in seiner Gesamtheit betroffen. Bildgebungsstudien zeigen pathologische Aktivierungsmuster in diesen Fällen. Methoden der funktionellen Bildgebung wie z.B. die fMRT bieten mit der Möglichkeit der sogenannten Netzwerkanalyse neue Wege der Früherkennung, zur Beobachtung von Krankheitsverlauf und progression sowie zur Evaluation von Therapiestrategien.

\subsubsection{Morbus Parkinson}

Beim Morbus Parkinson ist eine Degeneration dopaminerger, ins Striatum projizierender Neurone der Substantia nigra ursächlich für einen Untergang striataler Neurone. Es resultiert eine Dysregulation des feinabgestimmten Dopaminstoffwechsels. $\mathrm{Zu}$ den typischen motorischen Symptomen Rigor, Tremor und Akinese kommt es erst, wenn etwa 50\% der nigralen und 80\% der striatalen Neurone untergegangen sind. Wenngleich die motorischen Aspekte der Krankheit im Vordergrund stehen, gibt es eine Vielzahl anderer Symptome, die teilweise vorausgehen. Obwohl die Ursache der Erkrankung ein streng lokales Geschehen ist, ist beim Morbus Parkinson doch das gesamte motorische Netzwerk beeinträchtigt (DeLong und Wichmann 2007). Bildgebungsstudien konnten zeigen, dass die kortiko-striato-thalamo-kortikalen Schleifen schon lange vor Auftreten motorischer Symptome verändert sind (Tang et al. 2010). Beim Morbus Parkinson ist aus übereinstimmenden PET-Studien ein charakteristisches striatales Aktivierungsmuster bekannt, das sogenannte Parkinson-Disease Related Pattern (PDRP) (Asanuma et al. 2005; Asanuma et al. 2006) (s. Abbildung 19). Es zeigt einen Hypermetabolismus in Bereichen des Globus pallidus, des Thalamus, der Pons, des Cerebellums und des sensomotorischen Kortex, sowie einen Hypometabolismus in prämotorischen und posterior parietalen Arealen. 


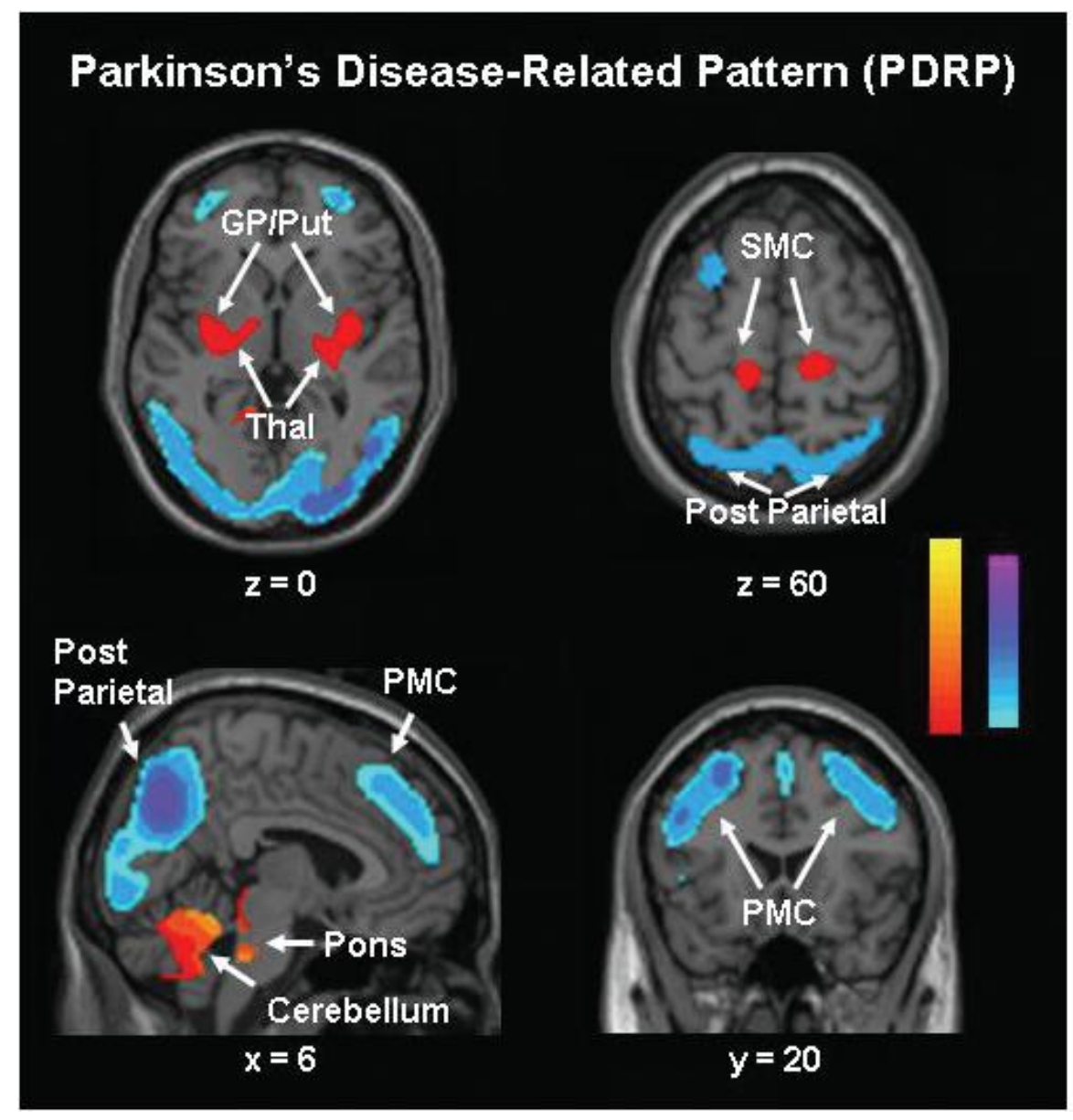

Abbildung 19: Die beim Morbus Parkinson charakteristischen Veränderungen im Aktivierungsmuster (Asanuma et al. 2005, Seite 2670). In einer PET-Studie wurden 20 Patienten mit Morbus Parkinson und 20 gematchte, gesunde Kontrollprobanden untersucht. Vermehrte Stoffwechselaktivität (rot/orange markiert) fand sich im Pallidum, im Thalamus, in Pons und Cerebellum sowie im sensomotorischen Kortex. Verminderte Aktivität (blau markiert) wurde im lateralen prämotorischen Kortex sowie in parieto-okzipitalen Assoziationsarealen gefunden.

Signifikanzniveau $\mathrm{p}<0,001$. GP $=$ Globus pallidus; Put $=$ Putamen; Thal $=$ Thalamus; $\mathrm{SMC}=$ sensomotorischer Kortex; $\mathrm{PMC}=$ prämotorischer Kortex.

Im Striatum konnte in PET- und SPECT-Studien ein asymmetrisches Bild mit rostrokaudalem Gradienten gefunden werden (s. Abbildung 20). Während die Traceraufnahme beim gesunden Probanden seitengleich ist und sowohl im Nucleus caudatus als auch im gesamten Putamen gleichermaßen erfolgt, ist die Aufnahme des Tracers beim Parkinsonpatienten sowohl im rechten gegenüber dem linken Striatum als auch in den kaudalen gegenüber den rostralen Anteilen abgeschwächt (Nandhagopal et al. 2008; Stoessl 2011). 


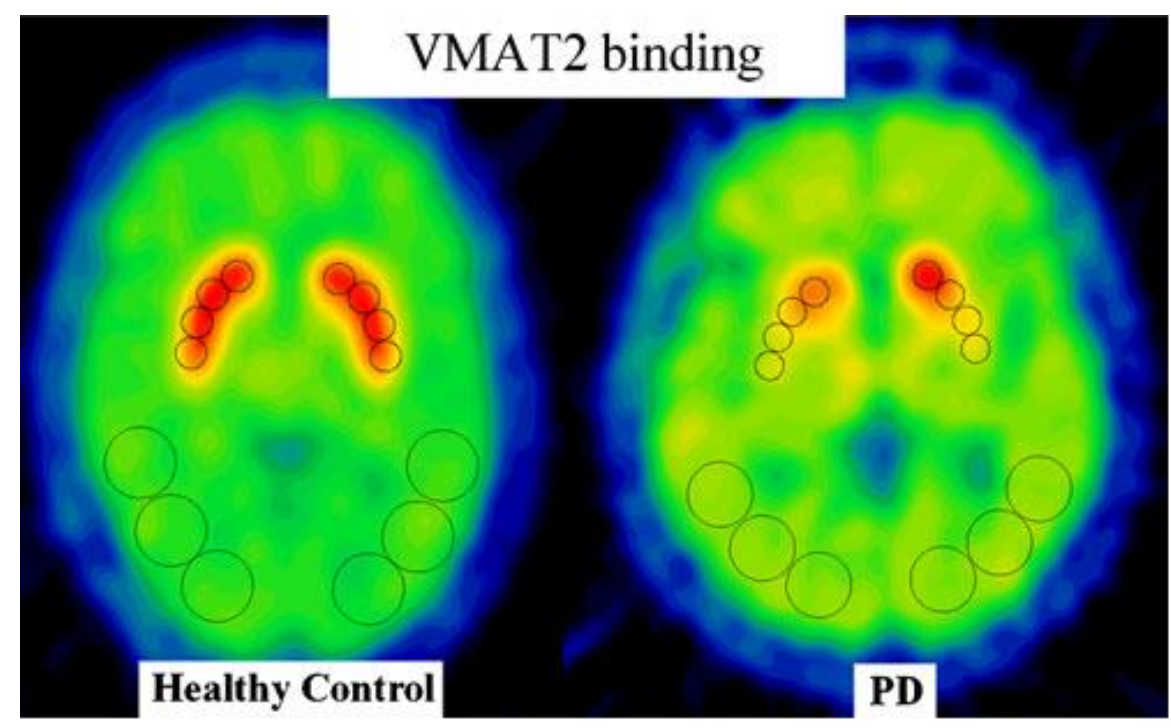

Abbildung 20: Vergleich eines Patienten mit einem mittelschwer ausgeprägten Morbus Parkinson (rechts) und einem gesunden Kontrollprobanden (links); PET-Studie von Stoessl et al. (Stoessl et al. 2011, Seite 75). Man beachte die beim Patienten asymmetrisch reduzierte Traceraufnahme im Putamen bei relativer Bewahrung im Nucleus caudatus.

Die Kreise markieren für die Statistik der Studienautoren entscheidende Regionen. VMAT = vesikulärer Monoamin-Transporter Typ 2; PD = Morbus Parkinson.

Bei familiär vorbelasteten Personen oder in Verdachtsfällen ohne bisher eindeutige Klinik ist das Monitoring während dieser präklinischen Phase bedeutend, denn die neuronalen Netzwerke sind in dieser Phase bereits verändert (Nandhagopal et al. 2008; Tang et al. 2010). Die funktionelle Bildgebung kann hier zur Früherkennung und somit zum frühzeitigen Therapiebeginn beitragen. Weiter bietet sie neben der klinischen Verlaufsbeobachtung eine Methode, Therapiestrategien langfristig auf ihre Wirkung hin zu evaluieren. Tatsächlich konnten Asanuma et al. in einer PET-Studie zeigen, dass das PDRP nach intravenöser Gabe von Levodopa nur noch abgeschwächt nachzuweisen war, und dass die beobachteten Veränderungen des cerebralen Netzwerkes mit einer klinischen Verbesserung der Symptomatik einhergingen (Asanuma et al. 2006). Auch im Hinblick auf sich in der Entwicklung befindliche Verfahren der Neuroprotektion kann eine solche nicht-invasive Möglichkeit der Verlaufsbeobachtung interessant sein (Nandhagopal et al. 2008).

Eine weitere Anwendung wäre das Monitoring nach Zelltransplantation. Die Transplantation von fetalem Gewebe aus dem ventralen Mesencephalon ist derzeit ein experimenteller Ansatz zur reparativen Behandlung des Morbus Parkinson. Nach dem Eingriff sind engmaschige Verlaufskontrollen notwendig, um das Einwachsen des Gewebes, seinen Einfluss auf das motorische Netzwerk und natürlich das klinische Outcome zu überwachen. Politis (Politis 2011) 
beschreibt in einer aktuellen Arbeit die Rolle der funktionellen Bildgebung in der PostTransplantations-Nachsorge. Er fordert, vor allem im Hinblick auf die aktuelle, europaweite Multicenterstudie Transeuro (www.transeuro.org.uk), zu diesem Zwecke optimierte Protokolle für die funktionelle Bildgebung.

Diese Befunde werfen die Frage auf, ob die in der vorliegenden Arbeit beschriebenen motorischen Schleifen beim Morbus Parkinson in unterschiedlicher Weise betroffen sind. Da die bekannten Aktivierungsmuster bei Erkrankten insbesondere in den kaudalen Anteilen des Striatums zu lokalisieren sind, wäre denkbar, dass die exekutive, durch die kaudalen Anteile des Putamens verlaufende Schleife stärker betroffen wäre als die assoziative, rostraler verlaufende Schleife. Dies insbesondere, da der Erkrankte typischerweise Schwierigkeiten mit der konkreten Ausführung einer Bewegung hat, weniger mit ihrer inhaltlichen Planung, z.B. bei der Initiation einer gewohnten Bewegung. Andererseits ist bekannt, dass ein äußerer Taktgeber oder eine Aufforderung die Initiation erleichtert - dies spricht für eine Mitbeteiligung der assoziativen Schleife. Eine Untersuchung von Erkrankten mit der in dieser Arbeit vorgeschlagenen Methode könnte bei dieser Fragestellung hilfreich sein. Weiter könnte die beschriebene Asymmetrie auf einfache und aussagekräftige Weise untersucht werden.

\subsubsection{Chorea Huntington}

Die Chorea Huntington ist eine autosomal dominant vererbte neurodegenerative Erkrankung, die sich - nach anfänglicher Symptomfreiheit - meist während der 3.-5. Lebensdekade manifestiert. Sie ist charakterisiert durch im Verlauf zunehmende unwillkürliche Bewegungen und kognitive sowie psychiatrische Auffälligkeiten.

Auch bei der Chorea Huntington ist die funktionelle Bildgebung ein etabliertes Verfahren zur Untersuchung der cerebralen Funktionsstörung (für eine Übersicht siehe (Kloppel et al. 2009; Paulsen 2009). Aus PET-Studien ist ein charakteristisch verändertes cerebrales Netzwerk bekannt, das Huntington-Disease Related Pattern (HDRP) (Feigin et al. 2001; Feigin et al. 2007). Es zeigt sich ein Hypermetabolismus in Anteilen von Thalamus, Cerebellum, Motorkortex und Okzipitalkortex, sowie ein Hypometabolismus in Anteilen des Striatums und des cingulären Kortex (s. Abbildung 21). Dieses Muster ist bei genetisch Betroffenen schon in der präklinischen, also noch symptomfreien Phase nachzuweisen. In einer Longitudinalstudie (Feigin et al. 2007) wurde interessanterweise deutlich, dass die Veränderungen in der präklinischen Phase zunächst zunehmen, mit näher rückendem Symptombeginn jedoch wieder abnahmen. Dieses Phänomen wird mit Kompensationsmechanismen erklärt, deren Versagen 
den Beginn der motorischen Symptome schließlich bedingt. Die Autoren vermuten den Thalamus als eine für die Kompensation kritische Struktur.

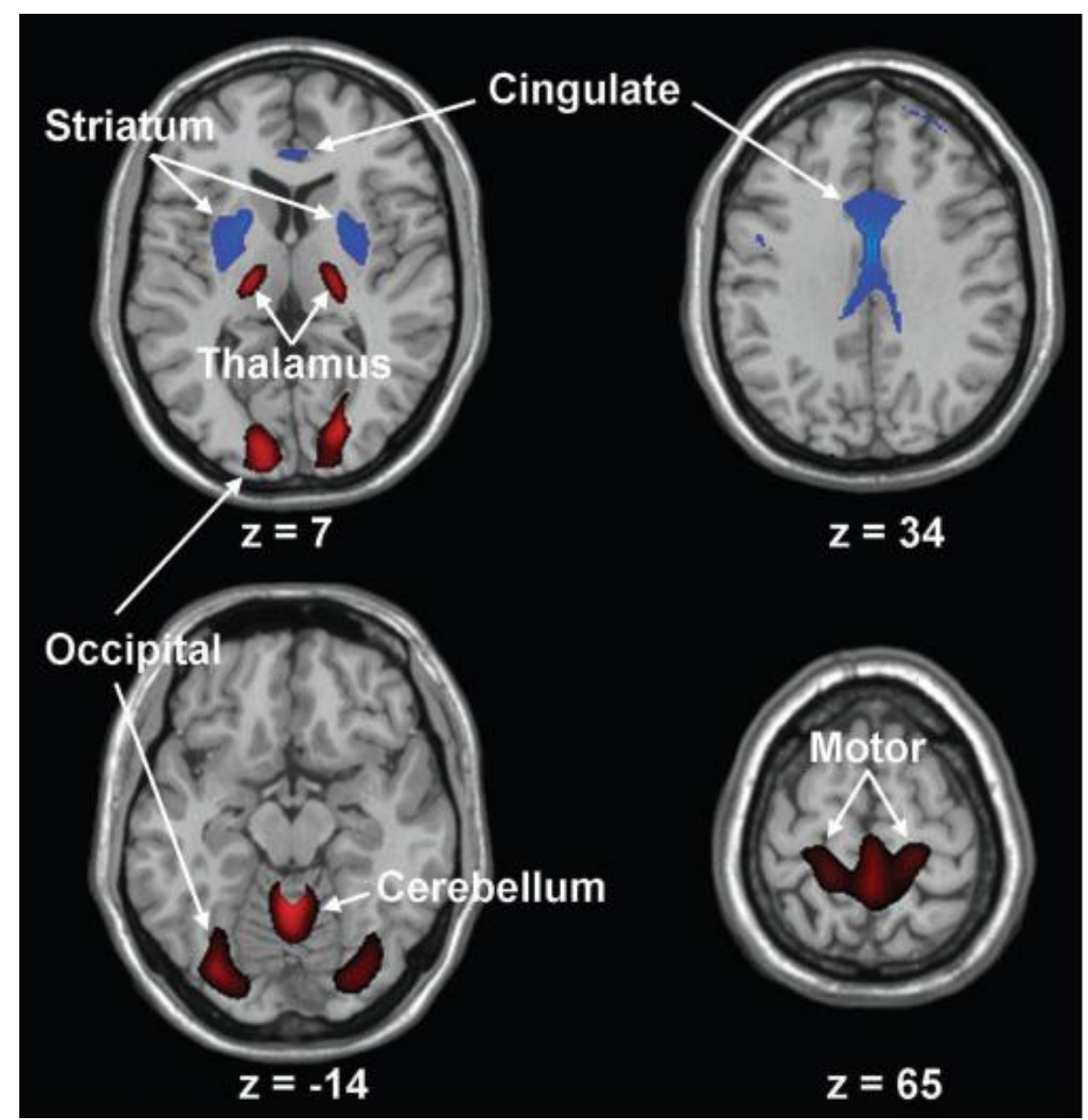

Abbildung 21: Die beim Morbus Huntington charakteristischen Veränderungen im Aktivierungsmuster. Feigin et al. untersuchten mittels PET 12 Patienten mit Morbus Huntington und 12 gematchte, gesunde Kontrollprobanden (Feigin et al., 2007, Seite 2861). Es zeigte sich eine vermehrte Stoffwechselaktivität (rot markiert) im ventralen Thalamus, im Motorkortex und im Okzipitallappen, sowie eine verminderte Aktivität (blau) im Striatum und im Zingulären Kortex. Signifikanzniveau $p<0,001$.

Gerade am Beispiel der Chorea Huntington wird deutlich, wie wichtig eine Methode zur Früherkennung und zur Abschätzung des Krankheitsbeginns ist. Bei positiver Familienanamnese und heutzutage leicht zugänglichen genetischen Untersuchungen kann die Diagnose in vielen Fällen schon früh gestellt werden. Auf diese Weise entsteht ein Zeitfenster bis zum Symptombeginn, das zur Erforschung der präklinischen Phase einerseits und für den Versuch einer Prävention oder zumindest eines Herauszögern des Krankheitsausbruchs genutzt werden kann. Neben klinischen, psychologischen und biochemischen Methoden kann die funktionelle 
Bildgebung als Biomarker dienen, um den frühest möglichen Zeitpunkt für einen - bisher rein symptomatischen - Therapiebeginn $\mathrm{zu}$ definieren, sowie um ihre Wirksamkeit zu evaluieren (Kloppel et al. 2009; Weir et al. 2011). In der Entwicklung neuroprotektiver Maßnahmen kann die fMRT somit als Werkzeug zur Beurteilung von klinischem Verlauf und Therapieeffekt dienen.

\subsubsection{Schlaganfall}

Ein ischämischer Apoplex oder Schlaganfall ist eine akute Hirnschädigung, häufig ausgelöst durch einen Verschluss der Arteria cerebri media. Es resultieren eine Minderversorgung und eine potentiell irreversible Schädigung der abhängigen Hirnregionen. Je nach Lokalisation des Gefäßverschlusses können sowohl die graue Hirnsubstanz kortikal oder subkortikal als auch die weiße Substanz betroffen sein. Typisches Symptom eines Schlaganfallpatienten ist eine eingeschränkte motorische Leistung der zur Läsion kontralateralen oberen Extremität, insbesondere der Handfunktion.

Nach einem ischämischen Ereignis können charakteristische Veränderungen des motorischen Netzwerkes beobachtet werden. Interessant ist der Vergleich von Patienten in der chronischen Phase nach einem Schlaganfall im Vergleich zum Gesunden. Insbesondere die Frage, welche Modifikationen des Netzwerkes das Wiedererlangen motorischer Fähigkeiten begleitet bzw. ermöglicht, wurde in den letzten Jahren eingehend untersucht und beispielsweise von N. Ward in einer Übersichtsarbeit umfassend diskutiert (Ward 2006).

Übereinstimmend wird eine vermehrte Aktivierung sowohl der geschädigten als auch der gesunden Hirnhälfte beschrieben, und zwar in sensomotorischen wie auch in assoziativen kortikalen Arealen. Die vermehrte Rekrutierung assoziativer Areale könnte mit einem erhöhten Planungsaufwand erklärt werden. Eine alternative Erklärung wäre jedoch, dass SMA und andere assoziative kortikale Areale über direkte Projektionen ins Rückenmark die Aufgabe der geschädigten primärmotorischen Bezirke übernehmen.

Die Karten von Bildgebungsstudien mit Apoplex-Patienten zeigen ausgedehnte Aktivierungsareale beidseits sowohl in prämotorischen (z.B. SMA) als auch in primär sensomotorischen Arealen (s. Abbildung 22). Interessant wäre es nun zu untersuchen, ob die in dieser Arbeit vorgestellten motorischen Schleifen gleichermaßen verändert sind. Denkbar wäre, dass hauptsächlich die assoziative Schleife als „planende Institution“ beider Hemisphären vermehrt aktiviert werden muss, und dass die beobachtete Mehraktivierung der gesunden Hirnhälfte über- 
wiegend dadurch bedingt ist. Da aber auch im kontraläsionalen primär sensomotorischen Kortex eine vermehrte Aktivierung beobachtet werden kann, wäre eine differenzierte Betrachtung beider Schleifen, z.B. mittels der in dieser Arbeit vorgeschlagenen Methode, von besonderem Interesse.

Tatsächlich ist eine Veränderung der Lateralitätseigenschaften der motorischen Schleifen nach ischämischem Apoplex beschrieben. Der sogenannte Lateralitätsindex (LI) als Indikator unterschiedlicher Beteiligung der Hirnhälften kann zugunsten der gesunden, aber auch zugunsten der geschädigten Hemisphäre verändert sein. Auch zur weiteren Klärung dieses Phänomens wäre eine Untersuchung mit Differenzierung der motorischen Schleifen interessant.

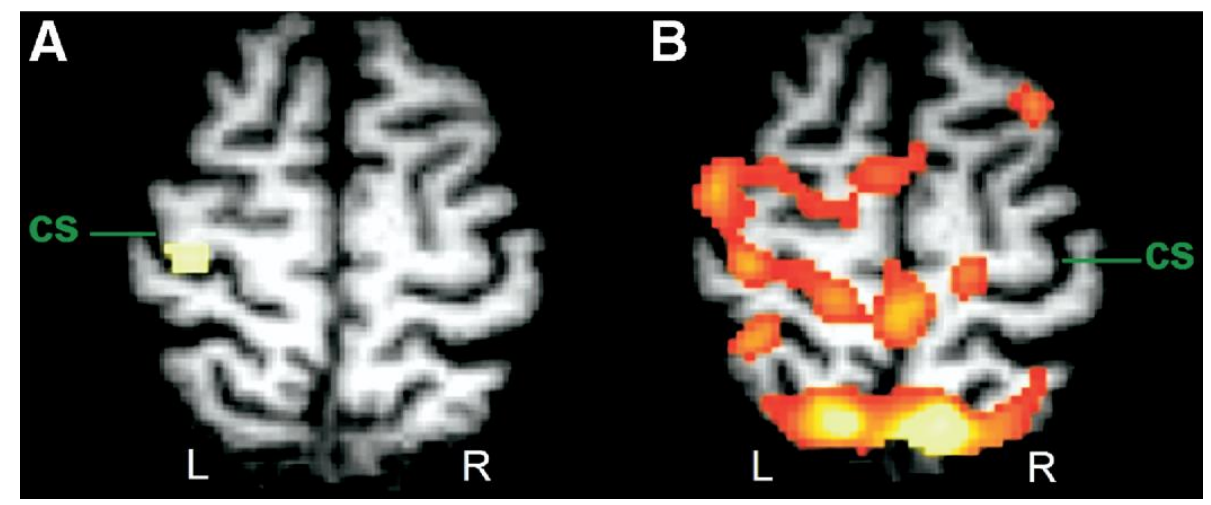

Abbildung 22: Aktivierungskarten eines Schlaganfall-Patienten mit ausgedehnter subkortikaler rechts-hemisphärieller Hirnschädigung 6 Monate nach dem Ereignis. A) Hirnaktivität im SM1 links bei motorischer Aktivität der gesunden rechten Hand. B) Hirnaktivität im SM1 rechts und weiteren Regionen bei motorischer Aktivität der betroffenen linken Hand (Ward 2006, S 33). $R=$ rechts; $L=$ links; $C S=$ Zentralsulcus.

In der Rehabilitation von Schlaganfallpatienten spielen neben physio- und ergotherapeutischen Maßnahmen in den letzten Jahren auch neue Konzepte eine Rolle, wie beispielsweise die „Spiegeltherapie“ oder die „Mentale Vorstellung“. Hierbei können Bildgebungsmethoden wie die fMRT helfen, verschiedene Therapiekonzepte und -erfolge nicht-invasiv zu vergleichen und zu evaluieren. Beim Krankheitsbild des Apoplex bietet die Kombination von fMRT und Transkranieller Magnetstimulation (TMS) die Möglichkeit, die gegenseitigen Einflüsse verschiedener Kortexareale aufeinander $\mathrm{zu}$ untersuchen und $\mathrm{zu}$ modifizieren (z.B. die primär sensomotorischen Kortizes untereinander, aber auch sensomotorische und assoziative Areale). 
In den vorangegangen Abschnitten sind die vielfältigen Anwendungsmöglichkeiten funktioneller Bildgebungsmethoden beschrieben worden. Die fMRT bietet gegenüber der PET die Vorteile der Nicht-Invasivität, der aufgrund fehlender Strahlenbelastung besseren Wiederholbarkeit der Untersuchung sowie eine geringere Kostenintensität.

Mit dem in dieser Arbeit vorgeschlagenen Paradigma lassen sich motorische Komponenten einer Erkrankung auf vergleichsweise einfache Weise untersuchen. Vorteile gegenüber anderen fMRT-Paradigmen sind hierbei insbesondere gegeben durch die Simplizität der motorischen Aufgabe mit stabilen, robusten Ergebnissen. Die inter- sowie die intraindividuelle Vergleichbarkeit im Langzeitverlauf wird erleichtert. Durch die Einbeziehung beider Körperhälften und entsprechend beider Hemisphären kann eine seitendifferente Ausprägung eines Merkmals sowie seines Verlaufs erfasst werden. Über die Differenzierung von assoziativem und exekutivem motorischen Netzwerk können die verschiedenen Komponenten einer Bewegungsstörung sowie der beteiligten Hirnregionen unterschieden werden. Die Einfachheit der Aufgabe ermöglicht die Untersuchung von motorisch oder kognitiv beeinträchtigten Patienten und Probanden.

\subsubsection{Tic-Störung und Tourette-Syndrom}

Eine Tic-Störung ist eine Bewegungsstörung, bei der es zu unwillkürlichen und unkontrollierbaren Muskelzuckungen oder aber zu Lautäußerungen kommt. Treten mehrere verschiedene Tics auf oder sind mehrere Muskelgruppen betroffen, liegt definitionsgemäß ein TouretteSyndrom (TS) vor. Es handelt sich um vergleichsweise häufige Störungsbilder, die meist im Kindes- und Jugendalter auftreten und Jungen häufiger als Mädchen betreffen. In Deutschland werden sie dem kinder- und jugendpsychiatrischen Zuständigkeitsbereich zugeordnet. Eine Behandlung kann neben psychotherapeutischen Maßnahmen auch mit einer medikamentösen Therapie oder, in extremen Fällen, mit Tiefenhirnstimulation erfolgen.

Die Ätiologie des TS ist nicht eindeutig geklärt. Eine familiäre Häufung kann beobachtet werden, was eine zumindest teilweise erbliche Genese nahelegt. Bisher sind jedoch keine Gene identifiziert worden. Auch über die Pathophysiologie beim TS ist wenig bekannt.

Wie eingangs beschrieben, habe ich meine Arbeit im Rahmen eines Forschungsprojektes angefertigt, in dem es um die Erforschung des motorischen Systems einerseits, und um einen Vergleich von Jungen mit TS im Vergleich zu gesunden Kontrollprobanden andererseits ging. Es wurde eine Kohorte von TS-Patienten mit einer Kontrollgruppe verglichen. Zunächst wurde untersucht, ob sich zwischen Patienten und Kontrollprobanden Unterschiede in Morphologie 
und Volumen verschiedener Hirnstrukturen zeigen (Roessner et al. 2010). Tatsächlich konnten größere Volumina der Putamen beidseits gefunden werden, ebenso wie eine größere Subregion des Corpus callosum, die motorische Areale beider Hirnhälften miteinander verknüpft. In einer ersten fMRT-Studie führten Patienten und Kontrollprobanden eine Fingerbewegung mit der dominanten rechten Hand durch (Roessner et al. 2011). Es zeigte sich bei den TS-Patienten eine verminderte Aktivierung des zur Bewegung kontralateralen Gyrus präzentralis und im rechten Parietallappen, sowie eine Signalsteigerung im linken Nucleus caudatus und im rechten medialen Gyrus frontalis. Die beobachteten Auffälligkeiten dieser Strukturen lassen auf ein verändertes motorisches Netzwerk bei TS-Patienten schließen.

Um dieses Netzwerk im Allgemeinen und bei TS-Patienten im Besonderen weiter zu untersuchen und dabei den Limitationen der vorangegangenen Rechnung zu tragen, wurde die fMRTStudie durchgeführt, im Rahmen derer ich die vorliegende Arbeit verfasst habe. Während sich meine Arbeit mit der Untersuchung des motorischen Netzwerkes beim Gesunden beschäftigt, haben wir außerdem Unterschiede zwischen TS-Patienten und Kontrollprobanden gefunden (Roessner et al., im Druck). Das motorische Netzwerk ist bei TS-Patienten im Vergleich zu gesunden Probanden verändert, insbesondere bei der Fingerbewegung der linken, nicht dominanten Hand. Eine verminderte Signalintensität konnte im zur Bewegung kontralateralen SM1 gefunden werden. Die Erklärung hierfür kann eine im Vergleich zur Kontrollgruppe erhöhte Grundaktivität während der Ruhebedingung sein. Eine Signalsteigerung in verschiedenen motorischen Arealen spiegelt ein höheres kortikales Aktivitätsniveau wieder. Dies kann gleich einer vermehrten Anstrengung interpretiert werden, die notwendig ist, um die geforderte Leistung erbringen und Tics während dieser Zeit unterdrücken zu können. In einer kürzlichen Studie zur Feinmotorik zeigten Jungen mit Tourette-Syndrom im Vergleich zu gesunden Kontrollprobanden jedoch vergleichbare Leistungen (Buse et al., 2012).

In einer kürzlich in den USA durchgeführten PET-Studie wurden ebenfalls die metabolischen Netzwerke bei Patienten mit Tourette-Syndrom untersucht (Pourfar et al.). Auch hier konnte ein auf charakteristische Weise verändertes Netzwerk identifiziert werden. Es zeigte sich eine gesteigerte Aktivität im prämotorischen Kortex sowie im Kleinhirn sowie eine reduzierte metabolische Aktivität im Striatum im Orbitofrontalkortex, außerdem (s. Abbildung 23). Neben der reinen Identifikation des veränderten Netzwerkes ist außerdem eine Korrelation zwischen dem Grad der Veränderung und dem klinischen Schweregrad der Symptome festzustellen. Die Autoren weisen auch hier auf die Möglichkeit einer Therapieevaluation hin. Unter einer wirksamen Behandlung sollte die Ausprägung der Netzwerkveränderungen zurückgehen. 
Beim TS bietet die in der vorliegenden Arbeit vorgeschlagene Untersuchungsmethode demnach einen Ansatz zur weiteren Erforschung des Störungsbildes. Beispielsweise wäre die Frage interessant, ob beim TS sowohl die assoziative, planende, als auch die sensomotorische, exekutive Schleife verändert ist, oder ob Veränderungen in einer der Schleifen überwiegen. Weiter ist die Evaluation verschiedener Therapiemaßnahmen denkbar.

\section{A TS-related metabolic pattern}

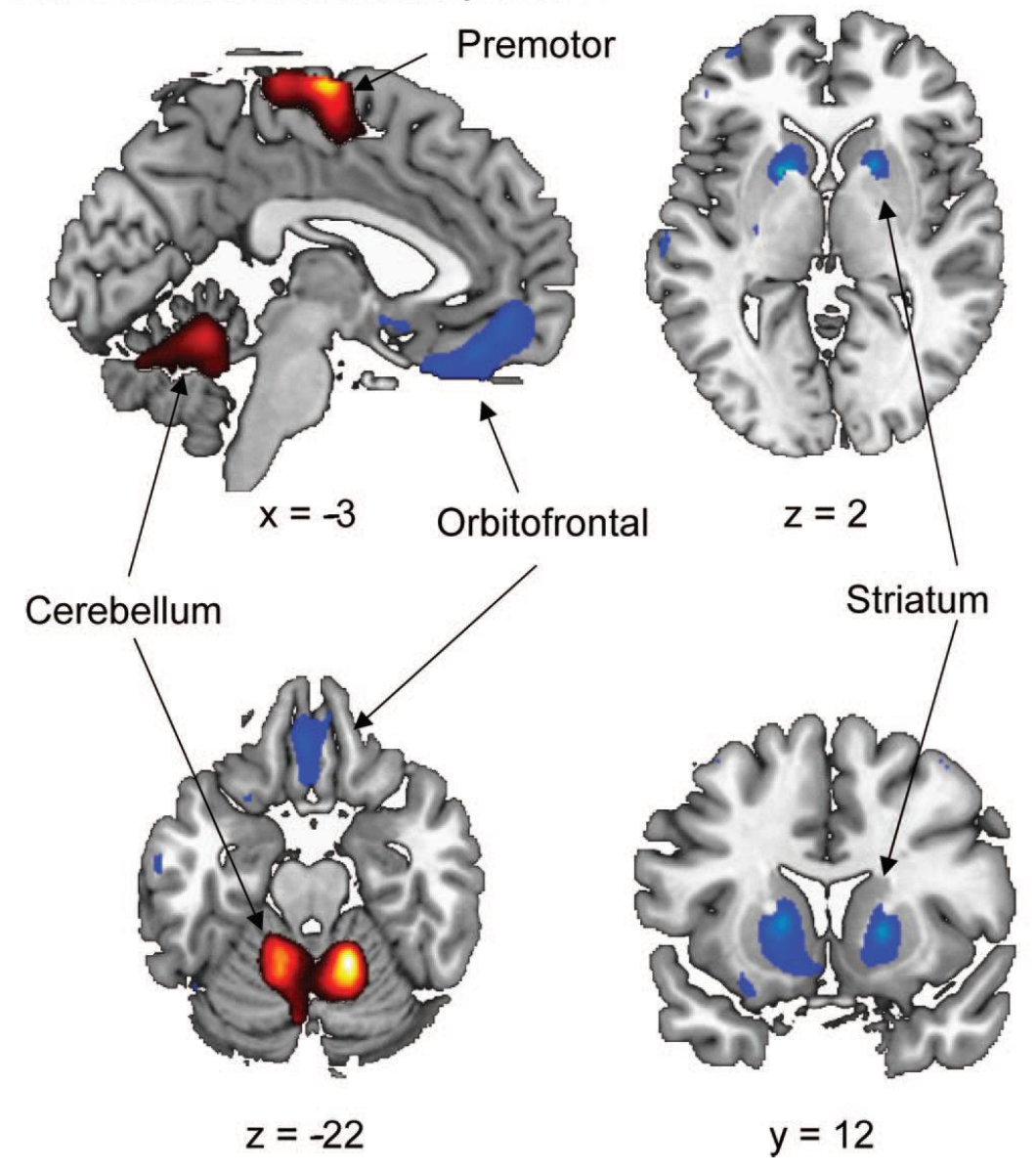

Abbildung 23: Kovarianz-Analyse einer PET-Studie; Vergleich einer Gruppe von TSPatienten mit gesunden Kontrollprobanden, jeweils $n=12$. Bei TS-Patienten besteht eine Aktivitätssteigerung in Prämotorischen Kortexarealen und im Kleinhirn (gelb-rot) sowie eine verminderte Aktivität im Striatum sowie im Orbitofrontalkortex (blau-grün). Die Koordinaten der Ebenen sind in $\mathrm{mm}$ angegeben. 


\subsection{Limitationen}

Nicht alle der bekanntermaßen an den Schleifen beteiligten Hirnregionen konnten in unserer Studie zur Darstellung gebracht werden. Beispielsweise zeigt das GP in der Konjunktionsanalyse nur linksseitig signifikante Werte. Der Thalamus kann nur in der Kontrastanalyse, nicht aber in der Konjunktionsanalyse dargestellt werden. Wir vermuten, dass dies mit der geringen anatomischen Größe der entsprechenden Hirnarealen zu erklären ist. Durch die Koregistrierung der Daten verschiedener Probanden mit individueller Anatomie kommt es gerade bei anatomisch kleinen Strukturen zu einer größeren Varianz.

Die Vergleichbarkeit unserer Ergebnisse mit früheren Studien mag durch die Auswahl unserer Probanden, nämlich Jungen von 10 bis 15 Jahren, eingeschränkt sein. Untersuchungen zeigten jedoch, dass das menschliche Gehirn im Alter von 12 Jahren bereits sowohl bezüglich seiner Größe als auch bezüglich des Gewichts nahezu 100\% der endgültigen Werte erreicht hat (Paus et al. 2001; Lenroot und Giedd 2006; Giedd et al. 2009). Auch ergab ein überprüfender Vergleich unserer Koordinaten im Talairach-Koordinatensystem mit denen aus der Metaanalyse von Postuma und Dagher hochkonsistente Übereinstimmungen. Zwar unterliegen sowohl die graue wie auch die weiße Hirnsubstanz bis ins dritte Lebensjahrzehnt hinein dynamischen Veränderungen, die meisten Fasertrakte sind jedoch bereits in der frühesten Kindheit myelinisiert. Insbesondere basale Hirnfunktionen wie das motorische System gelten als in grauer und weißer Substanz früh ausgereift (Lenroot und Giedd 2006; Toga et al. 2006; Giedd et al. 2009).

Die Symmetrie der Aktivierungsmuster für die dominante rechte und die nicht-dominante linke Hand könnte hingegen tatsächlich ein altersabhängiges Phänomen sein. Bei Erwachsenen könnte eine motorische Leistung der nicht-dominanten Hand eine stärkere und ausgedehntere Rekrutierung von prämotorischen Regionen hervorrufen. Asymmetrische Muster wurden beispielsweise von Haaland et al. beschrieben (Haaland et al. 2004).

Es ist außerdem kritisch zu hinterfragen, ob ein allgemein gültiges Modell des motorischen Systems aus einer Studie lediglich über Fingerbewegung abgeleitet werden kann. Tatsächlich haben vorangegangene Studien - abhängig von der untersuchten Körperpartie - unterschiedliche Lokalisationen von Aktivierungen beschrieben, entsprechend der zugrundeliegenden Somatotopie. Diese somatotopisch bedingten lokalen Unterschiede scheinen jedoch innerhalb einer Hirnstruktur lokalisiert zu sein: die kortiko-subkortikalen Schleifen konnten ebenso bei Aufgaben von Fußbewegungen nachvollzogen werden (Lehericy et al. 1998; Gerardin et al. 2003). Dennoch kann sich dies für komplexere motorische Aufgaben anders verhalten, insbe- 
sondere, wenn die Aufgaben verschiedene Körperpartien involvieren, beispielsweise solche mit weniger offensichtlicher Lateralisierung (Mund, Zunge, Augen). Dies kann Gegenstand zukünftiger Forschung sein, ebenso wie die Frage, ob unsere Hypothese auch für komplexere motorische Aufgaben zutrifft, bei denen weitere Planungsareale wie z.B. der laterale Präfrontalkortex oder parietale Areale involviert sind.

\subsection{Ausblick}

In der Zusammenschau von theoretischem Hintergrund, klinischer Bedeutung und genannten Limitationen ergeben sich Perspektiven zukünftiger Anwendung der in meiner Arbeit vorgestellten Untersuchungsmethode. In der neurowissenschaftlichen Grundlagenforschung kann die hier vorgestellte Differenzierungsmöglichkeit der motorischen Schleifen neue Aspekte liefern. Interessant wäre hier zunächst einmal die Untersuchung gesunder erwachsener Probanden, insbesondere im Hinblick auf die Frage der vollständig ausgeprägten Händigkeit - besteht die Symmetrie der Aktivierungsmuster im Erwachsenenalter fort?

In der klinischen Forschung kann die vorgestellte Untersuchungstechnik Anwendung im Bereich von Diagnostik, Verlaufsbeobachtung und Therapieevaluation finden. Die Differenzierung des motorischen Netzwerks in eine planende, assoziative, und eine rein exekutive, sensomotorische Schleife ermöglicht einen differenzierteren Blick auf die verschiedenen Aspekte einer Bewegungsstörung. Zukünftig wäre die Untersuchung von Patienten mit den in der Arbeit diskutierten Erkrankungen interessant. Denkbar wäre die Möglichkeit einer verfeinerten Darstellung der bei der Krankheit beeinträchtigten Netzwerke. Auch die systematische Evaluation verschiedener Therapieansätze (medikamentös, psychotherapeutisch, physiotherapeutisch) sowie die Wirksamkeit prophylaktischer Maßnahmen kann in der Folge untersucht werden. Interessant wäre weiter, ob eine Früherkennung wenig symptomatischer oder gar asymptomatischer Risikopatienten mithilfe der Identifikation von veränderten motorischen Netzwerken gelingen kann. 


\section{Zusammenfassung}

Der Willkürmotorik liegt ein komplexes neuronales Verschaltungssystem zugrunde, das aus verschiedenen Hirnstrukturen und ihren Verknüpfungen besteht. Ihre Kenntnis ist Voraussetzung für ein Verständnis dafür, wie vage Handlungsideen in konkrete motorische Entwürfe und in präzise Bewegungen umgesetzt werden können. Der Entschluss zu einer Bewegung wird dem limbischen System zugeschrieben. Für die Vorbereitung und Planung einer Bewegung hat der präfrontale Assoziationskortex, u.a. das supplementär motorische Areal (SMA), eine entscheidende Bedeutung. Der letztliche Bewegungsbefehl geht vom primären sensomotorischen Kortex aus (SMC). Hier nimmt der Tractus corticospinalis, die Pyramidenbahn, den Ursprung. Eine Überarbeitung und eine Korrektur des Bewegungsentwurfes erfolgen im Wesentlichen in zwei kortiko-subkortikalen Schleifen, wovon eine über die Basalganglien, die andere über das Kleinhirn verläuft. Im Kleinhirn erfolgt die Feinabstimmung für eine flüssige, koordinierte Bewegung. Die modifizierten Informationen aus Basalganglien und Kleinhirn gelangen in den Thalamus, von wo sie nach einer Integration zurück in die kortikalen motorischen Areale projiziert werden.

Die Basalganglien bestehen aus vier Kernen, dem Striatum, dem Globus pallidus, der Substantia nigra und dem Nucleus subthalamicus. Das Striatum kann seinerseits wiederum in Nucleus caudatus und Putamen unterteilt werden. Die Efferenzen aus dem Kortex gelangen zunächst ins Striatum. Die Informationen verschiedener Kortexareale werden dabei in einer „Parallelschaltung“ unabhängig voneinander prozessiert. Nach einem weitgehend akzeptierten Modell sind Kortex und Striatum und demnach kortiko-striatale Projektionen in drei sowohl anatomisch als auch funktionell getrennte Schleifen unterteilt: die sensomotorische, die assoziative und die limbische Schleife. Ihre Anteile übermitteln Informationen zwischen den jeweiligen kortikalen und subkortikalen Arealen.

Das dorsale Striatum besteht demnach aus zwei Kompartimenten: dem sensomotorischen einerseits und dem assoziativen andererseits, jeweils eingebettet in die entsprechenden Schaltkreise. Kaudolaterale Anteile des Putamens (posterior der anterioren Kommissur, AC) erhalten Afferenzen aus kortikalen sensomotorischen Arealen und können daher als sensomotorisches Kompartiment gelten. Rostromediale Anteile (anterior der AC) und der Kopf des NC repräsentieren das assoziative Kompartiment, sie sind mit präfrontalen kortikalen Arealen verknüpft. 
Die kortikostriatalen Projektionen der verschiedenen Schleifen unterscheiden sich u.a. in Bezug auf die Lateralität. Es konnte gezeigt werden, dass der SMC hauptsächlich ins ipsilaterale Striatum projiziert, während das SMA bilaterale Verknüpfungen ins Striatum hat dennoch bleibt der Großteil der Projektionen ipsilateral. Demgegenüber weist das Prä-SMA bilaterale Projektionen zum Striatum auf. Im Striatum selbst enden bilateral organisierte Afferenzen aus dem Kortex rostral von denjenigen, die unilateral organisiert sind. Es ist daher anzunehmen, dass die sensomotorische Schleife hauptsächlich eine unilaterale, die assoziative Schleife hingegen eine bilaterale Organisation aufweisen.

In-vivo-Bildgebung der kortikostriatalen Schleifen am Menschen ist u.a. mittels funktioneller Magnetresonanztomographie (fMRT) möglich. Es ist bereits gelungen, mittels verschiedener Aufgabenstellung die Schleifen spezifisch zu beeinflussen und somit voneinander zu differenzieren. So werden Aktivitäten in sensomotorischen Arealen durch Bewegungsamplitude und frequenz verändert. Hingegen zeigen Komplexität der Aufgabe, Selbst-Initiierung der Bewegung oder eigene Rhythmusvorgabe eine Beeinflussung von assoziativen Arealen. Die meisten bisherigen Studien beschränken sich auf die Untersuchung der dominanten rechten Hand und der kontralateralen Hirnhälfte.

In meiner Arbeit sollten die beiden motorischen Schleifen aufgrund ihrer Lateralitätseigenschaften unter Anwendung der fMRT identifiziert werden, d. h. aus einer Trennung der beidseitig von den nur einseitig aktivierten Arealen sollten die oben beschriebenen Schleifen erkennbar werden.

In einer Studie an 26 gesunden, rechtshändigen Jungen (mittleres Alter 11,8 Jahre, +/- 1,1 Jahre, Altersspanne 10,2-14,1 Jahre) erfolgte die fMRT-Untersuchung mit einem einfachen Studiendesign. Für beide Hände wurde ein robustes Fingerbewegungs-Paradigma als Blockdesign aufgesetzt. Um nicht nur rein exekutive, sondern auch assoziative Bewegungscharakteristika einzuschließen, führten die Probanden die Fingerbewegung in einem von ihnen selbst generierten Rhythmus durch.

Die resultierenden Aktivierungskarten ergaben das bekannte motorische Netzwerk aus primären und assoziativen Kortexanteilen, den Basalganglien, dem Thalamus und dem Kleinhirn. Durch eine Konjunktionsanalyse ermittlten wir Hirnregionen, die sowohl bei linkshändiger als auch bei rechtshändiger Fingerbewegung aktivieren. Es ergab sich ein bihemisphärielles, 
symmetrisches Muster aus rostralen Anteilen des SMA und des Putamens, der Inselrinde sowie des medialen Cerebellums. In einem nächsten Schritt führten wir eine Kontrastanalyse durch, wodurch seitenspezifisch reagierende Hirnareale zur Darstellung kamen. Diese waren der SMC, kaudale Anteile des SMA und des Putamens sowie der Thalamus, jeweils kontralateral zur bewegten Hand, außerdem ipsilaterale Anteile des Cerebellums.

Mit diesem einfachen und robusten Design gelang es, zwei kortiko-striato-thalamo-kortikale Schleifen mit ihren Lateralitätseigenschaften mittels fMRT zu visualisieren und zu differenzieren. Die lateralisierte sensomotorische Schleife umfasst den SMC, das SMAc, das kaudale Putamen, den Thalamus, sekundär sensorische Areale und das Kleinhirn. Ihre Projektionen verlaufen - mit Ausnahme cerebellärer Verbindungen - nur innerhalb der zur bewegten Körperseite kontralateralen Hemisphäre. Die assoziative Schleife hingegen ist bilateral organisiert, sie beinhaltet Prä-SMA, SMAr, das rostrale Putamen, die Insula und den cerebellären Vermis. Auch bei einseitiger Handbewegung werden Hirnregionen beider Hemisphären aktiviert.

Die in der Literatur postulierten kortiko-subkortikalen Schleifen konnten somit erstmals in vivo am Menschen dargestellt werden. Durch die Wahl der motorischen Aufgabe mit Beteiligung sowohl assoziativer als auch rein exekutiver, sensomotorischen Komponenten war es möglich, die verschiedenen Schleifen mit einem einzigen Paradigma zu erfassen. Die Untersuchung beider Hemisphären einerseits und sowohl kortikaler als auch subkortikaler Hirnregionen andererseits erlaubt das umfassende Studium der motorischen Netzwerke. Durch diese bisher unübliche Perspektive wird unsere Betrachtungsweise des motorischen Systems und seiner überaus komplexen Zusammenhänge erweitert.

Unser Studiendesign bietet einen neuen Ansatz zur Differenzierung der beiden Schleifen. Die im selbst generierten Rhythmus durchgeführte Bewegung des Zeigefingers der linken sowie der rechten Hand stellt eine einfache, elegante und reproduzierbare Aufgabe dar, die auch in Kontexten mit potentiell herausfordernden Probanden wie Kindern oder kognitiv eingeschränkten Patienten Einsatz finden kann.

Überdies schlagen wir ein modifiziertes Konzept für eine funktionelle Unterteilung des SMA vor. Demnach ist der rostrale Anteil des SMA bilateral organisiert und Teil einer funktionell assoziativen Schleife, während der kaudale Anteil eine klare Lateralisierung besitzt und der sensomotorischen Schleife zugehört. 
In der klinischen Neurologie sowohl des Kindes- als auch des Erwachsenenalters spielen Bewegungsstörungen eine große Rolle. Aufgrund der Komplexität des motorischen Systems kann bereits die Funktionsstörung einer einzelnen Hirnstruktur das sensible Gleichgewicht der Netzwerke stören.

Der Morbus Parkinson, die Chorea Huntington oder ein ischämischer Apoplex sind Beispiele für Erkrankungen, bei der es durch Funktionseinschränkung eines umschriebenen Hirnareals $\mathrm{zu}$ Alterationen in den motorischen Netzwerken und somit zu Bewegungsstörungen unterschiedlicher Art kommt. Insbesondere bei degenerativen Hirnerkrankungen ist der Verlauf stetig fortschreitend, es kommt zu erheblicher Einschränkung der Lebensqualität für die Patienten. Die Therapie umfasst medikamentöse, neurochirurgische und paramedizinische Maßnahmen. Insbesondere im Zeitalter der Entwicklung neuroprotektiver Verfahren nimmt die Früherkennung der Erkrankung einen zunehmend wichtigen Platz ein. Mithilfe der hier vorgestellten fMRT-Untersuchungen wäre bei Risiko- oder Verdachtspersonen möglicherweise schon zu einem noch symptomfreien Zeitpunkt die Erkennung gestörter motorischer Netzwerke und somit die Einleitung einer neuroprotektiven Therapie denkbar. Zur Beobachtung des Krankheitsverlaufs sowie zur Evaluation bereits bestehender Therapiekonzepte stellt das Verfahren eine ungefährliche und wiederholbare Methode dar.

In der Zusammenschau erweitern die gewonnenen Erkenntnisse meiner Arbeit unser Wissen über das motorische System und seine einzelnen Komponenten. Eine klinische Anwendung, z.B. im Bereich der Bewegungsstörungen, ist denkbar. Anhand der Krankheitsbilder des Morbus Parkinson, der Chorea Huntington, des ischämischen Apoplex oder des TouretteSyndroms wird beispielhaft deutlich, dass die funktionelle Bildgebung und der hier vorgestellte Ansatz zur verfeinerten Darstellung motorischer Netzwerke Beiträge zu Früherkennung und Diagnostik, zur Beobachtung eines Krankheitsverlaufs sowie zur Entwicklung und Evaluation neuer Therapiekonzepte leisten können. Auch für weitere wissenschaftliche Fragestellungen bietet sich der Ansatz aufgrund seiner verhältnismäßig einfachen Methodik und leichten Reproduzierbarkeit an. 


\section{Literaturverzeichnis}

Alexander GE, DeLong MR, Strick PL (1986): Parallel organization of functionally segregated circuits linking basal ganglia and cortex. Annu Rev Neurosci 9, 357-381

Alexander GE, Crutcher MD (1990): Functional architecture of basal ganglia circuits: neural substrates of parallel processing. Trends Neurosci $\underline{13}, 266-271$

Arthurs OJ, Boniface S (2002): How well do we understand the neural origins of the fMRI BOLD signal? Trends Neurosci $\underline{25}$, 27-31

Asanuma K, Ma Y, Huang C, Carbon-Correll M, Edwards C, Raymond D, Bressman SB, Moeller JR, Eidelberg D (2005): The metabolic pathology of dopa-responsive dystonia. Ann Neurol 57, 596-600

Asanuma K, Tang C, Ma Y, Dhawan V, Mattis P, Edwards C, Kaplitt MG, Feigin A, Eidelberg D (2006): Network modulation in the treatment of Parkinson's disease. Brain $\underline{129}, 2667-2678$

Boecker H, Dagher A, Ceballos-Baumann AO, Passingham RE, Samuel M, Friston KJ, Poline J, Dettmers C, Conrad B, Brooks DJ (1998): Role of the human rostral supplementary motor area and the basal ganglia in motor sequence control: investigations with $\mathrm{H} 2$ $15 \mathrm{O}$ PET. J Neurophysiol $\underline{79}$, 1070-1080

Boecker H, Jankowski J, Ditter P, Scheef L (2008): A role of the basal ganglia and midbrain nuclei for initiation of motor sequences. Neuroimage $\underline{39}, 1356-1369$

Buse J, August J, Bock N, Dörfel D, Rothenberger A, Roessner V (2012): Fine motor skills and interhemispheric transfer in treatment-naïve male children with Tourette syndrome. Dev Medicine Child Neuro, Onlinepublikation am 08.05.2012.

Deiber MP, Honda M, Ibanez V, Sadato N, Hallett M (1999): Mesial motor areas in selfinitiated versus externally triggered movements examined with fMRI: effect of movement type and rate. J Neurophysiol $\underline{81}$, 3065-3077

DeLong MR: The Basal Ganglia; in: Principles of Neural Science; hrsg v. Kandel ER, Schwartz JH, Jessell TM; 4. Auflage; McGraw-Hill Medical, New York, 2000, 853867

DeLong MR, Wichmann T (2007): Circuits and circuit disorders of the basal ganglia. Arch Neurol 64, 20-24

Di Martino A, Scheres A, Margulies DS, Kelly AM, Uddin LQ, Shehzad Z, Biswal B, Walters JR, Castellanos FX, Milham MP (2008): Functional connectivity of human striatum: a resting state FMRI study. Cereb Cortex $\underline{18}$, 2735-2747

Feigin A, Leenders KL, Moeller JR, Missimer J, Kuenig G, Spetsieris P, Antonini A, Eidelberg D (2001): Metabolic network abnormalities in early Huntington's disease: an [(18)F]FDG PET study. J Nucl Med $\underline{42}, 1591-1595$ 
Feigin A, Tang C, Ma Y, Mattis P, Zgaljardic D, Guttman M, Paulsen JS, Dhawan V, Eidelberg D (2007): Thalamic metabolism and symptom onset in preclinical Huntington's disease. Brain $\underline{130}, 2858-2867$

Flaherty AW, Graybiel AM (1993): Two input systems for body representations in the primate striatal matrix: experimental evidence in the squirrel monkey. J Neurosci $\underline{13}$, $1120-1137$

Forman SD, Cohen JD, Fitzgerald M, Eddy WF, Mintun MA, Noll DC (1995): Improved assessment of significant activation in functional magnetic resonance imaging (fMRI): use of a cluster-size threshold. Magn Reson Med 33, 636-647

Fox PT, Raichle ME (1986): Focal physiological uncoupling of cerebral blood flow and oxidative metabolism during somatosensory stimulation in human subjects. Proc Natl Acad Sci U S A $\underline{83}, 1140-1144$

Frahm J, Haase A, Matthaei D (1986a): Rapid NMR imaging of dynamic processes using the FLASH technique. Magn Reson Med $\underline{3}, 321-327$

Frahm J, Haase A, Matthaei D (1986b): Rapid three-dimensional MR imaging using the FLASH technique. J Comput Assist Tomogr 10, 363-368

Gerardin E, Lehericy S, Pochon JB, Tezenas du Montcel S, Mangin JF, Poupon F, Agid Y, Le Bihan D, Marsault C (2003): Foot, hand, face and eye representation in the human striatum. Cereb Cortex $\underline{13}, 162-169$

Giedd JN, Lalonde FM, Celano MJ, White SL, Wallace GL, Lee NR, Lenroot RK (2009): Anatomical brain magnetic resonance imaging of typically developing children and adolescents. J Am Acad Child Adolesc Psychiatry 48, 465-470

Goebel R, Kriegeskorte N: Funktionelle Magnetresonanztomographie. 1. Auflage; Schattauer $\mathrm{GmbH}$, Stuttgart 2005

Grafton ST, Arbib MA, Fadiga L, Rizzolatti G (1996): Localization of grasp representations in humans by positron emission tomography. 2. Observation compared with imagination. Exp Brain Res 112, 103-111

Haaland KY, Elsinger CL, Mayer AR, Durgerian S, Rao SM (2004): Motor sequence complexity and performing hand produce differential patterns of hemispheric lateralization. J Cogn Neurosci 16, 621-636

Jankowski J, Scheef L, Huppe C, Boecker H (2009): Distinct striatal regions for planning and executing novel and automated movement sequences. Neuroimage 44, 1369-1379

Jenkins IH, Passingham RE, Brooks DJ (1997): The effect of movement frequency on cerebral activation: a positron emission tomography study. J Neurol Sci 151, 195-205

Jueptner M, Weiller C (1998): A review of differences between basal ganglia and cerebellar control of movements as revealed by functional imaging studies. Brain 121 ( $\mathrm{Pt} \mathrm{8}$ ), $1437-1449$ 
Kemp JM, Powell TP (1971): The connexions of the striatum and globus pallidus: synthesis and speculation. Philos Trans R Soc Lond B Biol Sci 262, 441-457

Kloppel S, Henley SM, Hobbs NZ, Wolf RC, Kassubek J, Tabrizi SJ, Frackowiak RS (2009): Magnetic resonance imaging of Huntington's disease: preparing for clinical trials. Neuroscience $164,205-219$

Kraft E, Chen AW, Flaherty AW, Blood AJ, Kwong KK, Jenkins BG (2007): The role of the basal ganglia in bimanual coordination. Brain Res $\underline{1151}, 62-73$

Kunzle H (1975): Bilateral projections from precentral motor cortex to the putamen and other parts of the basal ganglia. An autoradiographic study in Macaca fascicularis. Brain Res $\underline{88}, 195-209$

Lauterbur P (1973): Image formation by induced local interactions: Examples employing nuclear magnetic resonance. Nature 242, 190-191

Lawrence AD, Sahakian BJ, Robbins TW (1998): Cognitive functions and corticostriatal circuits: insights from Huntington's disease. Trends Cogn Sci 2, 379-388

Leh SE, Ptito A, Chakravarty MM, Strafella AP (2007): Fronto-striatal connections in the human brain: a probabilistic diffusion tractography study. Neurosci Lett $\underline{419}, 113-118$

Lehericy S, van de Moortele PF, Lobel E, Paradis AL, Vidailhet M, Frouin V, Neveu P, Agid Y, Marsault C, Le Bihan D (1998): Somatotopical organization of striatal activation during finger and toe movement: a 3-T functional magnetic resonance imaging study. Ann Neurol 44, 398-404

Lehericy S, Ducros M, Krainik A, Francois C, Van de Moortele PF, Ugurbil K, Kim DS (2004a): 3-D diffusion tensor axonal tracking shows distinct SMA and pre-SMA projections to the human striatum. Cereb Cortex 14, 1302-1309

Lehericy S, Ducros M, Van de Moortele PF, Francois C, Thivard L, Poupon C, Swindale N, Ugurbil K, Kim DS (2004b): Diffusion tensor fiber tracking shows distinct corticostriatal circuits in humans. Ann Neurol 55, 522-529

Lehericy S, Gerardin E, Delmaire C (2006): [Neuroimaging of subcortical circuits]. Encephale 32, S25-30

Lenroot RK, Giedd JN (2006): Brain development in children and adolescents: insights from anatomical magnetic resonance imaging. Neurosci Biobehav Rev $\underline{30}$, 718-729

Nakano K, Kayahara T, Tsutsumi T, Ushiro H (2000): Neural circuits and functional organization of the striatum. J Neurol 247 Suppl 5, V1-15

Nandhagopal R, McKeown MJ, Stoessl AJ (2008): Functional imaging in Parkinson disease. Neurology $\underline{70}, 1478-1488$

Ogawa S, Lee TM, Kay AR, Tank DW (1990): Brain magnetic resonance imaging with contrast dependent on blood oxygenation. Proc Natl Acad Sci U S A $\underline{87}, 9868-9872$ 
Oldfield RC (1971): The assessment and analysis of handedness: the Edinburgh inventory. Neuropsychologia $\underline{9}, 97-113$

Parent A (1990): Extrinsic connections of the basal ganglia. Trends Neurosci 13, 254-258

Parent A, Hazrati LN (1995): Functional anatomy of the basal ganglia. I. The cortico-basal ganglia-thalamo-cortical loop. Brain Res Brain Res Rev 20, 91-127

Pauling L (1935): The Oxygen Equilibrium of Hemoglobin and Its Structural Interpretation. Proc Natl Acad Sci U S A 21, 186-191

Paulsen JS (2009): Functional imaging in Huntington's disease. Exp Neurol 216, 272-277

Paus T, Collins DL, Evans AC, Leonard G, Pike B, Zijdenbos A (2001): Maturation of white matter in the human brain: a review of magnetic resonance studies. Brain Res Bull $\underline{54}$, 255-266

Picard N, Strick PL (1996): Motor areas of the medial wall: a review of their location and functional activation. Cereb Cortex $\underline{6}, 342-353$

Picard N, Strick PL (2001): Imaging the premotor areas. Curr Opin Neurobiol 11, 663-672

Politis M (2011): Optimizing functional imaging protocols for assessing the outcome of fetal cell transplantation in Parkinson's disease. BMC Med $\underline{9}, 50$

Postuma RB, Dagher A (2006): Basal ganglia functional connectivity based on a metaanalysis of 126 positron emission tomography and functional magnetic resonance imaging publications. Cereb Cortex $\underline{16}, 1508-1521$

Pourfar M, Feigin A, Tang CC, Carbon-Correll M, Bussa M, Budman C, Dhawan V, Eidelberg D Abnormal metabolic brain networks in Tourette syndrome. Neurology $\underline{76}$, 944-952

Roessner V, Overlack S, Schmidt-Samoa C, Baudewig J, Dechent P, Rothenberger A, Helms G (2010): Increased putamen and callosal motor subregion in treatment-naive boys with Tourette syndrome indicates changes in the bihemispheric motor network. J Child Psychol Psychiatry 52, 306-314

Roessner V, Wittfoth M, Schmidt-Samoa C, Rothenberger A, Dechent P, Baudewig J (2011): Altered motor network recruitment during finger tapping in boys with Tourette syndrome. Hum Brain Mapp 33, 666-675

Roessner V, Wittfoth M, August JM, Rothenberger A, Baudewig J, Dechent P (im Druck): Finger tapping-related activation differences in treatment-naive pediatric Tourettesyndrome: a comparison of the preferred and non-preferred hand. J Child Psychol Psychiatry.

Roth M, Decety J, Raybaudi M, Massarelli R, Delon-Martin C, Segebarth C, Morand S, Gemignani A, Decorps M, Jeannerod M (1996): Possible involvement of primary mo- 
tor cortex in mentally simulated movement: a functional magnetic resonance imaging study. Neuroreport $\underline{7}, 1280-1284$

Sadato N, Campbell G, Ibanez V, Deiber M, Hallett M (1996a): Complexity affects regional cerebral blood flow change during sequential finger movements. J Neurosci $\underline{16}$, 26912700

Sadato N, Ibanez V, Deiber MP, Campbell G, Leonardo M, Hallett M (1996b): Frequencydependent changes of regional cerebral blood flow during finger movements. J Cereb Blood Flow Metab 16, 23-33

Selemon LD, Goldman-Rakic PS (1985): Longitudinal topography and interdigitation of corticostriatal projections in the rhesus monkey. J Neurosci $\underline{5}$, 776-794

Stephan KM, Fink GR, Passingham RE, Silbersweig D, Ceballos-Baumann AO, Frith CD, Frackowiak RS (1995): Functional anatomy of the mental representation of upper extremity movements in healthy subjects. J Neurophysiol $\underline{73}$, 373-386

Stoessl AJ (2011): Neuroimaging in Parkinson's disease. Neurotherapeutics $\underline{8}, 72-81$

Talairach J, Tournoux P: Co-planar stereotaxic atlas of the human brain: 3-dimensional proportional system: an approach to cerebral imaging. Thieme Medical Publishers, New York 1988

Tang CC, Poston KL, Dhawan V, Eidelberg D (2010): Abnormalities in metabolic network activity precede the onset of motor symptoms in Parkinson's disease. J Neurosci $\underline{30}$, 1049-1056

Thulborn KR, Waterton JC, Matthews PM, Radda GK (1982): Oxygenation dependence of the transverse relaxation time of water protons in whole blood at high field. Biochim Biophys Acta $\underline{714}$, 265-270

Toga AW, Thompson PM, Sowell ER (2006): Mapping brain maturation. Trends Neurosci $\underline{29}, 148-159$

Trepel M: Neuroanatomie. Struktur und Funktion. 3. Auflage; Urban und Fischer, München 2004

Tyszka JM, Grafton ST, Chew W, Woods RP, Colletti PM (1994): Parceling of mesial frontal motor areas during ideation and movement using functional magnetic resonance imaging at 1.5 tesla. Ann Neurol $\underline{35}, 746-749$

Vorobiev V, Govoni P, Rizzolatti G, Matelli M, Luppino G (1998): Parcellation of human mesial area 6: cytoarchitectonic evidence for three separate areas. Eur J Neurosci 10, 2199-2203

Ward NS (2006): The neural substrates of motor recovery after focal damage to the central nervous system. Arch Phys Med Rehabil 구, S30-35

Weir DW, Sturrock A, Leavitt BR (2011): Development of biomarkers for Huntington's disease. Lancet Neurol 10, 573-590 
Weltärztebund (2001): World Medical Association Declaration of Helsinki. Ethical principles for medical research involving human subjects. Bull World Health Organ $\underline{79}$, 373-374

Wiesendanger E, Clarke S, Kraftsik R, Tardif E (2004): Topography of cortico-striatal connections in man: anatomical evidence for parallel organization. Eur J Neurosci $\underline{20}$, 1915-1922

Wiesendanger M, Rouiller EM, Kazennikov O, Perrig S (1996): Is the supplementary motor area a bilaterally organized system? Adv Neurol $\underline{70}$, 85-93

Zilles K, Schlaug G, Geyer S, Luppino G, Matelli M, Qu M, Schleicher A, Schormann T (1996): Anatomy and transmitter receptors of the supplementary motor areas in the human and nonhuman primate brain. Adv Neurol 70, 29-43 


\section{Abbildungsverzeichnis}

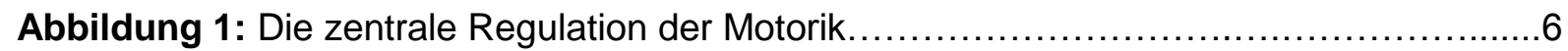

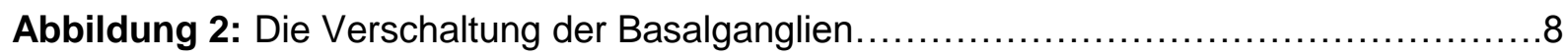

Abbildung 3: Verschiedene Modelle kortiko-basalganglien-thalamo-kortikaler Schleifen.......9

Abbildung 4: Die Entstehung des BOLD-Signals ....................................... 15

Abbildung 5: Charakteristischer Zeitverlauf des fMRT-Signals .........................16

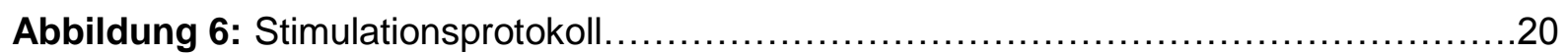

Abbildung 7: Das Allgemeine Lineare Modell in einem vereinfachten Schema..............23

Abbildung 8: Haupteffekte. Gruppenanalyse nach dem Random-Effects-Modell............28

Abbildung 9: Konjunktionsanalyse; Gruppenanalyse nach dem Random-Effects-Modell...30

Abbildung 10: Die rostrale SMA in der Konjunktionsanalyse mit Signal-Zeit-Verlauf........31

Abbildung 11: Die Putamina in der Konjunktionsanalyse mit Signal-Zeit-Verläufen..........31

Abbildung 12: Kontrastanalyse; Gruppenanalyse nach dem Random-Effects-Modell.......33

Abbildung 13: Der Primär Sensomotorische Kortex beidseits in der Kontrastanalyse........34

Abbildung 14: Die kaudalen Putamina und die Thalami beidseits in der Kontrastanalyse...34

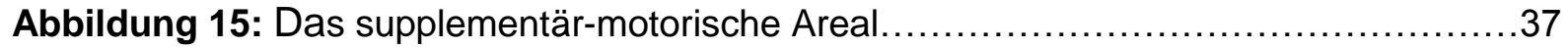

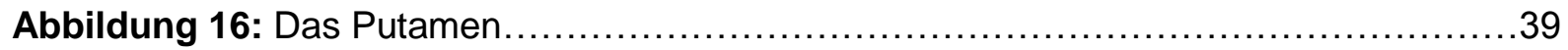

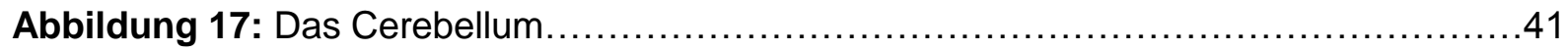

Abbildung 18: Modell für kortikostriatale Projektionen..................................43

Abbildung 19: Die beim Morbus Parkinson charakteristischen Veränderungen..............45

Abbildung 20: Patient mit einem mittelschwer ausgeprägten Morbus Parkinson............46

Abbildung 21: Die beim Morbus Huntington charakteristischen Veränderungen.............48

Abbildung 22: Aktivierungskarten eines Schlaganfall-Patienten ........................50

Abbildung 23: Vergleich einer Gruppe von TS-Patienten mit gesunden Kontrollprobanden53 


\section{Tabellenverzeichnis}

Tabelle 1: Haupteffekte; Gruppenanalyse nach dem Random-effects-Modell

Tabelle 2: Konjunktionsanalyse; Gruppenanalyse nach dem Random-Effects-Modell.......29

Tabelle 3: Gruppenanalyse; Kontrasteffekte nach dem Random-Effects-Modell 32 


\section{Danksagung}

Ich bedanke mich in erster Linie herzlich bei Herrn PD Dr. Peter Dechent für seine geduldige und immer wieder motivierende Betreuung. Weiter möchte ich Herrn Prof. Dr. Veit Rössner sowie Herrn Dr. Jürgen Baudewig für ihre Anleitung, Einarbeitung und Begleitung danken. Frau Ilona Pfahlert gebührt Dank für die Durchführung der MRT-Messungen.

Nicht zuletzt möchte ich mich ausdrücklich bei den Probanden und ihren Eltern für ihr Mitwirken in unserem Projekt bedanken. 


\section{Lebenslauf von Julia Margarethe August geb. Böhm}

Am 01.01.1982 wurde ich als erstes von vier Kindern meiner Eltern in Hannover geboren. Von 1988 bis 1986 besuchte ich die Grundschule Bonner Straße, anschließend bis 1994 die Orientierungsstufe Altenbekener Damm in Hannover. Meine Gymnasialzeit absolvierte ich an der Elsa-Brändström-Schule Hannover bis zum Abitur im Juni 2001.

Im Wintersemester 2001/02 begann ich das Studium der Psychologie an der Georg-AugustUniversität Göttingen. Nach einem Semester wechselte ich das Studienfach und begann im Sommersemester 2002 das Studium der Humanmedizin in Göttingen. Pflegepraktika absolvierte ich in Hamburg und Molde, Norwegen. Die 1. Ärztliche Prüfung legte ich mit der Note 2,33 (gut) ab. Im klinischen Studienabschnitt erhielt ich über ein ERASMUS-Stipendium die Möglichkeit zu einem Auslandsstudienjahr in Lyon, Frankreich; dort verbrachte ich das Wintersemester 2005/06 und das Sommersemester 2006. Famulaturen absolvierte ich in den Fächern Anästhesie, Neuropädiatrie, Innere Medizin und Kinder- und Jugendpsychiatrie.

Das Praktische Jahr begann ich im August 2007 mit meinem Wahlfach Pädiatrie im Klinikum Wolfsburg. Ab Dezember 2007 schloss sich das Tertial in der Inneren Medizin des St. Martini-Krankenhauses Duderstadt an. Das Tertial in der Chirurige absolvierte ich im Krankenhaus Molde, Norwegen, von März bis Juli 2008.

Die 2. Ärztliche Prüfung legte ich im November 2008 mit der Note 1,5 (sehr gut) ab.

Seit dem 01.03.2009 bin ich als Assistenzärztin in der Abteilung Pädiatrie II der Universitätsmedizin Göttingen beschäftigt. 